\title{
INVESTIGATING THE FRACTURE RESISTANCE OF BIOACTIVE GLASS COATINGS ON METALLIC IMPLANTS \\ by
}

Ali (Mike) Matinmanesh

BSc, Mechanical Engineering, Iran University of Science and Technology, 2010

MSc, Mechanical Engineering, Politecnico di Milano, 2013

\author{
A dissertation \\ presented to Ryerson University \\ in partial fulfillment of the \\ requirements for the degree of \\ Doctor of Philosophy \\ in the Program of \\ Mechanical and Industrial Engineering
}

Toronto, Ontario, Canada, 2018

(C) Ali (Mike) Matinmanesh 2018 


\section{Author's Declaration}

\section{AUTHOR'S DECLARATION FOR ELECTRONIC SUBMISSION OF A DISSERTATION}

I hereby declare that I am the sole author of this dissertation. This is a true copy of the dissertation, including any required final revisions, as accepted by my examiners.

I authorize Ryerson University to lend this dissertation to other institutions or individuals for the purpose of scholarly research.

I further authorize Ryerson University to reproduce this dissertation by photocopying or by other means, in total or in part, at the request of other institutions or individuals for the purpose of scholarly research.

I understand that my dissertation may be made electronically available to the public. 


\section{Abstract}

Investigating the Fracture Resistance of Bioactive Glass Coatings on Metallic Implants

Doctor of Philosophy, 2018

Ali (Mike) Matinmanesh

Mechanical \& Industrial Engineering,

Ryerson University

Bioactive glasses have been used experimentally as coatings for medical implants because of their good osseointegration properties and ability to inhibit bacterial proliferation. However, the available literature lacks quantitative studies for characterizing their mechanical properties. This research postulates two fracture mechanics testing methodologies that facilitate measuring the nearly pure mode I (opening) and mode II (shearing) critical strain energy release rate ( $\left.G_{I C}, G_{I I C}\right)$ of the coating/substrate system. Using these methodologies, the effects of coating thickness, glass composition and degradation on the $G_{I C}$ and $G_{I I C}$ of the system were evaluated. The developed mode I testing methodology was applied on a silicate bioactive glass/Ti6Al4V substrate system and it was found that increasing the coating thickness from 90 to $390 \mu \mathrm{m}$, decreased the measured $G_{I C}$ of the system significantly, from 6.2 to $2.5 \mathrm{~J} / \mathrm{m}^{2}$. This decrease was found to be due to the increase in the residual stresses in the thicker coatings. The mode I testing methodology was then applied on two series of silica-based and borate-based glass coating, with increasing amounts of $\mathrm{TiO}_{2}$ incorporated, and it was observed that an increase in the content of $\mathrm{TiO}_{2}$ in the glasses resulted in an increase in the $G_{I C}$ for both the bulk glass and for the coating/substrate system. The borate-based series was found to have a closer CTE to the substrate compared to the silica counterpart, suggesting that use of such glasses as coatings can minimize the chances of delamination and cracking. Incorporating $\mathrm{SrCO}_{3}$ in a series of borate bioactive glass coating also proved to significantly increase the $G_{I C}$ and $G_{I I C}$ of the system. In order to study the effect of 
degradation, the borate bioactive glass coatings on Ti6Al4V substrates were immersed in deionized water for different time periods, dried and tested. It was found that after $17 \%$ weight loss of the glass, the $G_{I C}$ and $G_{I I C}$ of the coating/substrate system for all compositions decreased by at least $80 \%$. 


\section{Acknowledgements}

I would like to thank my supervisors, Dr. Marcello Papini and Dr. Mark Towler, for their guidance and encouragement, and the opportunity to work on this amazing project. I would like to also acknowledge the financial support of the Canadian Institute of Health Research (CIHR) and the Natural Sciences and Engineering Research Council of Canada (NSERC) under the Collaborative Health Research Program (CHRP; contract \# 315694-DAN).

This work would not have been completed without the help of Ryerson's lab technicians, Chao Ma, Alan Machin, Qiang Li and Roy Churaman. The technical support of Dr. Declan Curran, and Dr, Jan Spelt was also crucial.

I would like to also thank my incredible lab mates, Omar, Li, Adel, Alireza, Basel, Saad, Faizan, Romina, Leyla, Saidur and Andrew for making such a wonderful research team, and my best buddies, Omid and Ramin (in alphabetical order) for 12 years of brotherhood. Moreover, the assistance of Dr. Owen Clarkin, Dr. Ali Nouhi, Sunjeev Phull, Isaac Beniluz and Bharath Krishnan in performing the experiments and data collection is greatly appreciated.

Last but not least, I would like to thank my brother, Dylan, for giving me directions my entire life and being the best big brother I could ever ask for, and my amazing girlfriend, Nikita, for brightening up my days and bringing endless love and joy into my life. To conclude, I would like to dedicate this thesis to my parents, the best gifts I have ever been given, for all of their sacrifices without which I could never be here. 


\section{Table of Contents}

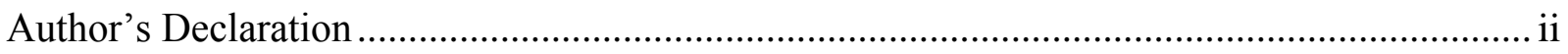

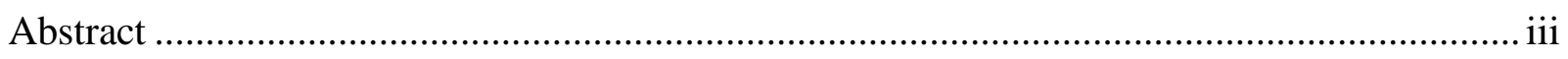

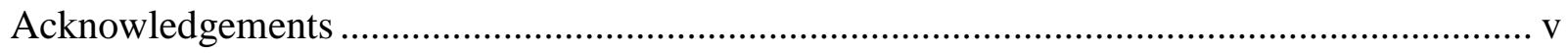

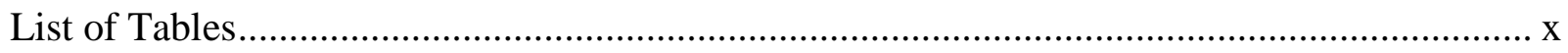

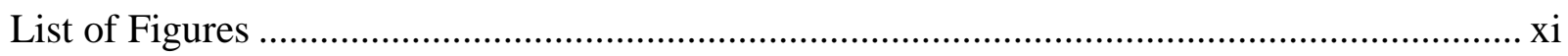

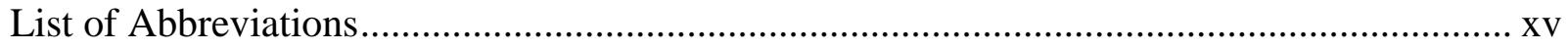

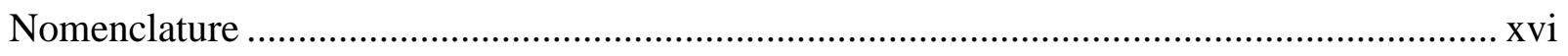

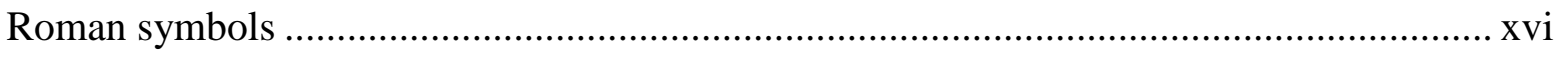

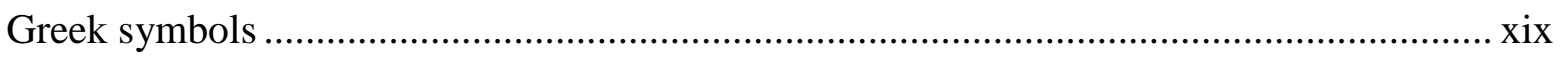

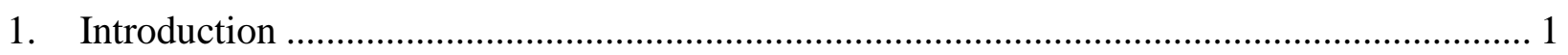

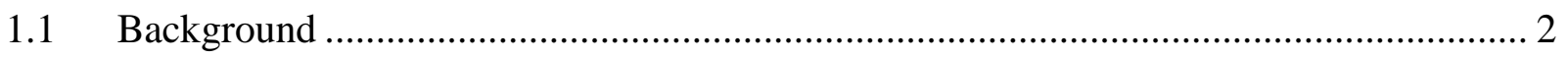

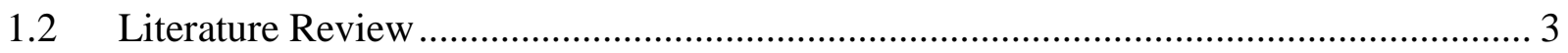

1.2.1 Coating metallic implants with glass ....................................................... 3

1.2.2 Adhesion between bioactive glass coating and Ti6Al4V .................................... 4

1.2.3 Measurement of coating adhesion ........................................................... 5

1.2.4 Effect of coating thickness on adhesion.................................................. 14

1.2.5 Effect of glass composition on adhesion ............................................... 14

1.2.6 Effect of degradation of the bioactive glass on adhesion .................................. 15

1.2.7 Effect of substrate roughness on adhesion ................................................. 17

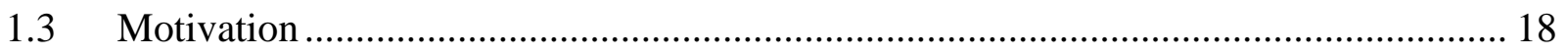

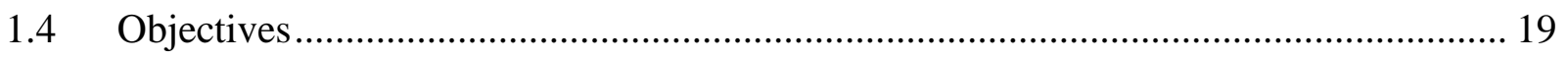

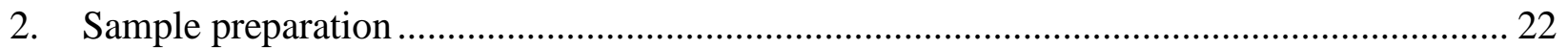




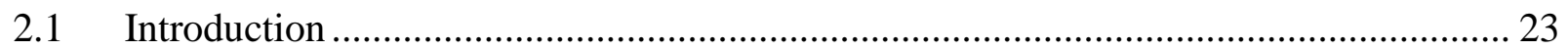

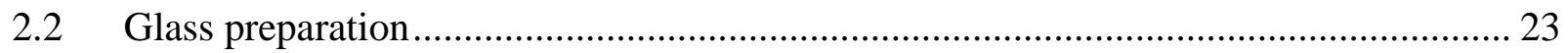

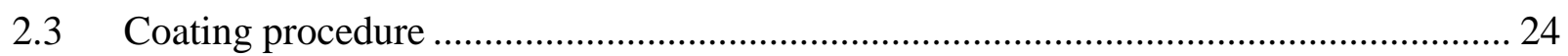

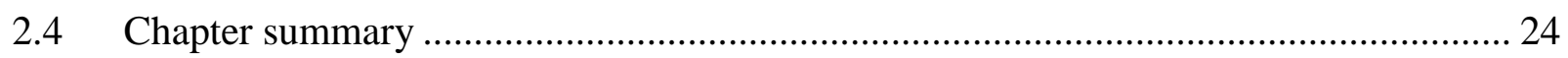

3. Quantitative evaluation of the mode I critical strain energy release rate .............................. 25

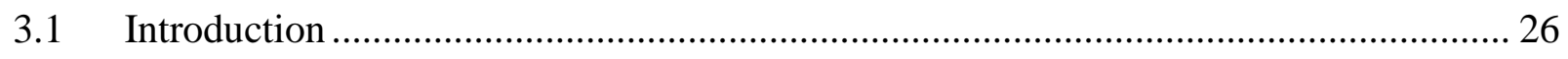

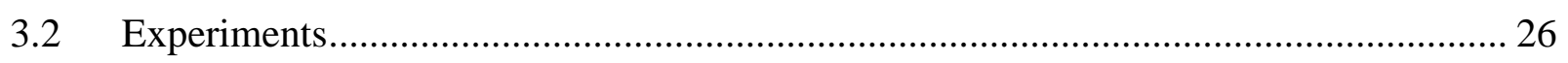

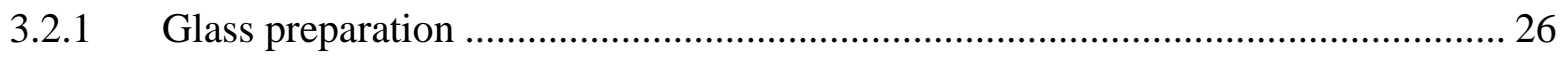

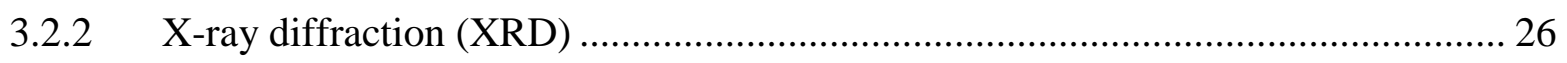

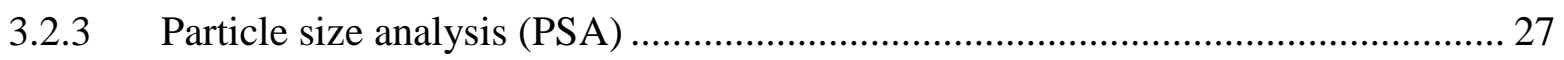

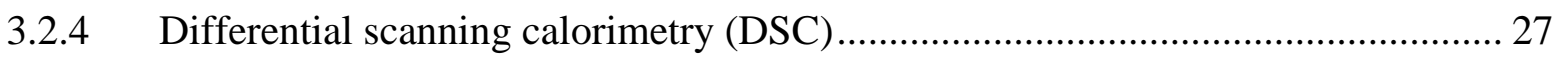

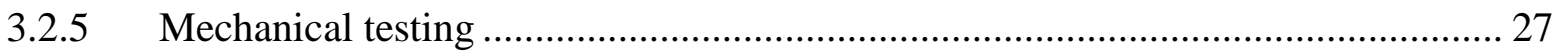

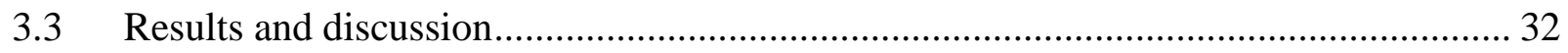

3.3.1 X-ray diffraction (XRD) ……………………................................................. 32

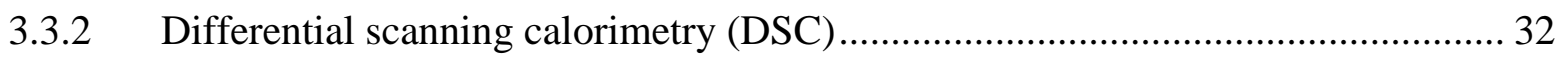

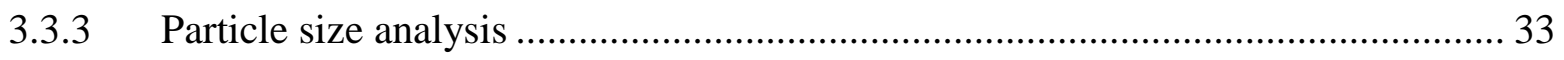

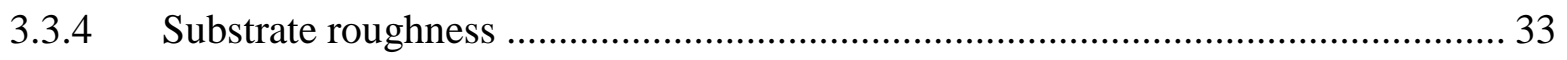

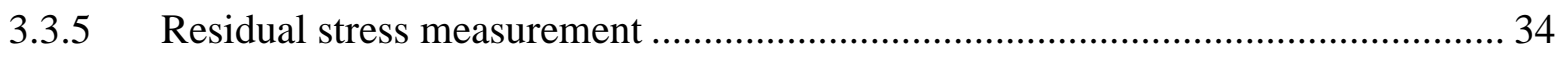

3.3.6 Mode I critical strain energy release rate _........................................................... 34

3.3.7 Crack path and mode ratio ……………………............................................... 36

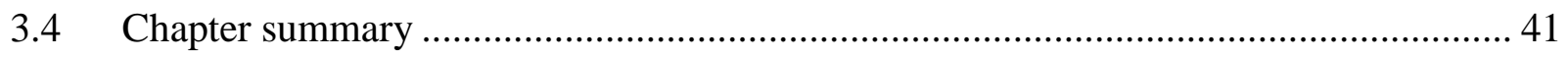

4. Silica-Based and Borate-Based, Titania-Containing Bioactive Coatings Characterization .. 43

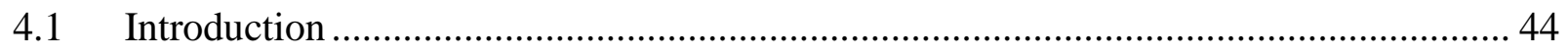

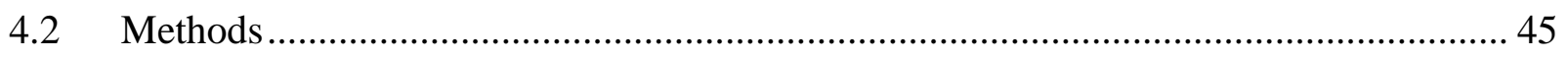

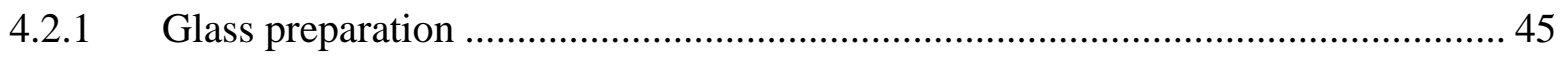




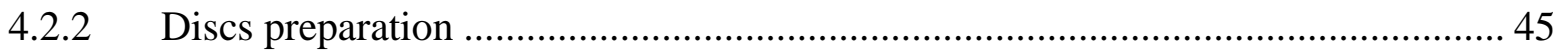

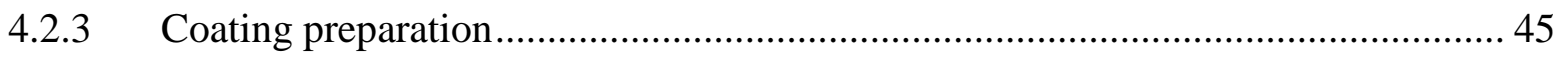

4.2.4 Coefficient of thermal expansion (CTE) measurement by linear dilatometry........ 45

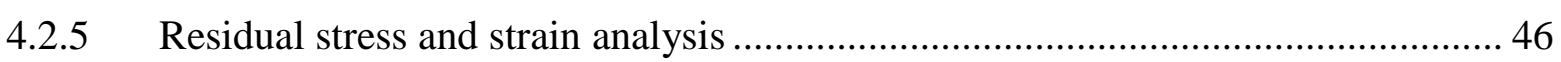

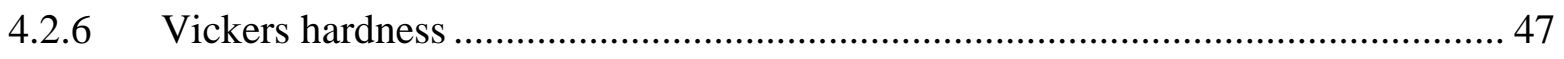

4.2.7 Mode I critical strain energy release rate of the bulk glass.................................... 47

4.2.8 Coating/substrate system mode I strain energy release rate .................................. 48

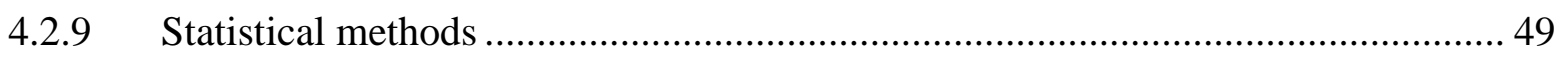

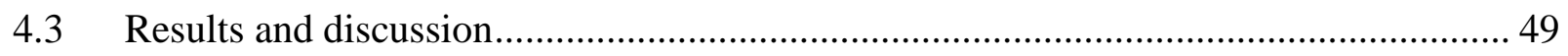

4.3.1 Coefficient of thermal expansion (CTE) measurement by linear dilatometry........ 49

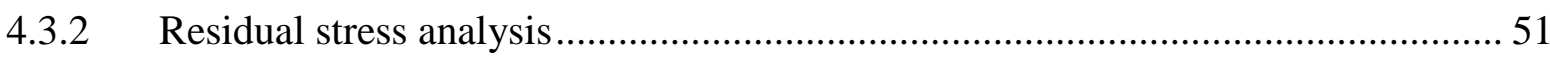

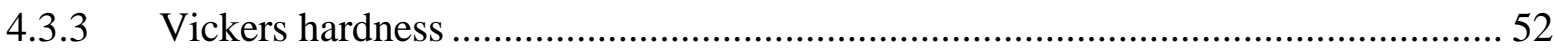

4.3.4 Mode I critical strain energy release rate of bulk glass using Vickers indentation 53

4.3.5 Mode I critical strain energy release rate of the coating/substrate system .............. 55

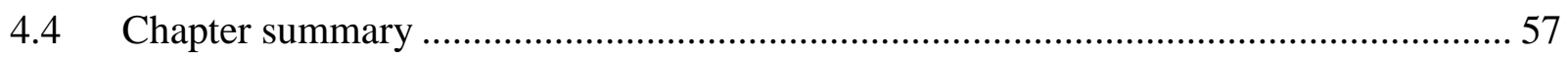

5. Quantifying the mode II critical strain energy release rate .................................................. 58

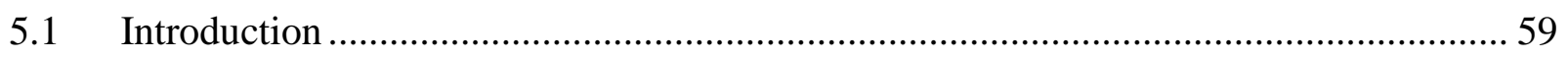

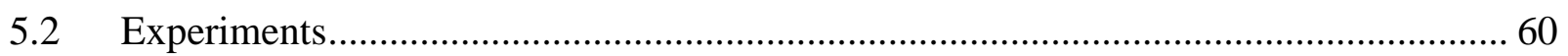

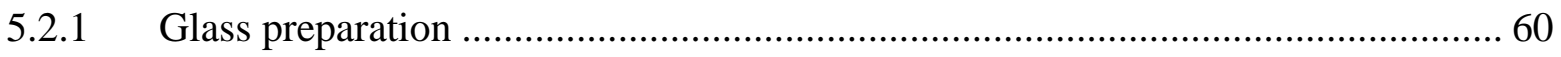

5.2.2 Measurement of coefficient of thermal expansion.............................................. 60

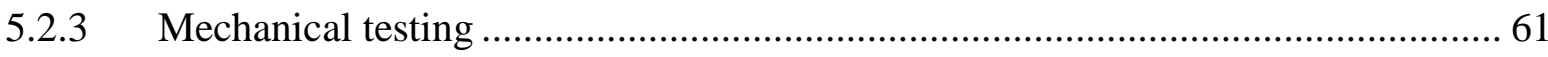

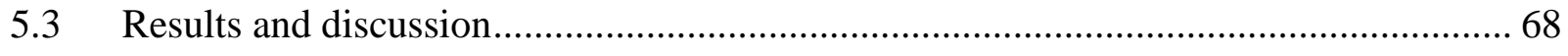

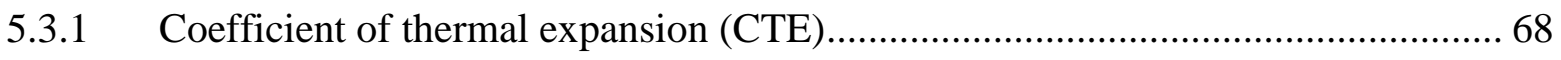

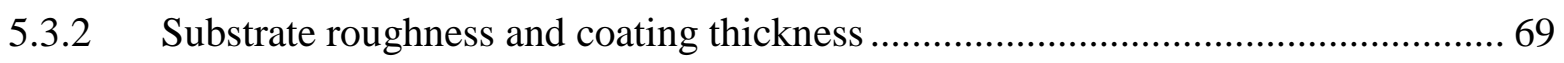

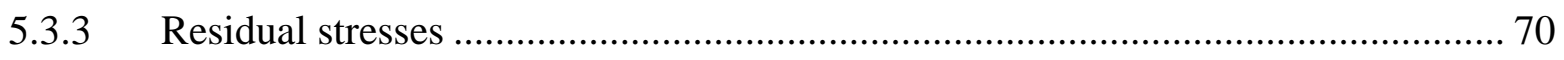


5.3.4 Mode II critical strain energy release rate................................................ 72

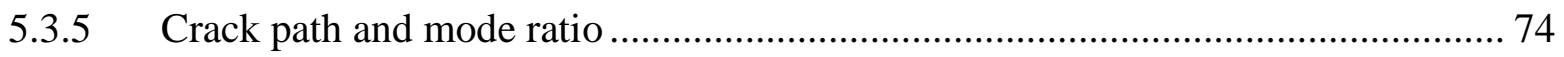

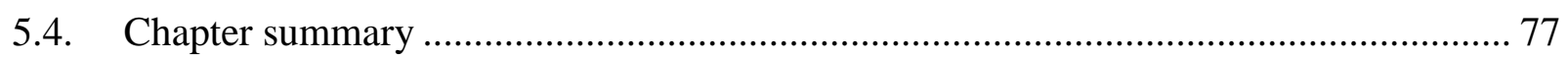

6. Evaluating the critical strain energy release rate after degradation................................... 79

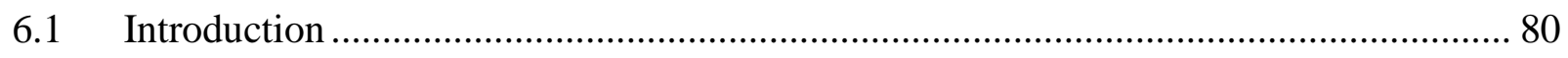

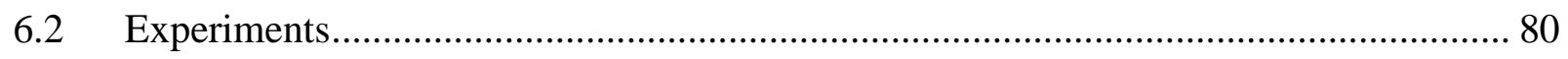

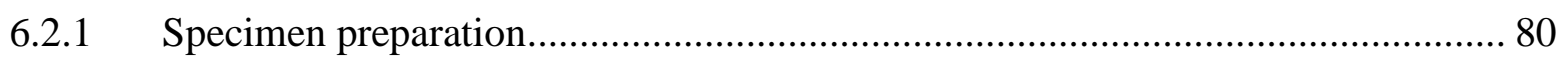

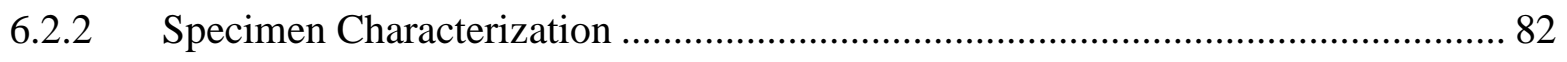

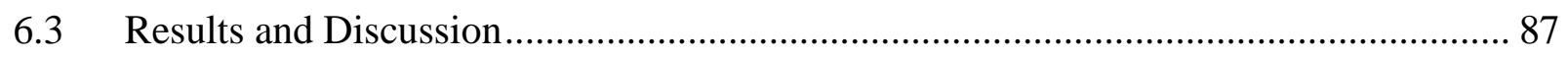

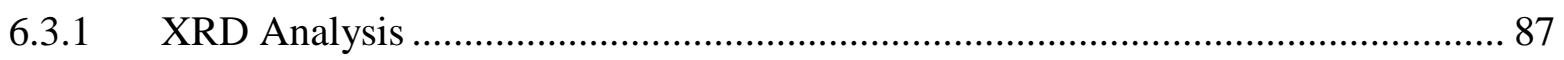

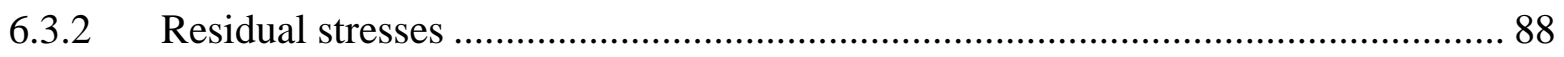

6.3.3 Weight loss due to degradation ................................................................ 88

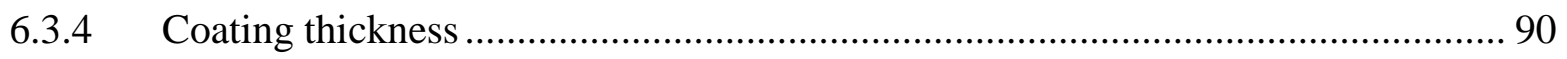

6.3.5 Critical Mode I and Mode II strain energy release rate ................................... 92

6.3.6 Effect of roughness on $G_{I C}$ and $G_{I I C}$ of the degraded samples........................... 96

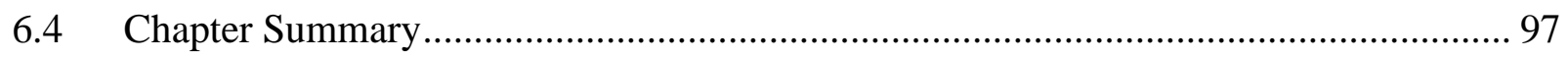

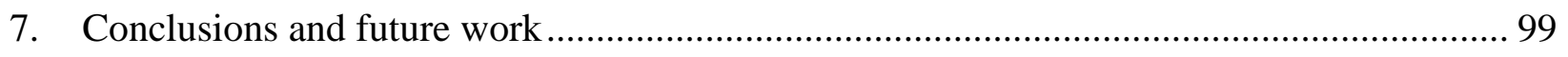

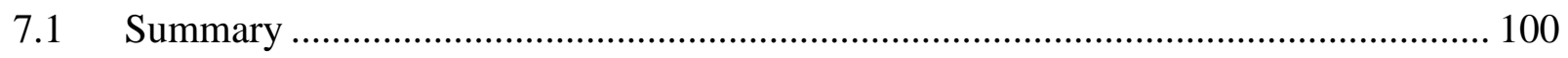

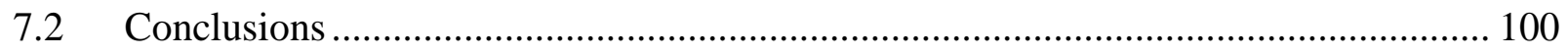

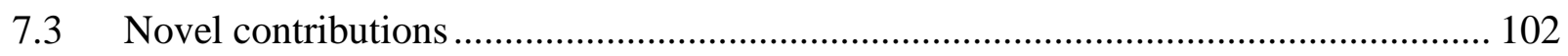

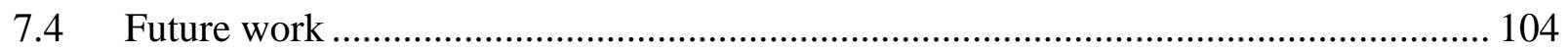

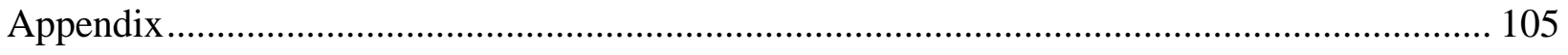

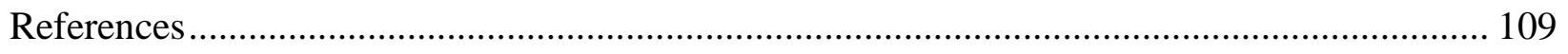




\section{List of Tables}

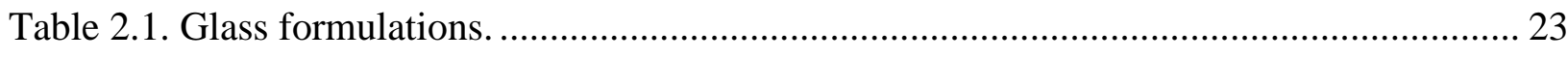

Table 2.2. Glass transition, crystallization and coating temperatures for different glass

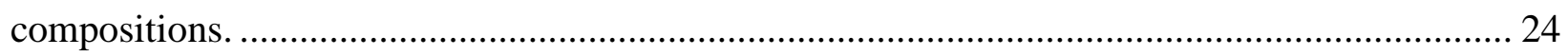

Table 3.1. Coating thickness $t_{c}$, signed curvature $k$, residual stresses $\boldsymbol{\sigma c i n}$, and residual coating

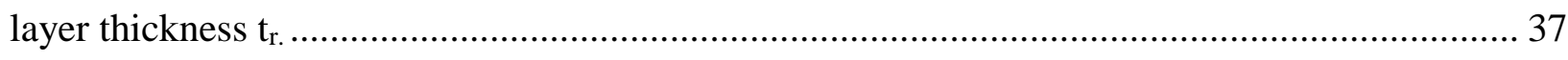

Table 5.1. Coating thickness $t_{c}$, signed curvature $k$, residual stresses $\boldsymbol{\sigma} \boldsymbol{c}$ and residual coating layer thickness $t_{r}$ for the mode II specimens. The residual stresses are calculated from the deformed substrate profiles as explained in Section 5.2.3.2 …............................................ 69 


\section{List of Figures}

Figure 1.1. Use of Ti6Al4V orthopedic implants in hip replacement surgery (a) ${ }^{7}$. Hydroxyapatite (HA) is currently used as coating for these implants ${ }^{8}$.

Figure 1.2. Adhesion scratch test ${ }^{52}$ quantifies the adhesion between the coating and substrate by measuring the critical normal force under which a moving diamond stylus creates a well-defined

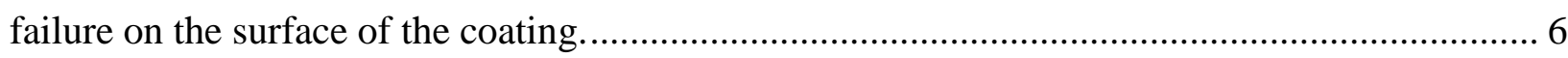

Figure 1.3. Qualitative indentation test ${ }^{10}$ characterizes the adhesion as strong if the crack emanated from an interfacial indent grows in the coating rather than the coating/substrate

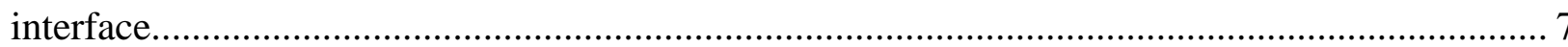

Figure 1.4. Vickers indentation fracture test ${ }^{70}$ measures the fracture toughness from direct measurements of cracking in an indentation test. ............................................................ 10

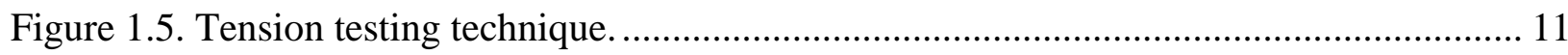

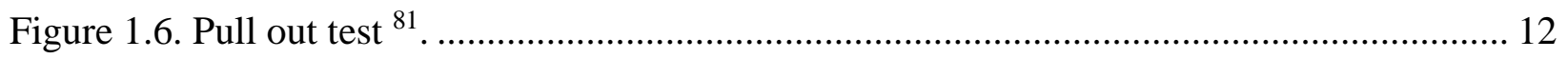

Figure 1.7. Double Cantilever Beam (DCB) Specimen for mode I (a), mode II (b) and mixed

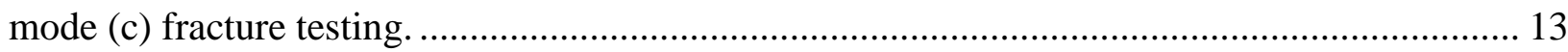

Figure 3.1. Double layer DCB specimen. Drawing is not to scale. ....................................... 30

Figure 3.2. XRD pattern for SRT0. + indicates Sodium Calcium Phosphate Silicate

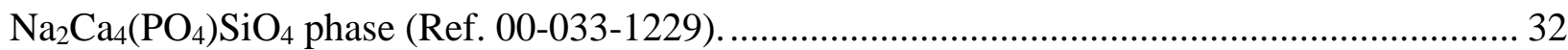

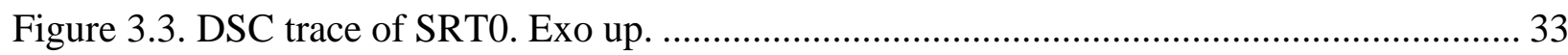

Figure 3.4. Typical force vs. crosshead displacement and force versus normalized crack length curves obtained from DCB tests. The dashed line is to help guide the eye.

Figure 3.5. Critical strain energy release rate as function of normalized crack length for specimens having coating thickness (+/- standard deviation) of (a) $90 \pm 12 \mu \mathrm{m}$ (b) $250 \pm 22 \mu \mathrm{m}$ and (c) $390 \pm 32 \mu \mathrm{m}$. The mean values of GIC, shown with horizontal lines, were $6.2 \mathrm{~J} / \mathrm{m} 2,4.3$ $\mathrm{J} / \mathrm{m} 2$ and $2.5 \mathrm{~J} / \mathrm{m} 2$ for $90 \mu \mathrm{m} 250 \mu \mathrm{m}$ and $390 \mu \mathrm{m}$ coating thicknesses, respectively. Figure 3.6. Image of fractured specimen. Bottom arm is the one with the epoxy layer below the thicker layer of residual glass. 37 Figure 3.7. Typical crack pattern observed under zero external load for coating thicknesses greater than $480 \mu \mathrm{m}$. The coating thickness in this image is $540 \mu \mathrm{m}$ 40 
Figure 4.1. Schematic depiction of the cracks emanating from a Vickers indent. $r$ is the half of the diameter length of the dent, and $a$ is the crack length measured from the center of the indent.

Figure 4.2. CTE for the SRT and BRT glasses, plotted along with the CTE of Ti6Al4V as reference $(n=3)$. Scatter bars indicate one standard deviation from the mean. The dashed lines are to help guide the eye.

Figure 4.3. Residual stresses experienced in the glass coating at the coating/substrate interface using the SRT and BRT glasses as coating $(n=3)$. Scatter bars indicate one standard deviation from the mean. The dashed lines are to help guide the eye.

Figure 4.4. Vickers hardness for the SRT and BRT glasses $(n=3)$. Scatter bars indicate one standard deviation from the mean. Stars and bars show statistical significance $(p<0.05) \ldots \ldots \ldots . .53$ Figure 4.5. Bulk Mode I critical strain energy release rates for the SRT and BRT glasses $(n=3)$. The $G_{I C}$ values for Fused silica glass and Pyrex obtained from the literature ${ }^{163,164}$ are also shown for reference. Scatter bars indicate one standard deviation from the mean. Stars and bars show statistical significance $(p<0.05)$.

Figure 4.6. SEM of a Vickers indent on SRT0 with the emanating cracks. The average half diameter and crack length are $54.8 \mu \mathrm{m}$ and $187.9 \mu \mathrm{m}$, respectively.

Figure 4.7. Mode I critical strain energy release rates for the coating/substrate systems with SRT and BRT glasses (3 samples per glass). Scatter bars indicate one standard deviation from the mean. Stars and bars show statistical significance $(p<0.05)$. 56 Figure.5.1. (a) Ti6Al4V bar coated with glass, (b) mode I setup for creating the mode I pre-crack (adapted from ${ }^{128}$ ), and (c) mode II setup. Figure is not to scale, i.e. the glass and epoxy layers are shown much thicker than they actually are for clarity.

Figure 5.2. Three steps in the analysis of residual stresses during cooling phase of the coating process based on $\mathrm{Yu}$ et al. ${ }^{136}$. $T_{\text {Coat }}$ refers to the temperature at which the bonding occurs, and $T_{\text {room }}$ is room temperature ${ }^{136}$.

Figure 5.3. The coefficient of thermal expansion (CTE) of the three glass compositions, with the CTE of Ti6Al4V as reference. For each glass composition, 3 specimens were tested. The error bars represent the corresponding standard deviation. The CTE of the Ti6A14V substrate was taken as $9.5 \times 10^{-6}$ based on the values in the literature ${ }^{157}$. 
Figure 5.4. Distribution of the residual stresses in the substrate and coating for representative samples of : (a, b) Ly-B0 coating system; (c, d) Ly-B3 coating system; and (e, f) Ly-B5 coating system. The normalized thickness is the distance from the coating/ Ti6Al4V substrate interface, $z$ (Figure.5.1), divided by the total thickness. The residual stresses are calculated from the deformed substrate profiles as explained in Section 5.2.3.2 ......

Figure 5.5. Critical strain energy release rate under mode I and mode II loading for three glass compositions. The mode I data is from Li et al. ${ }^{132}$ and the error bars represent the standard deviation for mode I tests and, min and max for mode II test results. 74 Figure 5.6. Image of two fractured specimens. Bottom arm is the adherend with the epoxy layer under the thicker layer of residual glass. 75 Figure 5.7. Residual layer thickness for mode I and mode II specimens. A lower value indicates a crack that is closer to the Ti alloy/coating interface. The error bars represent the standard deviation for both mode I and mode II test results. 76 Figure 6.1. Enamelled glass coating open faced specimens before degradation for (a) mode I and (b) mode II tests. In order to create a stable crack propagation condition, $13.9 \mathrm{~mm}$ (according to ASTM 3433134) and $38.9 \mathrm{~mm}$ (according to 171) of the glass was scraped off from the end of substrates 81

Figure 6.2. Double layer DCB used for mode I (a) ${ }^{128}$ and Mode II (b) ${ }^{129}$ fracture tests. 85 Figure 6.3. The XRD patterns of (a) the glasses and (b) glass coatings on the Ti6Al4V substrates, where the coating processing temperatures were tagged ${ }^{132}$...... .88 Figure 6.4. Weight loss of the coatings after different degradation times. The error bars show the standard deviation. The substrates used for the weight loss analysis were the as-received (not roughened) substrates 89 Figure 6.5. The thickness reduction (\%) due to degradation for Ly-B3 and Ly-B5 coatings in terms of weight loss (\%). The data points related to Ly-B0 (2h, 6h and 24h) and Ly-B3 (24h) data points are not shown here as these coating delaminated due to degradation even before any measurements could be done. The substrates used for the thickness change analysis were the asreceived (not roughened) substrates. 91

Figure 6.6. Ly-B0 sample delaminated after $2 \mathrm{~h}$ degradation. . 91

Figure 6.7. Critical strain energy release rate for coating/substrate systems made of Ly-B0 (a), Ly-B3 (b), Ly-B5 (c) in mode I and mode II loading condition. The data for undegraded samples 
(0h) is from Section 3.3.6 and Section 5.3.4 and the error bars illustrate the standard deviation for mode I tests and, min and max for mode II test results. Since Ly-B0 (2h, $6 \mathrm{~h}$ and $24 \mathrm{~h}$ ) and Ly-B3 (24h) coating delaminated prior to fracture testing, it was assumed that their $\boldsymbol{G I C}=\mathbf{0}$ and $\boldsymbol{G I I C}=0$. The substrates used for the fracture tests were the as-received (not roughened)

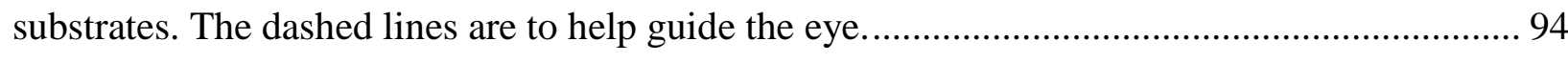
Figure 6.8. The thickness of the residual layer, $t_{r}$, for mode I (a) and mode II (b) samples. The error bars illustrate the min and the max for the $t_{r}$ results. The substrates used for the fracture tests were the as-received (not roughened) substrates. The lines are to help guide the eye. ........ 95 


\section{List of Abbreviations}

ASTM: American Society for Testing and Materials

CCIF: Cube Corner Indentation Fracture

CTE: Coefficient of Thermal Expansion

DCB: Double-Cantilever Beam

DSC: Differential scanning calorimetry

ENF: End Notched Flexure

FTIR: Fourier transform infrared spectroscopy

HA: Hydroxyapatite

ICDD: International Centre for Diffraction Data

IIF: Interface Indentation Fracture

PSA: Particle Size Analysis

VCOD: Vickers Crack Opening Displacement

VIF: Vickers Indentation Fracture

XRD: X-ray Diffraction Spectroscopy 


\section{Nomenclature}

\section{Roman symbols}

$\boldsymbol{a}$ : Distance from crack tip to the line of action of the loads

$a_{0}$ : Pre-crack length measured from pre-crack tip to the line of action of the loads

$\boldsymbol{A}_{c}$ : Cross-sectional area of the coating

$\boldsymbol{A l}_{\text {203: }}$ Aluminum Oxide

$\boldsymbol{A}_{s}$ : Cross-sectional area of the substrate

$\boldsymbol{b}$ : Half of the total thickness of the DCB specimen

$\mathbf{B}_{2} \mathbf{O}_{3}$ : Boron oxide

$\boldsymbol{C}_{\boldsymbol{1}}$ : An empirical function of the Dundurs parameters

CaO: Calcium oxide

CaCo3: Calcium carbonate

$\boldsymbol{d}$ : Distance from the line of action of the applied forces to the clamp at the root

$\boldsymbol{E}_{\boldsymbol{C}}:$ Young's modulus of the coating

$\boldsymbol{E}_{S}:$ Young's modulus of the substrate

$\boldsymbol{G}_{I C}:$ Mode I Critical Strain Energy Release Rate

$G_{\text {IIC }}$ : Mode II Critical Strain Energy Release Rate

$\boldsymbol{G}_{\boldsymbol{c}}$ : Apparent or global critical strain energy release rate

$\boldsymbol{G}_{\boldsymbol{R}}$ : Crack driving force (strain energy release rate) in the coating due to residual stresses

$\boldsymbol{H}$ : Hardness

$\boldsymbol{I}_{c}$ : Second moment of area of the coating's cross section

$I_{s}:$ Second moment of area of the substrate's cross section 
$I_{t}:$ Second moment of area of the cross section of the half-bilayer DCB specimen

$\boldsymbol{k}$ : Signed curvature of the coating/substrate system

$\boldsymbol{K}_{I C}:$ Mode I critical stress intensity factor

$\boldsymbol{K}_{c}$ : The contribution of the coating to the stiffness of the foundation of a half DCB specimen

$\boldsymbol{K}_{e}$ : The contribution of the epoxy to the stiffness of the foundation of a half DCB specimen

$\boldsymbol{K}_{s}$ : The contribution of the substrate to the stiffness of the foundation of a half DCB specimen

$\boldsymbol{L}:$ Half-chordal length of the coating /substrate system

$\boldsymbol{L}_{s}$ : Length of the substrate

$\boldsymbol{l}_{\boldsymbol{0}}:$ Initial length of the test specimen in CTE testing

$\boldsymbol{M}_{c}$ : Bending moment in the coating induced by residual stresses

$\boldsymbol{M}_{s}$ : Bending moment in the substrate induced by residual stresses

$\mathrm{Na}_{2} \mathrm{O}$ : Sodium oxide

$\mathrm{Na}_{2} \mathrm{CO}_{3}$ : Sodium carbonate

$P$ : load applied to the DCB specimen

$\boldsymbol{P}_{\boldsymbol{i n}}$ : The indentation load Vickers indentation test

$\boldsymbol{P}_{\boldsymbol{c}}$ : Axial force in the coating induced by residual stresses

$\boldsymbol{P}_{\boldsymbol{s}}$ : Axial force in the substrate induced by residual stresses

$\boldsymbol{P}_{\max }:$ Load required to propagate the crack

$\mathbf{P}_{2} \mathrm{O}_{5}$ : Phosphorus pentoxide

$r$ : Half of the diameter length of the dent in the Vickers indentation test

$\boldsymbol{R a}$ : Roughness Average

$\mathrm{SiO}_{2}$ : Silica

$\mathrm{SrCO}_{3}$ : Strontium carbonate 
$\boldsymbol{t}_{\boldsymbol{c}}$ : Thickness of the coating

$\boldsymbol{t}_{c r}$ : Critical coating thickness

$\boldsymbol{t}_{\boldsymbol{e}}$ : Thickness of the epoxy

$t_{r}$ : Thickness of the residual coating layer

$\boldsymbol{t}_{\boldsymbol{s}}$ : Thickness of the substrate

$T:$ T-stress

$\boldsymbol{T}_{\text {coat }}$ : Coating temperature

$\boldsymbol{T}_{g}$ : Glass Transition temperature

$\boldsymbol{T}_{i}$ : Room Temperature

TiN: Titanium Nitride

TiO2: Titanium dioxide

$T_{x}:$ Crystallization temperature

$w$ : Width of the coating and substrate

$W_{0}$ : The weight of Ti6Al4V bar before coating

$\boldsymbol{W}_{1}$ : The weight of enamelled Ti6Al4V bar

$\boldsymbol{W}_{2}$ : The weight of enamelled Ti6Al4V bar after degradation

$y$ : Offset of the crack plane with respects to the mid plane of the DCB specimen

$z$ : Distance of the layer of interest from the glass coating/ Ti6Al4V substrate interface

$Z$ : Crack driving force number

ZnO: Zinc oxide 


\section{Greek symbols}

$\alpha:$ First Dundurs parameter

$\alpha_{\text {calib}}$ : Calibration constant for the Vickers indentation test

$\alpha_{\text {glass }}$ : CTE of the glass

$\alpha_{m}$ : Mean CTE of the glass

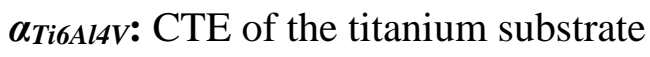

$\boldsymbol{\beta}$ : Second Dundurs parameter

$\delta$ : Adherends' deflection at the points of the applied load

$\boldsymbol{\delta}_{\boldsymbol{R}}$ : Maximum deflection of the substrate due to residual stresses

$\Delta l$ : Change in length of the sample

$\Delta T$ : Processing temperature

$\Delta W$ : The percentage of weight loss of enamelled Ti6Al4V bar due to degradation

$\boldsymbol{\kappa}_{\boldsymbol{c}}$ : Muskhelishwili's constant for the coating

$\boldsymbol{\kappa}_{s}$ : Muskhelishwili's constant for the substrate

$\boldsymbol{\mu}_{c}$ : Shear modulus of the coating

$\boldsymbol{\mu}_{s}$ : Shear modulus of the substrate

$v_{c}$ : Poisson's ratio of the coating

$v_{s}:$ Poisson's ratio of the substrate

$\left(\sigma_{c}\right)_{i n}$ : Maximum residual stresses in the coating at the coating/substrate interface

$\sigma_{M}$ : component of residual stress caused by the bending moment

$\sigma_{P}$ : component of residual stress caused by the axial force

$\sigma_{R}:$ Residual stresses

$\psi:$ Phase angle or mode ratio

$\psi_{L}:$ Phase angle of the loading

$\psi_{\boldsymbol{R}}$ : Phase angle of the crack driving force caused by the residual stresses 


\section{Introduction}




\subsection{Background}

Ti6Al4V is a common choice for the fabrication of orthopaedic implants () because of its high strength, biocompatibility, lack of toxicity ${ }^{1,2}$. However, there are several drawbacks associated with it. For example, the elastic modulus of Ti6Al4V is significantly higher than that of cortical bone which leads to stress shielding that might result in bone resorption and possible prostheses failure ${ }^{3}$. According to the literature, vanadium in Ti6Al4V may react with human tissue ${ }^{4}$. Finally, titanium alloys have been reported to fail to directly bond with bone ${ }^{5,6}$.

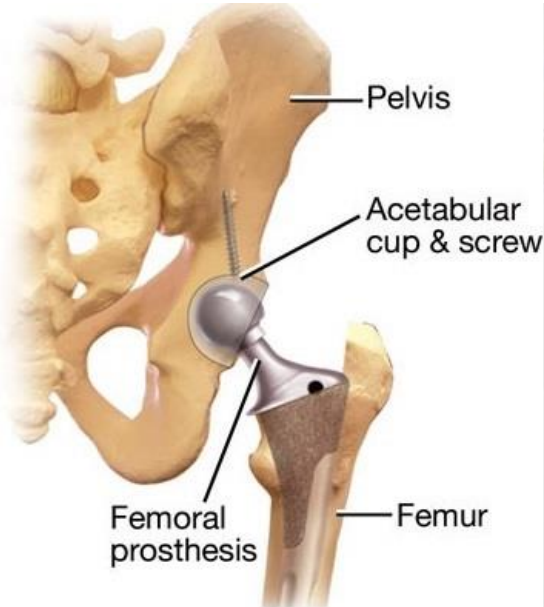

(a)

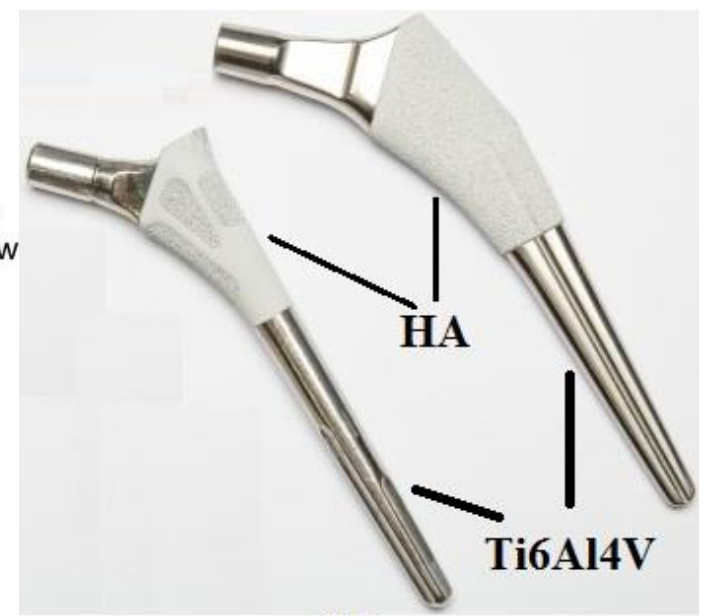

(b)

Figure 1.1. Use of Ti6AI4V orthopedic implants in hip replacement surgery $\left(a^{7}{ }^{7}\right.$. Hydroxyapatite (HA) is currently used as coating for these implants ${ }^{8}$.

In order to overcome these limitations, bioactive substances such as hydroxyapatite (HA) are often used as coatings for Ti alloy implants to improve the osseointegration and promote the stability of the implant/bone interface ${ }^{2,5,9,10}$. The major advantage of HA is that it is chemically similar to the apatite of natural bone and is a source of calcium and phosphate ${ }^{11}$. Sintered hydroxyapatite can bond to living bone after little degradation of the HA layer ${ }^{12}$. HA coatings have been reported to enhance new bone formation on an implant surface resulting in earlier and stronger fixation ${ }^{13,14}$. However, the long-term interfacial stability between these coatings and the Ti alloy substrate is rather poor. Significant loss ${ }^{5}$ of the HA coatings and interfacial fractures between HA coatings and the Ti6Al4V substrates have been observed after implantation ${ }^{15,16}$. These failures are primarily due to the residual stress induced by the mismatch of the coefficients of thermal expansion (CTE) of HA and Ti6Al4V which can lead to micro-cracking and de-bonding 
of the coating from the substrate $13,17,18$. Coating the titanium alloy with a bioactive glass is an alternative method to using HA. The potential of bioactive glasses as coatings was first postulated with the development of Hench's 45S5 Bioglass ${ }^{\circledR}$ in the $1960 \mathrm{~s}^{19}$. Bioglass ${ }^{\circledR}$ was the

first synthetic material to chemically adhere to both hard and soft tissue ${ }^{19}$. The use of bioactive glass coatings on medical implants is promising because of their propensity to release beneficial ions to the surrounding tissue, promoting osseointegration, antibacterial behavior, bone formation and growth, and tissue healing ${ }^{20-24}$. Bioactive glass coatings are also able to decrease implant corrosion and protect tissues against corrosion products ${ }^{25}$. Despite the above advantages, the widescale adoption of bioactive glass coatings has been limited because of both their relatively low toughness and their ability to adhere to metallic substrates ${ }^{25}$. Processing such glasses for use as coatings (e.g., through enameling ${ }^{26}$, plasma spraying ${ }^{27}$, electrophoretic deposition ${ }^{28,29}$, or glazing ${ }^{30}$ ) requires heat treatment to allow for the glass to react with the substrate surface thus creating a chemical bond ${ }^{31,32}$. Once the bond has formed and the assembly is cooled, a difference in CTE between the glass and metal will induce residual stresses, hence causing cracks to appear in the glass or at the glass/substrate interface.

\subsection{Literature Review}

In this section, a brief literature review is presented to facilitate a concise overview of the techniques to coat glasses onto metallic substrate, methods to measure the coating adhesion, and factors that affect the adhesion.

\subsubsection{Coating metallic implants with glass}

In terms of metal coating techniques, plasma spraying ${ }^{27}$ and enamelling ${ }^{10}$ are used more widely than other methods such as electrophoretic deposition ${ }^{28}$, and dip coating ${ }^{33}$. Schrooten and Helson ${ }^{27}$ applied a reactive plasma spraying method to coat Ti6Al4V plates with a 50 $\mu$ mlayer of silica-based bioactive glass. They reported the bonding strength to be sufficient for load bearing applications and concluded that the bonding strength between the glass and substrate depends on the strength of the glass itself. Even though coating by plasma spraying has been successfully used by some ${ }^{27,34-36}$, others have found this method may require high sintering temperatures that can lead to crystallization ${ }^{37,38}$, hence compromising the bioactivity of the 
coating. It has been also reported that plasma spraying can cause cracking in the glass or create poor adhesion in the glass/metal interface due to the high cooling rates involved ${ }^{39,40}$.

As an alternative, the enameling technique has been recommended due to its simplicity, and its capability to create a stronger bond between the coating and substrate ${ }^{10}$. For example, Chen et al. ${ }^{41}$ coated stainless steel samples with glass by spraying an ethanol based suspension of the glass on to the steel samples at room temperature. After drying the sample, they heat treated the assembly to help the glass spread along the substrate surface and bond with the substrate. They selected the heat treatment temperature well above the softening point to allow the glass to flow spread evenly on the substrate. Pavon et al. ${ }^{42}$, also used ethanol based glass suspension for coating silica glass onto metallic substrate, but instead of spraying the suspension, they deposited it onto the substrate. They air dried the samples and then heat treated them. Lotfibakhshaiesh et al. ${ }^{43}$ followed the coating procedure used by Pavon et al. ${ }^{42}$ with the only difference being a heat treatment of the samples in vacuum rather than air. They reported the coating procedure to be successful, confirming that depositing an ethanol based glass suspension onto the substrate is a promising coating method ${ }^{43}$. Mehdipour et al. ${ }^{28}$ applied the ethanol-glass suspension by means of electrophoretic deposition at constant voltages and then heat treated the samples to help with the sintering and bonding the glass with the substrate. They suggested avoiding high voltages in the deposition process since it increases the porosity and surface cracks. The effect of the heating profile in the enameling process was evaluated by Sola et al. ${ }^{26}$ who found that heating samples in a temperature ramp, up to the desired temperature, maintaining the temperature for $1 \mathrm{hr}$, and then letting the samples to slowly cool down can be very effective.

\subsubsection{Adhesion between bioactive glass coating and Ti6Al4V}

The degree of adhesion between a bioactive glass and a Ti6Al4V substrate depends on the glass composition and its structural characteristics which determine the nature of the van der Waals, electrostatic and /or chemical bonding forces between the coating and the substrate ${ }^{44}$. It also depends on the substrate surface texture and the procedure used to apply the coating. According to conventional enamelling theory, adhesion would be optimum if the glass layer neighbouring the metal were saturated with the lowest valence oxide of the metal without forming 
an interfacial layer ${ }^{45-47}$. Based on this theory, it is only then that a transition region will form between the metallic bonding of the alloy and covalent-ionic bonding of the glass, which provides a "continuity of electronic structure" 46 . Others have claimed that formation of nanostructured interfacial layers leads to optimum adhesion ${ }^{25}$.

\subsubsection{Measurement of coating adhesion}

There are a number of methods to characterize coating adhesion, both qualitative and quantitative. Qualitative methods such as pencil hardness tests and adhesion scratch tests can be performed rapidly and are well suited for comparative purposes ${ }^{48}$. Quantitative methods are mostly based on fracture mechanics and quantify the resistance of coatings or interfaces to crack growth, i.e., the fracture toughness or critical strain energy release rate required for crack propagation.

\subsubsection{Qualitative tests}

The majority of the literature on glass coatings has focused on qualitative or semiquantitative techniques in order to avoid the theoretical and experimental challenges facing the implementation of the quantitative counterparts. In the following subsections, the adhesion scratch test and indentation test will be briefly discussed.

\section{Adhesion scratch test}

The adhesion scratch test has been commonly used ${ }^{44,49,50}$ to characterize the adhesion between a coating and substrate in terms of the critical normal load under which a moving diamond

stylus creates a well-defined failure on the surface of the coating ${ }^{51}$ (Figure 1.2). This quantity by itself is useful only for comparing the bonding strength of different coatings with the same thickness ${ }^{48}$. 


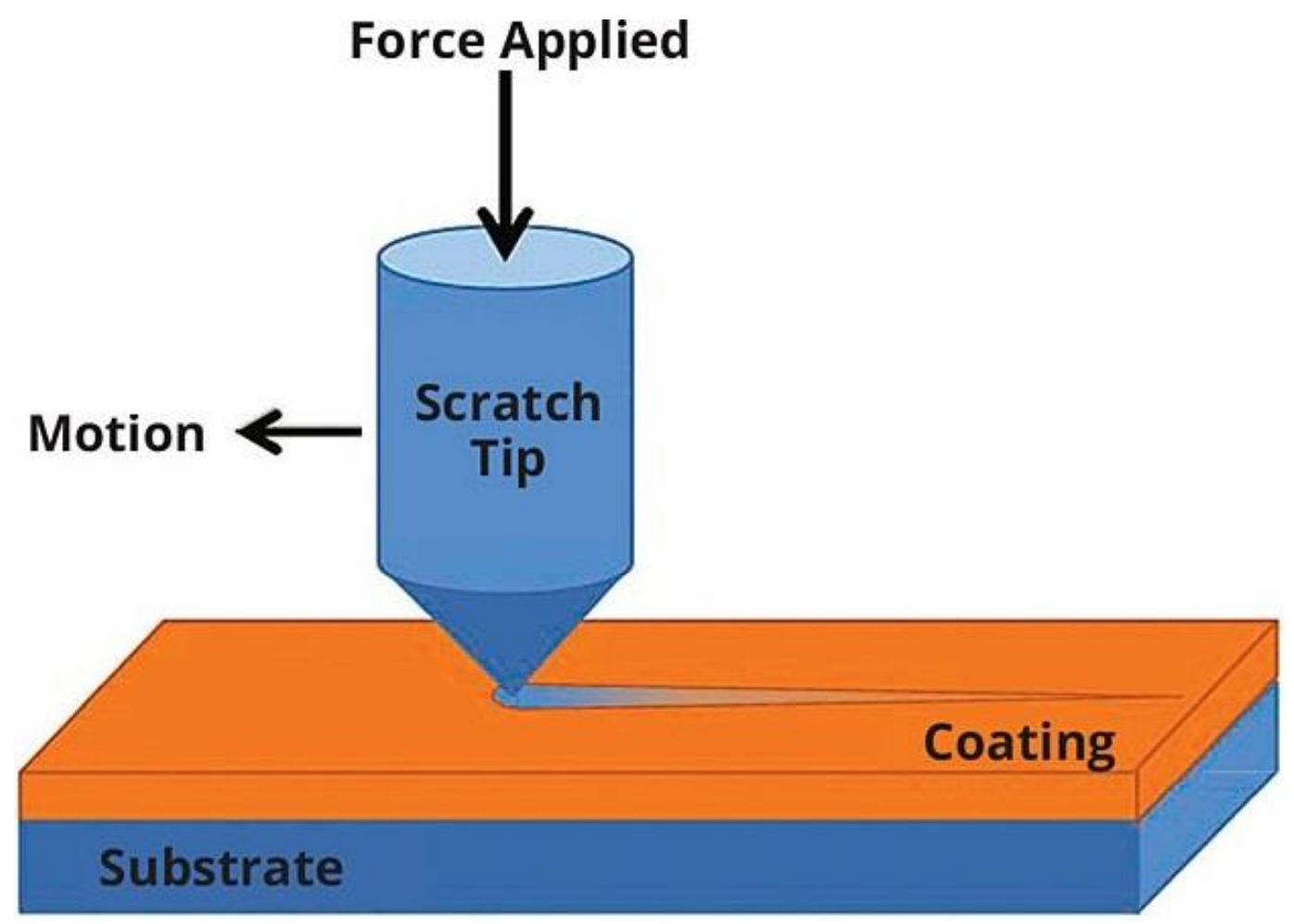

Figure 1.2. Adhesion scratch test ${ }^{52}$ quantifies the adhesion between the coating and substrate by measuring the critical normal force under which a moving diamond stylus creates a well-defined failure on the surface of the coating.

This method was first introduced by Heavens ${ }^{53}$ in 1950 and has been often used since, as a simple, practical and rapid method ${ }^{44,50,54}$. However, this technique does not measure the fundamental adhesion strength of the bonding. It only gives an engineering measurement of the practical and intrinsic adhesion between the coating and substrate ${ }^{51}$. According to ASTM C1624 "Standard Test Method for Adhesion Strength and Mechanical Failure Modes of Ceramic Coatings by Quantitative Single Point Scratch Testing" "51, this test method is not recommended for coatings thicker than $30 \mu \mathrm{m}$. Furthermore, some researchers have criticized its use for systems in which the coating-substrate adhesion is not the only factor that contributes to the measured critical load ${ }^{55,56}$. For example, Von Stebut et al. ${ }^{55}$ showed that for hard coatings on hard substrates, the scratch test damage is also in part due to the cracks initiated along the track edges and therefore, suggested that analyzing such cases requires fracture mechanics modelling. There are also concerns with scratch tests for thin hard coatings on soft substrates, since the coating is prone to fracture due to stresses caused by the substrate deformation ${ }^{50}$. 


\section{Indentation test}

Lardner et al. examined the crack propagation path near a free surface and near the interface of a glass to epoxy bond by inducing a crack using the indentation test ${ }^{57}$. Gomez-Vega et al. ${ }^{1}$ and Lopez-Esteban et al. ${ }^{25}$ both studied the crack path resulting from indentation in order to compare the resistance of the interface with that of the glass. However, the results in both cases were described in qualitative terms, i.e. the former characterized the bond as "good adhesion" and the latter as a "strong bond". In other words, the adhesion was characterized as strong if the crack tended to grow in the glass, rather than the glass/metal interface (Figure 1.3). Such evaluations, also used by other researchers, ${ }^{5,58-62}$ are somewhat useful when the effects of a change in a single parameter (e.g. thickness, substrate pre-treatment, etc.) of a coating system are compared with all other parameters constant, or when rankings of coating performance of similar systems are required. However, they cannot reliably be used to compare the adhesion for different systems because they do not fundamentally and quantitatively measure adhesion. Such challenges have led investigators such as Lopez-Esteban et al. ${ }^{5}$ to stress the need for more exhaustive characterization.

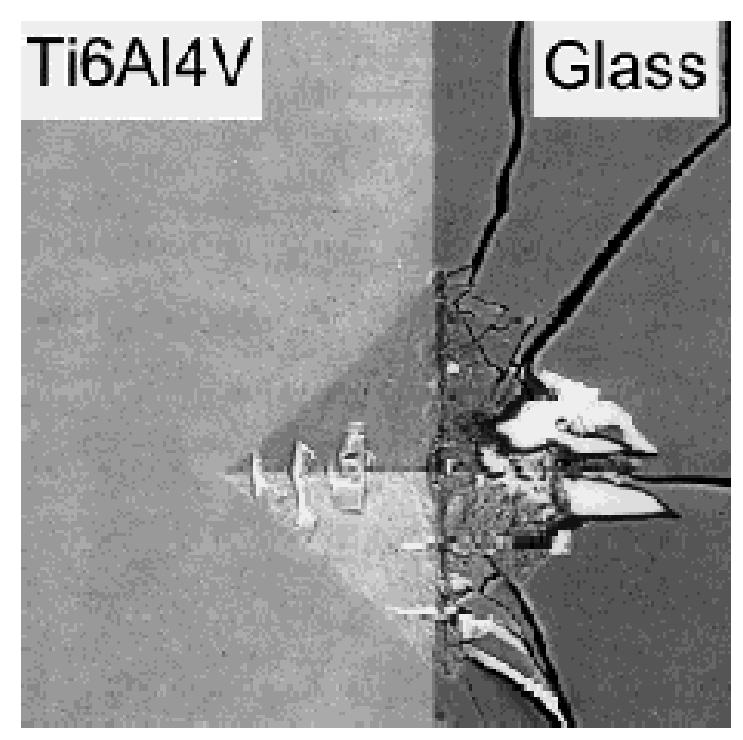

Figure 1.3. Qualitative indentation test ${ }^{10}$ characterizes the adhesion as strong if the crack emanated from an interfacial indent grows in the coating rather than the coating/substrate interface. 


\subsubsection{Quantitative tests and fracture mechanics approach}

According to the energy approach used in linear elastic fracture mechanics, crack extension occurs when the energy available for crack growth is sufficient to overcome the resistance of the material to crack propagation. The material resistance includes, but is not limited to, the surface energy and plastic work. The energy criterion was proposed first by Griffith ${ }^{63}$, however Irwin 49 is the one who developed the present version of this approach. The energy release rate, $G$, is defined as the rate of change of potential energy with respect to the crack area for a linear elastic material. Fracture occurs when $G=G_{c}$, where $G_{c}$ is the critical energy release rate, a measure of the fracture toughness.

There are three different modes of fracture. In mode I, also known as the "opening mode", the displacements of the crack surfaces are perpendicular to the plane of the crack. In mode II or the "sliding mode", the displacement of the crack surfaces is in the plane of the crack and perpendicular to its leading edge. Finally, the mode III or the "tearing mode" occurs when there is an out-of-plane shear. In that case the crack surface displacements are in the plane of the crack and parallel to the crack leading edge ${ }^{64}$.

Cracks in bonded systems can be divided into two different groups. If the cracks grow entirely within the adhesive layer then they are considered as cohesive. This is generally desirable, since it means that the bonding between the adhesive and adherend is tougher than the adhesive itself. However, if cracks run along the interface between two materials, this causes adhesive fracture. In the case of adhesive failure, contrary to cohesive failure, the cracks are not generally free to evolve as mode I cracks, and mixed-mode fracture concepts (combinations of tension and shear) have to be considered. If the presence of the adhesive layer itself is ignored and the adherends differ, then any resulting failure would be adhesive on the macroscopic scale ${ }^{48}$.

As discussed earlier, quantitative methods are mostly based on fracture mechanics and quantify the resistance of coatings or interfaces to crack growth. In the following subsections, indentation based fracture testing, tension testing, pull out test and beam specimens will be briefly discussed. 


\section{Indentation based fracture testing for bulk coatings}

There have been efforts to quantify fracture toughness from direct measurements of cracking in an indentation test. For example, the Vickers indentation fracture (VIF) test ${ }^{58,61}$, the cube corner indentation fracture (CCIF) test ${ }^{59}$, the Vickers crack opening displacement (VCOD) test ${ }^{60,65,66}$ and the interface indentation fracture (IIF) test ${ }^{62}$ have all been considered. However, Kruzic et al., ${ }^{67}$ and Hsiung et al., ${ }^{68}$ have advised to be cautious when using such methods to characterize fracture toughness $\left(K_{I c}\right)$ because, especially for brittle coatings, they are prone to large errors due to violations of model assumptions during practical testing scenarios.

Among all of the indentation techniques used for assessing the fracture toughness, Vickers indentation fracture (VIF) test (Figure 1.4) is by far the most common technique which is based on the direct measurement of the lengths of cracks emanating from Vickers indents. This technique was first developed by Lawn et al., ${ }^{61}$ under the assumption that such cracks are created due to tensile stresses that form during unloading. Anstis et al., ${ }^{58}$ validated Lawn's model for several ceramics and glasses by comparing the fracture toughness obtained from the VIF test with that from standard fracture tests. Later, Laugier ${ }^{69}$ showed that indentation crack geometry in glasses and ceramics are different and claimed that Lawn's model requires some modification when used for evaluation of ceramic toughness, and therefore developed a new model that describes the indentation cracking in ceramics more realistically. Kruzic et al., ${ }^{67}$ claimed that the VIF technique in some cases might result in toughness values with $\sim 50 \%$ error and recommended using this technique for comparative purposes only, while considering that in order to observe a significatnt difference between the toughness values, they must differ at least by a factor of three. 


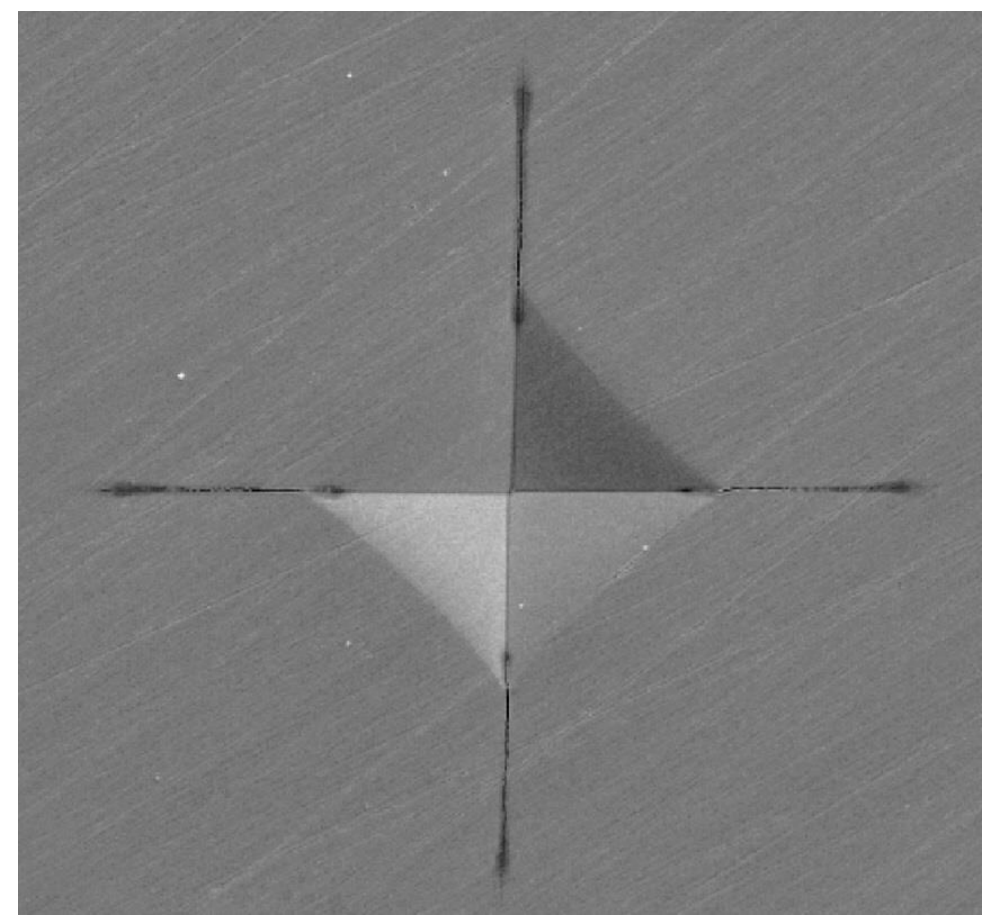

Figure 1.4. Vickers indentation fracture test ${ }^{70}$ measures the fracture toughness from direct measurements of cracking in an indentation test.

\section{Tension testing technique}

Braem et al. ${ }^{71}$ measured the tensile adhesion bonding strength by preparing a test assembly which consisted of an uncoated and coated Ti alloy disc of the same dimensions bonded together using a very strong adhesive (ASTM F1147 ${ }^{72}$ ) (Figure 1.5). They used a tensile machine to pull the specimen apart and characterized the adhesion strength as the nominal failure tensile stress in the coating, i.e. the maximum load per unit cross sectional area. Berndt and Lin ${ }^{73}$ used the same technique and concluded that such testing methods do not provide a fundamental understanding of coating performance, and recommended taking a fracture mechanics approach instead. In another approach, Baino and Vitale-Brovarone ${ }^{74}$ and Chen et al. ${ }^{75}$ developed a mechanical model that uses the experimental data obtained from the tensile test to predict the fracture toughness of the coatings. 


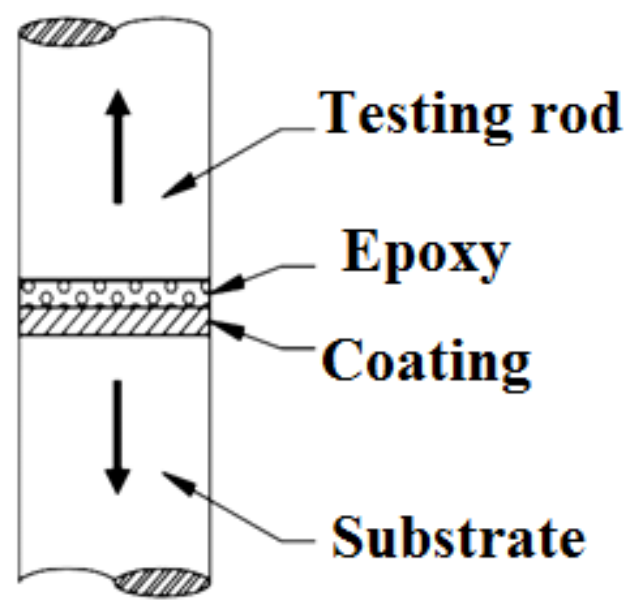

Figure 1.5. Tension testing technique.

\section{Pull out test for measuring the shear adhesive strength}

The coatings on biomedical implants such as hip and knee prostheses are usually subjected to a combination of both shearing (mode II) and opening (mode I) loads ${ }^{36}$. The relative magnitude of mode I and II components depends on several factors including loading conditions, the shape and elastic properties of the implant, the structure of the surrounding bone and the roughness at the interface. In the case of knee implants, the mode II component is critical because the tibial component of the implant experiences high torques and shear forces which are reported to cause coating delamination and consequently loosening ${ }^{36}$. Therefore, improving the shear strength of such coating/substrate systems could result in better long-term fixation between the prosthesis and the bone ${ }^{76}$. A commonly used method to assess the performance of coated implants under shear loading is the push-out test (e.g., ${ }^{77}$ ) (Figure 1.6) in which a coated cylinder representing the implant is mechanically pushed out of a bone sample until failure occurs. The load at failure divided by the interfacial contact area provides an approximate measure of interfacial shear strength. This technique has been used mostly to evaluate the bone/coated implant adhesion ${ }^{11,77-}$ ${ }^{80}$ and only provides a semi-quantitative measure of adhesion since the stresses are not uniform within the coating or interface. 


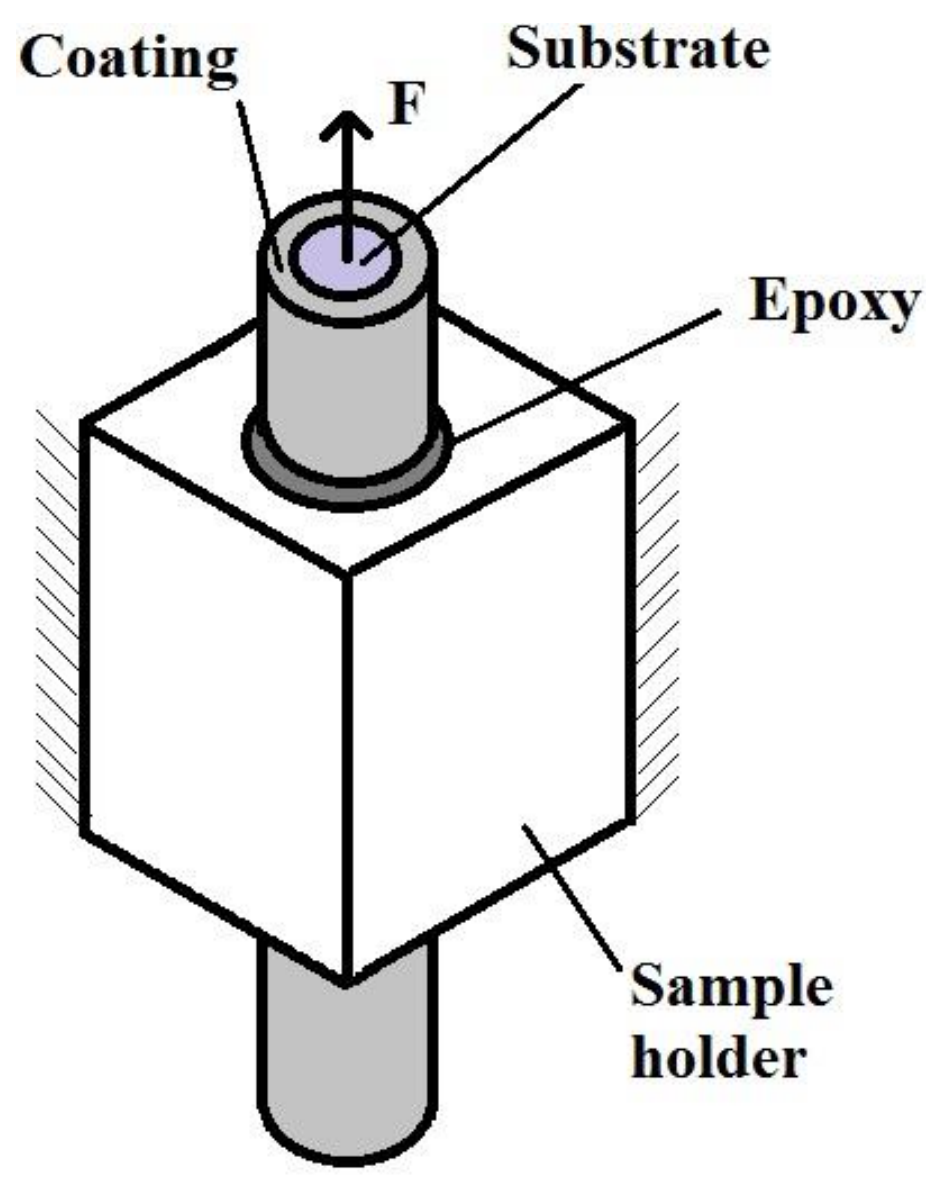

Figure 1.6. Pull out test ${ }^{81}$.

\section{Pure mode I and mode II fracture testing using beam specimens}

There are several fracture testing methods to quantitatively measure the adhesion strength between the bioactive glass and metallic substrate. In such techniques adhesion is quantified in terms of a measured critical strain energy release rate under mode I $\left(G_{I C}\right)$, and mode II $\left(G_{I I C}\right)$ loading conditions. The double cantilever beam (DCB) (Figure 1.7) is the most common specimen for measuring the critical strain energy release rate ${ }^{82}$. In DCB specimen, the layer to be tested is sandwiched between two identical adherends. Usually a pre-crack is initially introduced to the DCB specimen. The advantage of this specimen is that it allows the mode I, mode II or mixed mode fracture toughness to be measured using different loading configurations. If the two beams are pulled apart at one end with the same load on each arm, the critical strain energy release rate in mode I condition can be measured (Figure 1.7 (a)). If the two beams are clamped at one end and loaded at the other with equal loads applied in the same direction, the mode II critical strain energy 
release rate can be calculated (Figure 1.7 (b)). Finally, if the two beams are pulled apart with unequal forces while the other end is clamped, a combination of mode I and mode II fracture resistance can be measured (Figure 1.7 (c) ${ }^{82}$. Beam type fracture specimens will be discussed in more detail in Sections 3.2.5.3 and 5.2.3 .

(a)

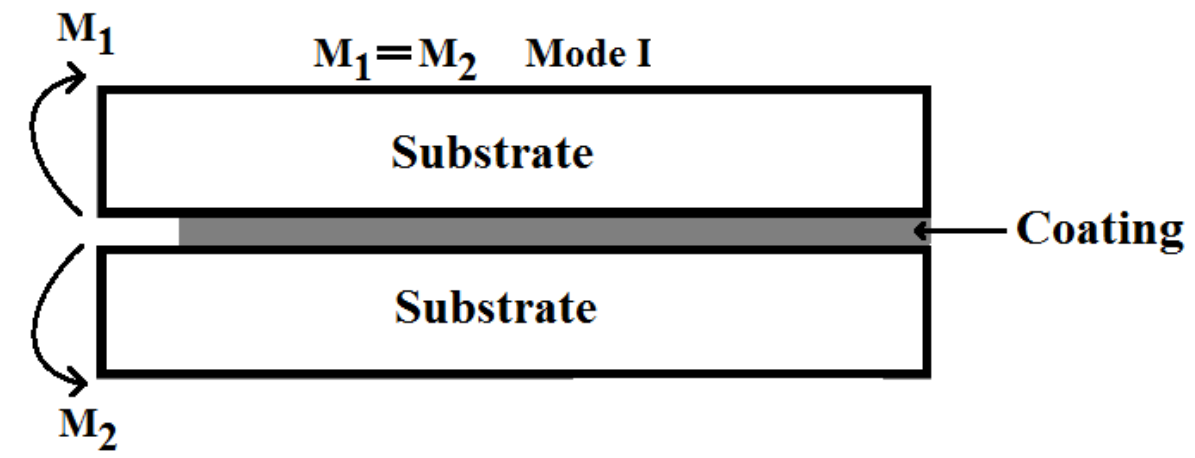

(b)

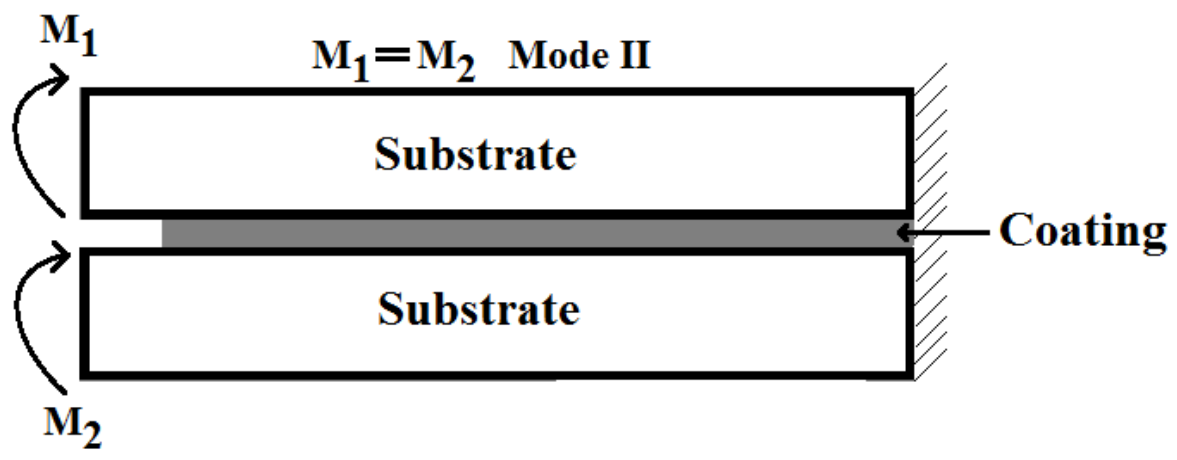

(c)

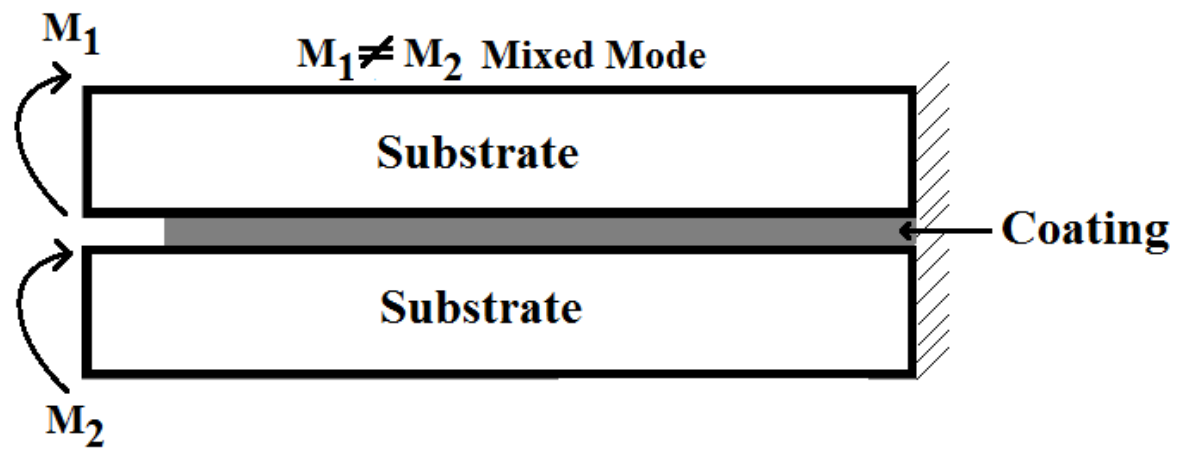

Figure 1.7. Double Cantilever Beam (DCB) Specimen for mode I (a), mode II (b) and mixed mode (c) fracture testing.

In fracture testing of relatively tough layered materials, it is often found that there is an " $\mathrm{R}$ curve" behaviour in which the $G_{I C}$ values from the initial pre-crack are initially very low, but gradually increase to a steady state value after a number of crack propagations, as a toughened "damage zone" forms and grows to a steady state size ${ }^{64,83,84}$. For brittle materials such as glasses 
however, such R-curve behaviour does not exist ${ }^{82}$ since the damage zone is fully developed within the first crack propagation. In Chapter 3 and Chapter 5 of the present dissertation, a modified version of DCB specimen was developed that allowed quantifying the $G_{I C}$ and $G_{I I C}$ of bioactive glasses enamelled on metallic substrates.

\subsubsection{Effect of coating thickness on adhesion}

There have been efforts to evaluate the effect of coating thickness on the adhesion. Jindal et al. ${ }^{85}$ conducted series of experiments on physically vapored TiN coatings of different thickness (4-16 $\mu \mathrm{m}$ ) by ion plating on a cemented carbide substrates. After performing the scratch test to evaluate the adhesion, they concluded that there was no clear correlation between the adhesion and coating thickness. Takadoum and Bennani ${ }^{86}$ also worked on TiN films, but they were deposited on steel substrates. They used the scratch test on coatings of 1.5, 3 and $5 \mu \mathrm{m}$ thickness and reported an inverse relationship between coating thickness and adhesion.

Malzbender and de With ${ }^{87}$ investigated the effect of coating thickness in hybrid organicinorganic coatings deposited by spin coating onto float glasses. They created coatings of various thickness $(1-20 \mu \mathrm{m})$ and observed that thicker coatings had larger residual stresses and therefore they were more prone to cracking. This is in agreement with Schmidt ${ }^{88}$, who worked on similar coating/ substrate system and concluded that the coating thickness will increase the probability of cracking. This result is different from what has been discovered for rubber-modified epoxy adhesives, where the critical strain energy release rate initially increases with bondline thickness, then decreases and eventually reaches a constant value ${ }^{89-93}$. Since no previous study has investigated the effect of coating thickness on the mechanical performance of bioactive glass coatings, Chapter 3 of the present dissertation has focused on measuring the fracture toughness of bioactive glasses with different thicknesses coated on Ti6Al4V substrates.

\subsubsection{Effect of glass composition on adhesion}

The presence of potentially large residual stresses introduced during the high temperature glass coating process further affects coating performance and the adhesion between the coating and the substrate. These large residual stresses can be caused during the coating process due to the 
mismatch of the coefficients of thermal expansion (CTE) of the coating and substrate. These residual stresses can be sufficient to cause delamination or cracking of the coating ${ }^{5,94}$. Bioactive glasses are most commonly made with a silica content of less than 60 wt. \% to facilitate osseointegration and increase reactivity in vitro and in vivo ${ }^{95}$. Since the CTEs of bioactive glasses are typically higher than that of Ti alloys ${ }^{25}$, a number of researchers have proposed to tailor the composition of the glass to reduce this difference. For example, it has been suggested that increasing $\mathrm{SiO}_{2}$ content may reduce CTE to acceptable levels, but this also leads to an undesirable reduction in glass bioactivity ${ }^{20,96,97}$. Gomez-Vega et al. suggested solving this problem by creating multilayer coatings, one layer made from a high Si content glass to lower the CTE, and the second layer made from a low Si content glass to compensate for reduced bioactivity ${ }^{10}$. However, they observed that since the substrate thickness was usually large compared to that of the glass, whenever the second layer of the coating had a $\mathrm{SiO}_{2}$ content lower than $53 \mathrm{wt} \%$, it tended to crack. A change in the glass backbone may reduce CTE, but this change can deleteriously affect other characteristics of the glass, such as increasing glass transition $\left(T_{g}\right)$ and crystallization $\left(T_{x}\right)$ temperatures ${ }^{40}$. Recently, borate-based bioactive glasses have been considered for biomedical applications ${ }^{98-101}$. These glasses are promising candidates for coating titanium alloy substrates because they can be tailored to bond more strongly to the metal compared to their silicate counterparts ${ }^{5,102}$. They can also be designed to have similar coefficients of thermal expansion (CTE) to the titanium alloy ${ }^{103}$, thus minimizing residual stresses that can lead to the coating cracking ${ }^{17}$. These challenges make it clear that methodologies are necessary to quantify the coating adhesion for different glass compositions in the presence of residual stresses. To address this need, Chapter 4 of the present study has investigated the effect of composition on the mechanical performance of the bioactive coatings by measuring the fracture toughness of boratebased and silica-based coatings with different amounts of incorporated $\mathrm{TiO}_{2}$.

\subsubsection{Effect of degradation of the bioactive glass on adhesion}

In-situ degradation of bioactive glasses makes them desirable for clinical applications because of their ability to release beneficial ions to the surrounding tissues promoting antibacterial behavior, bone formation and growth, tissue healing, etc. ${ }^{21-24}$. This degradation can affect the 
mechanical performance of bioactive glass coatings considerably, and it is therefore important to measure the adhesion and fracture toughness of the bioactive glass coatings after degradation.

In an effort to study the effect of degradation on the mechanical performance of glass coating/substrates, Pavon et al. ${ }^{104}$ used an indentation based method, focusing on the fatigue behaviour of silica based bioactive glass coating/ Ti6Al4V, and reported that degradation of the samples in water results in cracking at lower cyclic loads. They attributed this effect to the presence of water molecules which break the silica-oxygen bond ahead of the crack tip indentation. In another study, Huang et al. ${ }^{105}$ left samples made of 58S bioactive glass coating/AZ31 substrates in Simulated Body Fluid (SBF) for 15 hours, and after observing no obvious peeling off, concluded that the adhesion was strong after degradation.

Investigators studying the related area of HA coatings have usually used qualitative or semi-quantitative approaches to study adhesion rather than applying quantitative fracture mechanics methods. For example, it has been reported that the bond strength between HA coatings and Ti6Al4V substrates significantly declined due to the degradation of HA coatings after they were immersed in SBF ${ }^{106}$ or implanted in vivo ${ }^{107}$. However, in these studies ${ }^{106,107}$, adhesion was measured in terms of the failure tensile stress of the coating bonded to a loading rod, which did not provide a fundamental measure of toughness.

Although the materials and adhesion mechanisms are different than in bioactive glasses, useful information regarding testing methodologies for degraded multilayer systems can be found in the literature on epoxy structural adhesive joints. For example, Wylde and Spelt ${ }^{108}$ used openfaced epoxy adhesive/ aluminum alloy specimens subject to moisture and heat in order to greatly accelerate the rate at which the adhesive degraded. Ameli et al. ${ }^{109,110}$ used a similar approach to study the fracture properties of a degraded rubber-toughened epoxy adhesive/aluminum alloy system, also determining its fracture R-curve. In another work by Ameli et al. ${ }^{111}$, they showed that data from such accelerated aging tests can be used to predict the durability of the adhesives under service conditions. They also showed that the different water absorption/desorption behaviours of adhesives can be related to the degradation of the fracture properties ${ }^{112}$. Datla et al. ${ }^{113}$, studied the mixed-mode fatigue behavior of toughened epoxy-aluminum adhesives after being exposed to 
either a constant humidity environment or a cyclically changing environment. They illustrated that the samples under cyclic humidity show superior fatigue performance.

The degradation rate of bioactive coatings is another important issue. Common silicate bioactive glasses such as 45S5 degrade very slow compared to the formation rate of new tissues 114. The slow degradation rate can cause in vivo stability concerns because the remaining glass remains unconverted to HA. Borate bioactive glasses convert to HA faster and more completely due to their low chemical durability ${ }^{115,116}$. Nevertheless, the high dissolution rate may induce cell damage ${ }^{117-119}$ and loss of the coating-substrate bond strength, subsequently retarding implant fixation ${ }^{36}$. In order to address the problem, in Chapters 5 and 6 of the present dissertation, Strontium ions $\left(\mathrm{Sr}^{2+}\right)$ were incorporated into the borate-based glasses to modify the dissolution rate $^{120}$, increase the proliferation of osteoblast cells in vivo ${ }^{121}$ and stimulate bone formation in vitro ${ }^{122}$. Also, since no previous study has investigated the mechanical performance of bioactive coatings, Chapter 6 of the present dissertation has focused on measuring the fracture toughness of bioactive glass coatings after degradation.

\subsubsection{Effect of substrate roughness on adhesion}

The substrate surface texture can significantly affect the adhesion between the coating and the substrate; for example, it is well known that changes in roughness can affect adhesion ${ }^{86,123-}$ 127. Takadoum and Bennani ${ }^{86}$ investigated the effect of roughness on adhesion by coating TiN films by reactive ion plating on stainless steel substrates with various surface roughness ranging from $R_{a}=0.02 \mu \mathrm{m}-0.35 \mu \mathrm{m}$. They used the scratch test on coatings of $1.5,3$ and $5 \mu \mathrm{m}$ thickness and reported that adhesion on the smoother substrates was better than the rougher ones. In order to explain this phenomenon, they studied the coating/substrate interface during the scratch test and suggested that roughness peaks are easier to break when they are higher and therefore removing the coating will be easier for rougher substrates. This conclusion is in agreement with the study of Steinmann et al. ${ }^{123}$. In another study DeBryun et al. ${ }^{124}$ observed that adhesion of high coating thickness/high substrate roughness systems is comparable to that of low coating thickness/low substrate roughness which suggests that coating thickness/substrate roughness ratio is the determining factor rather than the roughness itself. 
Fukanuma and Ohno ${ }^{125}$ created different ranges of roughness $\left(R_{a}=1.4 \mu \mathrm{m}-3 \mu \mathrm{m}\right)$ on aluminum and steel plates and then plasma sprayed white alumina on these substrates to create a $500 \mu \mathrm{m}$ layer coating. They concluded that increasing the roughness improved the adhesion between the coating and substrate. Wang et al. ${ }^{126}$ reached the same conclusion after studying the effect of roughness ranging from $\mathrm{R}_{\mathrm{a}}=0.059 \mu \mathrm{m}-9 \mu \mathrm{m}$ in $\mathrm{NiCrBSi}$ and WC-Co high velocity oxy fuel sprayed coatings. They explained this phenomenon by pointing out the fact that the bonding mechanism in this coating/substrate system was predominantly mechanical interlocking. Since no previous study has investigated the effect of substrate roughness on the mechanical performance of bioactive glass coatings, Chapter 6 of the present dissertation has focused on measuring the fracture toughness of bioactive glasses coated on Ti6Al4V plates with different substrate roughnesses.

\subsection{Motivation}

The literature on bioactive glass coatings has focused on glass formulation and characterization, evaluation of biocompatibility, osseointegration and degradation and chemical analysis of the interface. An often neglected, but equally important, factor is the coating's mechanical performance. For example, according to Lopez-Esteban et al. poor coating adhesion and toughness remain barriers to wide scale adoption of glass coatings ${ }^{25}$. Therefore, evaluation of coating adhesion and toughness needs to be considered an essential part of the development of new glass coatings.

Most of the applied techniques for characterizing the adhesion of bioactive glass coatings to metals in the literature give only qualitative or semi-quantitative information that can be useful for comparison purposes. The presence of potentially large residual stresses introduced during the high temperature glass coating process further complicates matters since it may significantly affect coating performance.

This work performs a rigorous fracture mechanics study on bioactive glass coatings for metallic substrates. In order to quantify the fracture toughness of glass/metal system, new fracture mechanics testing methodologies are postulated. Using these fracture testing techniques, the effect of glass coating thickness, glass composition, substrate roughness, loading condition (fracture 
mode) and glass degradation on the fracture toughness of the coating/substrate system is studied. In addition, the residual stresses in the glass caused by the mismatch in the coefficient of thermal expansion between the glass coating and the metallic substrate is measured and effect of the residual stresses on the fracture toughness, crack path selection and crack propagation stability is studied.

\subsection{Objectives}

This work aims to perform a rigorous fracture mechanics study on bioactive glass coatings for metallic substrates. The primary objectives of this research are the following:

1. Investigate the effect of glass coating thickness on the fracture of the coating/substrate system under mode I loading. This primary objective will be achieved by meeting the following secondary objectives:

- Develop a reliable and reproducible coating technique to create a uniform layer of bioactive glass coating onto the metallic substrate.

- Develop a novel methodology for quantifying the critical strain energy release rate of enamelled bioactive glass coatings under mode I loading condition. This testing technique will allow quantifying the fracture toughness of the glass/metal system in terms of a mode I measured critical strain energy release rate $\left(G_{I C}\right)$.

- Develop a method to measure the residual stresses in the glass caused by the mismatch in the coefficient of thermal expansion (CTE) of the glass coating and the metallic substrate. Once this objective is achieved, the effect of residual stresses on the fracture of the coating/substrate system can be investigated.

2. Investigate the effect of glass composition on the fracture of the coating/substrate system. This objective will be achieved by creating specimens made of borate-based and silicabased coatings with different amounts of incorporated $\mathrm{TiO}_{2}$ and $\mathrm{SrCO}_{3}$ and testing them by applying the developed fracture testing methodology.

3. Investigate the effect of loading condition on the fracture of the system. This primary objective will be achieved by meeting the following secondary objectives:

- Develop a methodology for quantifying the critical strain energy release rate of enamelled bioactive glass coatings under mode II loading condition which will allow 
quantifying the fracture toughness of the glass/metal system in terms of a mode II critical strain energy release rate $\left(G_{I I C}\right)$.

- Create specimens made of glass coatings with different compositions and testing them by applying the developed mode II testing methodology and compare the measured $G_{I I C}$ values with their $G_{I C}$ counterparts.

4. Investigate the effect of degradation on the fracture of the coating/substrate system. This primary objective will be achieved by meeting the following secondary objectives:

- Develop an experimental procedure to simulate the degradation of the bioactive glass coating in an environment that simulates human body.

- Use this developed experimental procedure to evaluate the effect of degradation on the fracture of the glass coatings.

5. Investigate whether roughness is a relevant parameter in studying the properties of the coating substrate system. This primary objective will be achieved by meeting following the secondary objectives:

- Develop a procedure to create three levels of substrate roughness using abrasive jet machining.

- Perform fracture testing on specimens made of glasses coated on roughened substrates to investigate whether the fracture toughness of the system can be improved by increasing roughness.

\subsection{Author's contribution in the context of collaboration}

The fracture testing specimens (DCB samples) for mode I and mode II tests used for Chapters 3 through 6 were all prepared by the author. The measurements and analysis of thickness (Chapters 3 through 6), roughness (Chapters 3, 5 and 6) and residual stresses (Chapters 3, 5 and 6) were also performed entirely by the author. The author also performed and analyzed all of the fracture tests (mode I (Chapters 3 through 6), mode II (Chapters 5 and 6) and Vickers indentation fracture (Chapter 4)) in this dissertation. 
The preparation of the coatings was performed partially by the author. CTE measurements of Chapter 4 and 5 were conducted by trained personnel and analyzed partially by the author as well.

Glass preparation and characterization (X-ray diffraction (Chapter 3 and 6), particle size analysis (Chapter 3), and differential scanning calorimetry (Chapter 3) tests) were performed entirely by colleagues. Preparation of the discs (Chapter 4 and 6), measuring the hardness (Chapter 4) and analysis of weight loss (Chapter 6) were carried out by colleagues as well. The calculations of residual stresses in Chapter 3 were also performed by a colleague. 


\section{Sample preparation}




\subsection{Introduction}

This chapter introduces the nomenclatures and compositions of the glasses used in this dissertation, as well as the process of manufacturing them. It also presents the procedure of coating the substrates with the manufactured glass.

\subsection{Glass preparation}

The Table 2.1 presents the nomenclatures and compositions of the glasses. In SRT and BRT glass series, $\mathrm{TiO}_{2}$ was added at the expense of $\mathrm{SiO}_{2}$ and $\mathrm{B}_{2} \mathrm{O}_{3}$, respectively. For Ly-B series, $\mathrm{SrCO}_{3}$ was incorporated in the composition at the expense of $\mathrm{B}_{2} \mathrm{O}_{3}$. One batch of each glass was manufactured by firing the appropriate amounts of analytical grade reagents (Sigma-Aldrich, Oakville, ON, Canada \& Fisher Scientific, Ottawa, ON, Canada) for $1 \mathrm{hr}$ at the appropriate temperature (1400-1500 ${ }^{\circ} \mathrm{C}, 1200^{\circ} \mathrm{C}$ and $1300^{\circ} \mathrm{C}$ for SRT, BRT and Ly-B, respectively) in silica crucibles and shock quenched in cool water. The resulting frit was then ground and sieved to retrieve glass powders with a mean particle size of $\leq 20 \mu \mathrm{m}$.

Table 2.1. Glass formulations.

\begin{tabular}{|c|c|c|c|c|c|c|c|c|c|}
\hline \multirow[b]{2}{*}{ 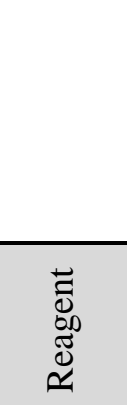 } & \multicolumn{3}{|c|}{$\begin{array}{c}\text { SRT } \\
\text { Silica-based } \\
\text { glasses with } \\
\text { varying } \mathrm{TiO}_{2} \\
\text { (mol\% weight) }\end{array}$} & \multicolumn{3}{|c|}{$\begin{array}{c}\text { BRT } \\
\text { Borate-based } \\
\text { glasses with } \\
\text { varying } \mathrm{TiO}_{2} \\
\text { (mol\% weight) }\end{array}$} & \multicolumn{3}{|c|}{$\begin{array}{c}\text { Ly-B } \\
\text { Borate-based glasses } \\
\text { with varying } \mathrm{SrCO}_{3} \\
(\mathrm{~mol} \% \text { weight })\end{array}$} \\
\hline & $\begin{array}{l}0 \\
\frac{1}{\sigma} \\
\frac{1}{n}\end{array}$ & $\bar{E}$ & $\begin{array}{l}n \\
\frac{n}{\sigma}\end{array}$ & $\begin{array}{l}\circ \\
\stackrel{\underline{v}}{n} \\
\stackrel{n}{n}\end{array}$ & $\overrightarrow{\underline{a}}$ & $\begin{array}{l}\tilde{\omega} \\
\stackrel{\underline{n}}{n}\end{array}$ & 离 & $\frac{m}{\stackrel{n}{a}}$ & $\frac{n}{\sqrt[n]{n}}$ \\
\hline $\mathrm{SiO}_{2}$ & 52 & 47 & 37 & 0 & 0 & 0 & 0 & 0 & 0 \\
\hline $\mathrm{B}_{2} \mathrm{O}_{3}$ & 0 & 0 & 0 & 52 & 47 & 37 & 59 & 44 & 34 \\
\hline $\mathrm{CaCO}_{3}$ & 12 & 12 & 12 & 12 & 12 & 12 & 13 & 13 & 13 \\
\hline $\mathrm{P}_{2} \mathrm{O}_{5}$ & 6 & 6 & 6 & 6 & 6 & 6 & 3 & 3 & 3 \\
\hline $\mathrm{Na}_{2} \mathrm{CO}_{3}$ & 14 & 14 & 14 & 14 & 14 & 14 & 15 & 15 & 15 \\
\hline $\mathrm{ZnO}$ & 16 & 16 & 16 & 16 & 16 & 16 & 0 & 0 & 0 \\
\hline $\mathrm{TiO}_{2}$ & 0 & 5 & 15 & 0 & 5 & 15 & 10 & 10 & 10 \\
\hline $\mathrm{SrCO}_{3}$ & 0 & 0 & 0 & 0 & 0 & 0 & 0 & 15 & 25 \\
\hline Total & 100 & 100 & 100 & 100 & 100 & 100 & 100 & 100 & 100 \\
\hline
\end{tabular}




\subsection{Coating procedure}

Ti6Al4V plates $(88.9 \mathrm{~mm} \times 12.7 \mathrm{~mm} \times 3.2 \mathrm{~mm}$; McMaster-Carr, Elmhurst, Illinois, USA) were polished using 1200 grit sand paper and degreased using isopropanol alcohol. Sieved glass powder $(<20 \mu \mathrm{m})$ was mixed with ethanol (ratio of 5:1, ethanol to glass mass), in a standard $5 \mathrm{ml}$ syringe for approximately 60 seconds and then deposited on the centre of the Ti6Al4V plates which were put on a leveled table. The coated samples were left for an hour at room temperature and then heated in the furnace for $15 \mathrm{~min}$ at coating temperature, $T_{\text {coat }}$, chosen to be between the transition temperature $\left(T_{g}\right)$ and crystallization temperature $\left(T_{x}\right)$. Table 2.2 presents the coating temperatures for each glass composition.

Table 2.2. Glass transition, crystallization and coating temperatures for different glass compositions.

\begin{tabular}{|c|c|c|c|}
\hline Glass & $T_{g}\left({ }^{\circ} \mathrm{C}\right)$ & $T_{x}\left({ }^{\circ} \mathrm{C}\right)$ & $T_{\text {coat }}\left({ }^{\circ} \mathrm{C}\right)$ \\
\hline SRT0 & 619 & 735 & 650 \\
\hline SRT1 & 592 & 670 & 630 \\
\hline SRT3 & 610 & 705 & 640 \\
\hline BRT0 & 521 & 603 & 520 \\
\hline BRT1 & 530 & 625 & 550 \\
\hline BRT3 & 523 & 633 & 550 \\
\hline Ly-B0 & 500 & 677 & 560 \\
\hline Ly-B3 & 525 & 618 & 573 \\
\hline Ly-B5 & 550 & 649 & 615 \\
\hline
\end{tabular}

\subsection{Chapter summary}

The information covered here was included to avoid repetition in later chapters. The glass preparation and coating procedures described above were parts of the sample preparation for the experiments of Chapters 3 through 6. 
3. Quantitative evaluation of the mode I critical strain energy release rate of bioactive glasses onto Ti6Al4V substrates 
The work contained in this chapter is based on the peer-reviewed, published paper ${ }^{128}$ :

Matinmanesh, A., Rodriguez, O., Towler, M.R., Zalzal, P., Schemitsch, E.H. and Papini, M., 2016. Quantitative evaluation of the adhesion of bioactive glasses onto Ti6Al4V substrates. Materials \& Design, 97, pp.213-221.

\subsection{Introduction}

Most techniques for characterizing the adhesion of bioactive glass coatings to metals give only qualitative or semi-quantitative information and most existing indentation based fracture mechanics methodologies are prone to large errors ${ }^{67}$. The presence of potentially large residual stresses introduced during the high temperature glass coating process further complicates matters since it may significantly affect coating performance. This chapter presents a technique for measuring the mode I critical strain energy release rate of the system $\left(G_{I C}\right)$. The particular enamelling procedure that is presented requires the use of special bi-layer double cantilever beam (DCB) specimens for this purpose. The technique is inspired by fracture mechanics testing methodologies first developed in the assessment of environmentally degraded structural adhesive

joints $\left(\right.$ e.g. $\left.{ }^{108-113,129}\right)$. The residual stresses in the coating and their impact on the fracture toughness of the system are also analysed.

\subsection{Experiments}

\subsubsection{Glass preparation}

Glass preparation was done according to the procedure of Section 2.2. SRT0 (Section 2.2) was the only glass composition used in this chapter.

\subsubsection{X-ray diffraction (XRD)}

X-ray diffraction (XRD) was performed on SRT0 glass, analyzing over the range of $20^{\circ} \leq$ $2 \theta \leq 80^{\circ}$, with a step size of $0.05^{\circ}$ using a PANalytical X-ray diffractometer (PANalytical, QC, Canada). A CuKa anode was employed, with a generator voltage of $30 \mathrm{kV}$ and a tube current of 
$10 \mathrm{~mA}$. Crystalline phases were identified using the International Centre for Diffraction Data (ICDD) standard diffraction patterns.

\subsubsection{Particle size analysis (PSA)}

After grinding and sieving the glass, particle size analysis (PSA) was undertaken to determine the average particle size of the glass powder. PSA in the range of $2 \mu \mathrm{m}-60 \mu \mathrm{m}$ was performed on three powder samples of SRT0 glass using a Beckman Coulter Multisizer 4 Particle size analyzer (BeckmanCoulter, Fullerton, CA, USA). The results were analyzed by Multisizer 4 software, with means and standard deviations based on measurements of 30,000 particles per sample.

\subsubsection{Differential scanning calorimetry (DSC)}

A combined differential scanning calorimetry-thermogravimetric analyzer (DSC-TGA) (SDT 2960 Simultaneous DSC-TGA, TA Instruments, DE, USA) was used to measure the glass transition temperature $\left(T_{g}\right)$ and crystallization temperature $\left(T_{x}\right)$. A heating rate of $20{ }^{\circ} \mathrm{C} \mathrm{min}^{-1}$ was employed using an air atmosphere with alumina in a matched platinum crucible as a reference. Measurement was carried out every $6 \mathrm{~s}$ between $20^{\circ} \mathrm{C}$ and $850{ }^{\circ} \mathrm{C}$.

\subsubsection{Mechanical testing}

The double cantilever beam (DCB) is a practical and reliable fracture mechanics specimen that has been used for measuring the mode I fracture toughness of layered materials ${ }^{82}$. In traditional DCB specimens, the layer to be tested is sandwiched between two adherends. However, as discussed below, the enamelling technique used in the present study required the use of a modified bilayer DCB specimen in which the glass coating was applied to only one adherend, and a layer of epoxy was used to attach the coated adherend to an uncoated one. The overall range of coating thicknesses studied was $84 \mu \mathrm{m}$ - $407 \mu \mathrm{m}$. 


\subsubsection{Coating of Ti6Al4V plates}

Ti6A14V plates $(88.9 \mathrm{~mm} \times 12.7 \mathrm{~mm} \times 3.2 \mathrm{~mm})$ were polished using 1200 grit sand paper and degreased using isopropanol alcohol. The dimensions were chosen based on a uniformly scaled-down version of the specimen dimensions suggested by ASTM D3433-99 ${ }^{130}$, the standard for traditional single layer DCB specimens used for larger-scale adhesive joint testing. The roughness of the plates was measured according to ASME B46.1 ${ }^{131}$, using an optical non-contact profilometer (Microphotonics Inc, Nanovea ST400) by recording five $0.8 \mathrm{~mm}$ traces along the center line of the plates with scan step size of $0.1 \mu \mathrm{m}$. The roughness profiles were analysed after applying a Gaussian filter with a cut-off wavelength of $0.25 \mathrm{~mm}$ on the recorded profile (ASME B46.1 $\left.{ }^{131}\right)$.

The coating procedure was done as per Section 2.3. In order to create specimens with different ranges of coating thickness, mixtures containing $0.1 \mathrm{~g}, 0.3 \mathrm{~g}$ and $0.45 \mathrm{~g}$ of glass powder were created and deposited on the plates. The coating thickness was measured by using the optical profilometer to record three parallel traces $4 \mathrm{~mm}$ apart along the longitudinal axis of the substrate with a step size of $5 \mu \mathrm{m}$ both before and after enamelling the surface. In both cases, the waviness was removed by applying a Gaussian filter with a cut-off wavelength of $0.25 \mathrm{~mm}$ (ASME B46.1

$\left.{ }^{131}\right)$. The difference in the resulting profiles yielded three thickness profiles per specimen. Point by point averages and standard deviations for each thickness profile were calculated and averaged to obtain the overall average and standard deviation for each specimen. Three specimens in each of three thickness ranges $(84 \mu \mathrm{m}-93 \mu \mathrm{m}),(230 \mu \mathrm{m}-274 \mu \mathrm{m})$ and $(370 \mu \mathrm{m}-407 \mu \mathrm{m})$ were tested. The standard deviation of the thickness in a given specimen varied between $7-13 \mu \mathrm{m}, 15-\mu \mathrm{m}$ and 25 $32 \mu \mathrm{m}$, for the low, medium and large thickness ranges, respectively.

\subsubsection{Residual stress measurement}

The thermal residual stresses in the coating induced due to CTE mismatch while enamelling was assessed using the procedure developed by Yu et al. ${ }^{132}$, which is based on the measurement of the change in the curvature of coated specimens before and after heating. Using the optical profilometer, three parallel profiles $4 \mathrm{~mm}$ apart were measured using a step size of 5 $\mu \mathrm{m}$ along the longitudinal axis on the uncoated side of the Ti6Al4V plates (Section 3.2.5.1 ), before and after enameling. The waviness profile was extracted by applying a Gaussian filter with a cut- 
off wavelength of $0.25 \mathrm{~mm}$ (ASME B46.1 ${ }^{131}$ ), and the deformed profile was obtained by subtracting the profiles before and after enameling. According to ${ }^{132}$, the signed curvature, $k$, of each sample can be found from the maximum deflection of the Ti6Al4V plate $\delta_{R}$, and the halfchordal length $L$, as

$$
|k|=\frac{2 \delta_{R}}{\delta_{R}^{2}+L^{2}}
$$

and the maximum residual stress in the glass, i.e. at the coating/substrate interface, can be calculated as:

$$
\sigma_{c_{\text {in }}}=\frac{P_{c}}{w t_{c}}+\frac{M_{c} t_{c}}{2 I_{c}}
$$

where,

$$
P_{c}=2 \frac{M_{c}+M_{s}}{t_{s}+t_{c}} ; \quad M_{i}=k E_{i} I_{i}
$$

and $M, t, w, E$ and $I$ are respectively bending moment, thickness, specimen width, Young's modulus and second moment of area of the cross section. The subscripts $i=c$ or $s$ to refer to the coating or substrate respectively and $P_{c}$ is the axial force in the coating induced by residual stresses. The Young's modulus and Poisson's ratio of the Ti6Al4V substrate were taken as 110 GPa and 0.34 , respectively ${ }^{133}$, and the Young's modulus of the epoxy as $4 G P a^{134}$. It was assumed that the glass coating had a Young's modulus and Poisson's ratio of $35 \mathrm{GPa}^{135,136}$ and $0.26^{137}$, respectively, based on values from the literature for 45S5 bioactive glass, which had a similar composition to that presently used.

Although typical deflections were only on the order of $10 \mu \mathrm{m}$, the optical profilometer made it possible to measure deflections to within $+/-40 \mathrm{~nm}$, resulting in a maximum error in calculated $\sigma_{c_{\text {in }}}$ of less than $1 \%$.

\subsubsection{Bilayer DCB specimen}

Preliminary attempts at fabricating traditional single layer DCB specimens failed because the alcohol in the slurry of Section 3.2.5.1 evaporated during curing in the oven, significantly reducing the coating thickness. Due to this coating shrinkage, and despite the use of wire spacers 
between the plates, it proved impossible to obtain a relatively uniform coating thickness, and many parts of the specimen were left un-bonded. Therefore, a bilayer DCB specimen in which a relatively tough epoxy was used to adhere the coated Ti6Al4V substrate to an uncoated one (Figure 3.1) was used, inspired by the 'open-face' DCB specimen developed for accelerated environmental degradation studies of structural adhesive joints (e.g. $\left.{ }^{108-113,129}\right)$.

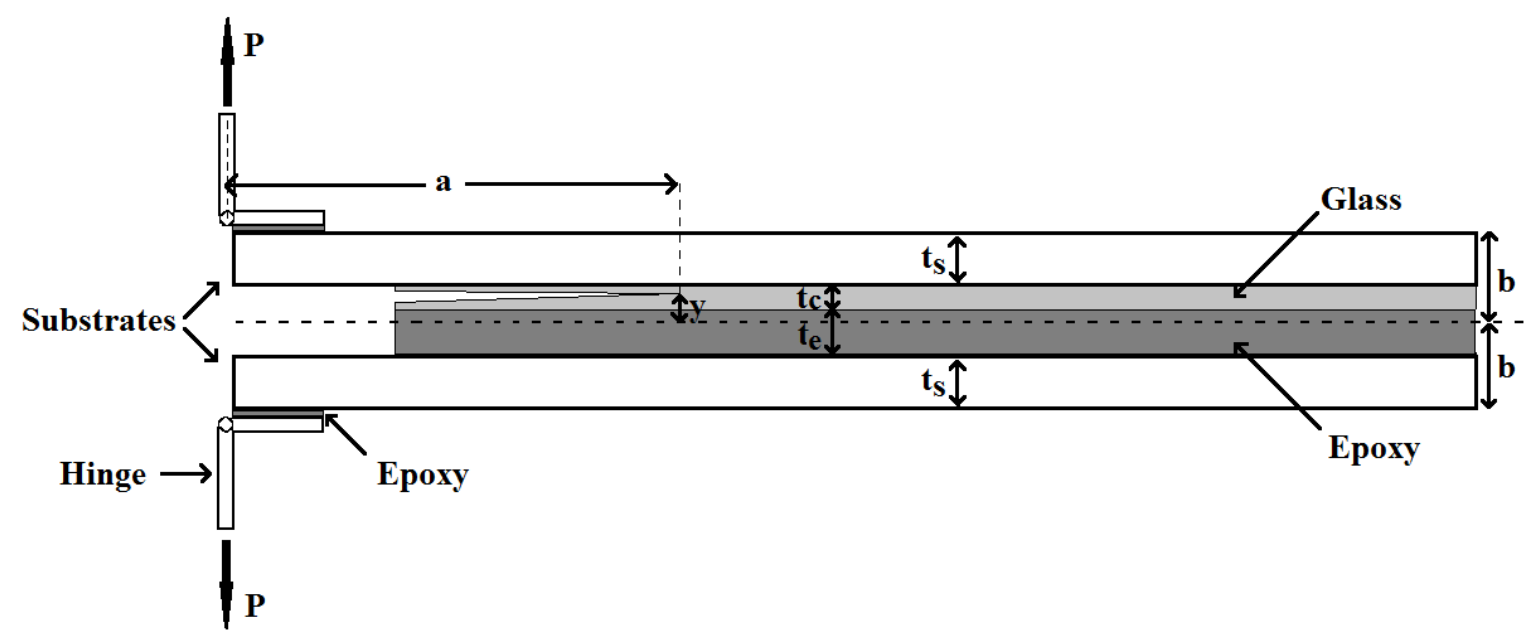

Figure 3.1. Double layer DCB specimen. Drawing is not to scale.

First, a thin layer of a commonly available room temperature cure epoxy (J-B Weld 8265S Cold Weld Compound, Sulphur Springs, TX, USA) was spread on the glass-coated adherends described in Section 3.2.5.1 using a small spatula. In order to protect the sides of the specimen so that the glass bondline remained visible during testing, and to control the thickness of the epoxy layer, $0.2 \mathrm{~mm}$ diameter wire was inserted around the periphery of the coated specimen. The wire was removed after approximately 10 minutes, when the epoxy set. Then the double sandwich specimen was clamped and left for 48 hours at room temperature to allow complete cure of the epoxy. Loading of the specimen was through two hinges (Figure 3.1) which were glued to the free ends of the specimen (one on each side) using the epoxy. In order to facilitate crack length measurement during testing, the bondline between the glass and substrate was covered with diluted typewriter correction fluid and the length of bondline was marked at fixed intervals.

\subsubsection{Specimen testing to determine $G_{I C}$}

The specimens were loaded according to established protocols for the determination of the mode I critical strain energy release rate, $G_{I C}$ in structural adhesive joint testing ${ }^{130,138}$. A United 
Universal Tester (STM series, United Testing Systems, Inc., Huntington Beach, CA, USA) with a $500 \mathrm{~N}$ load cell was used in displacement control to load the specimens by using friction grips on hinges with a crosshead displacement rate of $0.5 \mathrm{~mm} / \mathrm{min}$. A digital microscope camera (OptixCam Summit SK2-14X, Roanoke, VA USA) with a high magnification lens having a field of view of $3 \mathrm{~mm}$ was mounted on a motorized stage to allow the crack propagation to be monitored. The initial pre-crack length, i.e., the distance from line of action of the applied force to the crack tip, was recorded. The load was applied to the specimen until the crack was visually observed to propagate, also corresponding to a sudden drop in force. At this point, the displacement was held constant until the load levelled off at an approximately constant value and the crack stopped growing. The crack length was recorded, and the loading then restarted until the second crack propagation. By repeating this start and stop process, it was possible to obtain data for between 2 to 4 measurements of $G_{I C}$, the mode I critical strain energy release rate (amount of energy released in creating a unit area of crack extension) on each specimen. A total of 3 specimens were tested for each coating thickness range.

$G_{I C}$ for a thin coating layer between two identical adherends of thickness $t_{s}$ can be calculated as ${ }^{82}$

$$
G_{I C}=\frac{12 P_{\max }^{2} a^{2}}{\left[E_{s} w^{2} t_{s}^{3}\right]}
$$

where $P_{\max }$ is the load to propagate the crack, $w$ is the width of the substrate, and $a$ is the distance from crack tip to the hinge's axis of rotation (Figure 3.1). It is noted that Equation (3.4) neglects the effect of the compliance of the epoxy layer and coating. However, the error is small because the vast majority of the energy released due to crack extension is in the metal adherends. Applying the spring model of Troczynski and Camire ${ }^{139}$ of the present system, it was found that the error in $G_{I C}$ introduced by neglecting the compliance of the epoxy and glass layers was in all cases $<10 \%$. Further details are provided in Appendix A. 


\subsection{Results and discussion}

\subsubsection{X-ray diffraction (XRD)}

The presence of any crystal phases in SRT0 was evaluated using XRD, as shown in Figure 3.2. The trace was compared to the ICDD and the phase identified as Sodium Calcium Phosphate Silicate $\mathrm{Na}_{2} \mathrm{Ca}_{4}\left(\mathrm{PO}_{4}\right) \mathrm{SiO}_{4}$ (Ref. 00-033-1229).

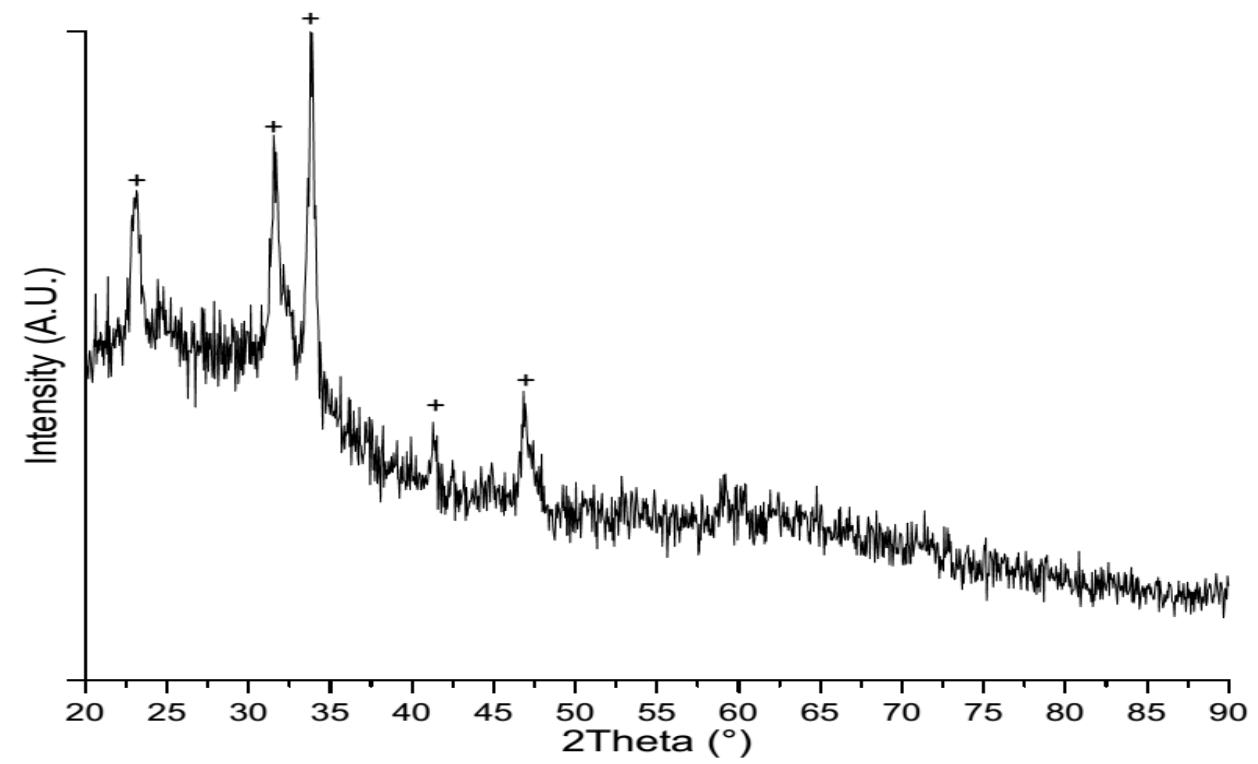

Figure 3.2. XRD pattern for SRT0. + indicates Sodium Calcium Phosphate Silicate $\mathrm{Na}_{2} \mathrm{Ca}_{4}\left(\mathrm{PO}_{4}\right) \mathrm{SiO}_{4}$ phase (Ref. 00-033-1229).

\subsubsection{Differential scanning calorimetry (DSC)}

The DSC trace for SRT0 is shown in Figure 3.3 yielding values of $T_{g}=619^{\circ} \mathrm{C}$, and $T_{x}=$ $735^{\circ} \mathrm{C}$. As mentioned in Section 3.2.5.1, the enameling temperature was chosen as $650^{\circ} \mathrm{C}$, ensuring it was greater than $T_{g}$, hence allowing for flow on the surface of the metal to be coated, but less than $T_{x}$ to avoid the promotion of further crystallization in the glass. 


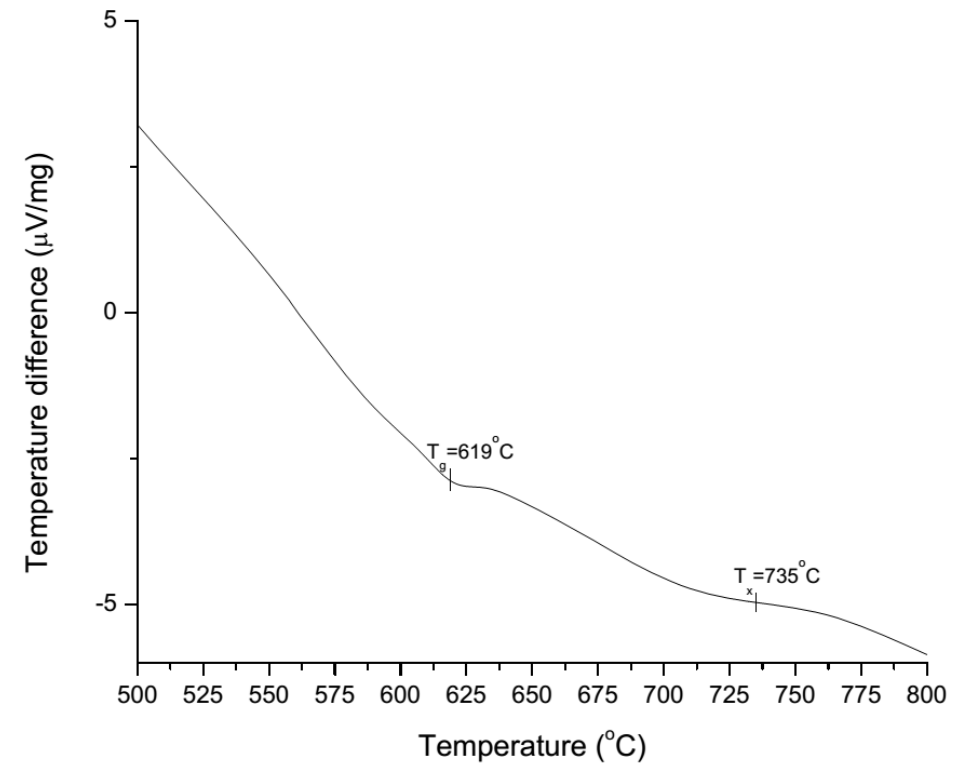

Figure 3.3. DSC trace of SRT0. Exo up.

\subsubsection{Particle size analysis}

The glass particle size after grinding and sieving was $5.3 \pm 4.0 \mu \mathrm{m}$. The D10, D50 and D90 values were found to be $2.1 \mu \mathrm{m}, 3.2 \mu \mathrm{m}$ and $9.8 \mu \mathrm{m}$, respectively. At least $50 \%$ of the glass particles had a diameter less than $3.2 \mu \mathrm{m}$, a favourable condition since small particle sizes result in better sintering ${ }^{140}$.

\subsubsection{Substrate roughness}

Since an aim was to investigate the effect of coating thickness on the fracture toughness of the system, it was important to ensure that the Ti6Al4V substrate roughness was relatively constant amongst the nine available plates. The average and standard deviation of the five measured roughness $R_{a}$ traces (Section 3.2.5.1) were in the range of 0.5 to $0.6 \mu \mathrm{m}$, and $0.02 \mu \mathrm{m}$ to $0.05 \mu \mathrm{m}$, respectively, for each of the nine plates. 


\subsubsection{Residual stress measurement}

As discussed in Section 5.2.3.2, the signed curvature, $k$, of the deformed profile of titanium substrates obtained by subtracting the measured back side profile of the substrate before coating from that after coating, was calculated using Equation (3.1), and the resulting residual stress using Equation (3.2).

Table 3.1 shows the mean and standard deviations for three traces on each specimen, and indicates that, as the coating thickness increased, the singed curvature of the sample, and the tensile residual stresses in the coating increased.

\subsubsection{Mode I critical strain energy release rate}

Because the glass was much more brittle than the epoxy and substrate, in all cases, the crack propagated within the glass, close to the interface between the glass and the substrate. Figure 3.4 shows typical force versus crosshead displacement and force versus crack length relationships obtained during bilayer DCB specimen testing. Initially, the applied force increased in an irregular fashion with the displacement while the slack in the load train was taken up (first ramp in Figure 3.4a). At the first crack propagation (first peak on both curves), the force decreased suddenly, and the crosshead advancement on the testing machine was stopped. The peak force and corresponding crack length were used for the first measurement of $G_{I C}$. The force dropped as the crack extended (Figure 3.4b) until both curves reached the first minimum. The crosshead displacement was then restarted and the force increased at a constant crack length until the second crack propagation occurred (second peak in Figure 3.4), after which the force suddenly dropped and the crosshead was stopped, etc. By recording the critical force and crack length measured from the hinge's axis of rotation for each crack propagation, multiple $G_{I C}$ values could be measured on a single specimen using Equation (3.4). 


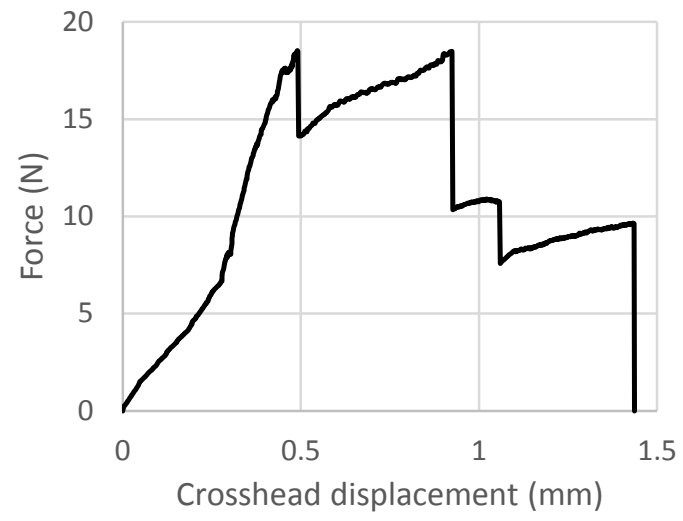

(a)

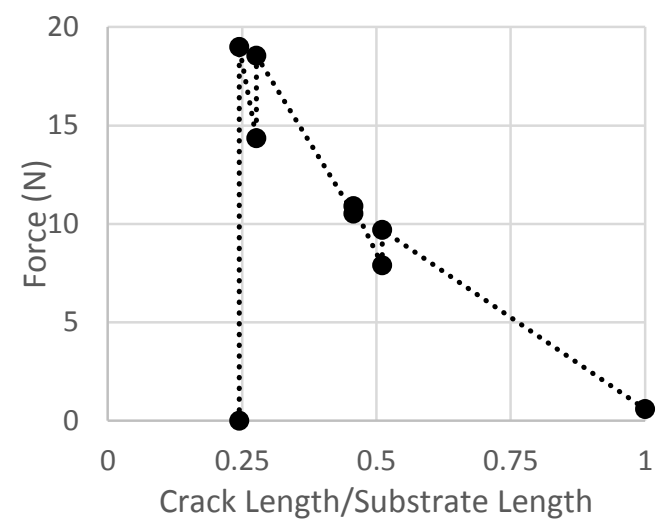

(b)

\section{Figure 3.4. Typical force vs. crosshead displacement and force versus normalized crack length}

curves obtained from DCB tests. The dashed line is to help guide the eye.

Figure 3.5, in which specimens with similar coating thickness are grouped, shows that the measured $G_{I C}$ values obtained from multiple measurements on three specimens are relatively constant, within a given thickness range. This indicates good repeatability in the proposed methodology.

In fracture testing of relatively tough layered materials, it is often found that there is an "Rcurve" behaviour in which the $G_{I C}$ values from the initial pre-crack are initially very low, but gradually increase to a steady state value after a number of crack propagations, as a toughened “damage zone" forms and grows to a steady state size ${ }^{64,83,84,110}$. As expected for a brittle glass ${ }^{82}$, Figure 3.5 shows that no such $R$ curve behaviour was found, i.e. the first measurement of $G_{I C}$ was generally no lower than subsequent ones, indicating that the damage zone was fully developed within the first crack propagation. There are other $G_{I C}$ measures for bioceramic and glass-ceramic coatings in the literature, obtained using a different method. For example Baino and VitaleBrovarone ${ }^{74}$ reported $G_{I C}=0.8 \mathrm{~J} / \mathrm{m}^{2}$, for a wollastonite-containing bioceramic coating on an aluminum substrate and Chen et al. ${ }^{75}$ reported $G_{I C}=0.065 \mathrm{~J} / \mathrm{m}^{2}$, for a glass-ceramic trabecularlike coatings to ceramic substrates. However since the coating-substrate systems used in this work is different than theirs, it is not surprising that the $G_{I C}$ values reported in Figure 3.5 are different than the ones found in these studies. 


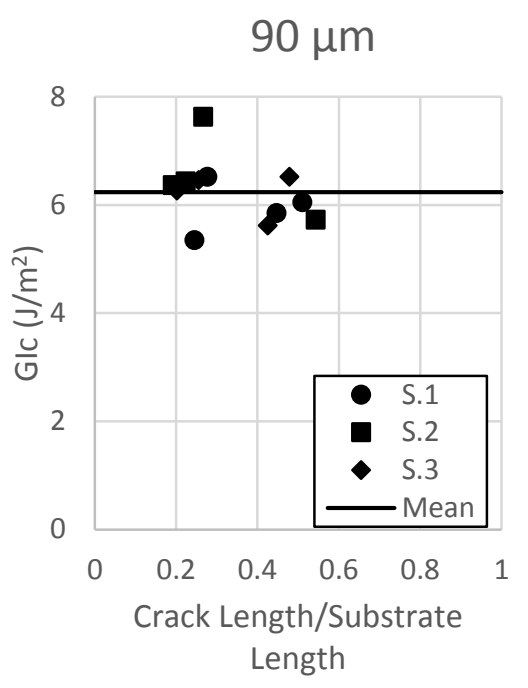

(a)

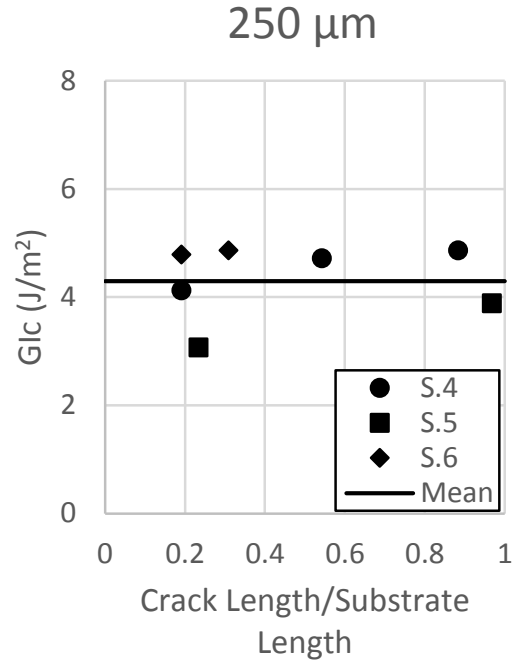

(b)

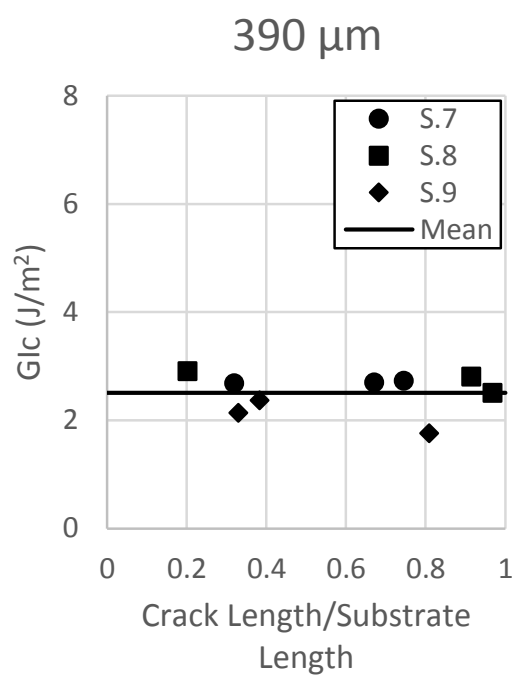

(c)

Figure 3.5. Critical strain energy release rate as function of normalized crack length for specimens having coating thickness (+/- standard deviation) of (a) $90 \pm 12 \mu \mathrm{m}$ (b) $250 \pm 22 \mu \mathrm{m}$ and (c) $390 \pm 32$ $\mu \mathrm{m}$. The mean values of $G_{I C}$, shown with horizontal lines, were $6.2 \mathrm{~J} / \mathrm{m} 2,4.3 \mathrm{~J} / \mathrm{m} 2$ and $2.5 \mathrm{~J} / \mathrm{m} 2$ for $90 \mu \mathrm{m}, 250 \mu \mathrm{m}$ and $390 \mu \mathrm{m}$ coating thicknesses, respectively.

\subsubsection{Crack path and mode ratio}

In general, cracking in layered materials can occur either in the layer itself or along the interface between the layer and the metal. Moreover, the critical strain energy release rate, $G_{c \text {, }}$ normally depends strongly on the mode ratio, a measure of the amount of opening (Mode I) versus shearing (Mode II) to which a planar crack is subjected ${ }^{141}$. In homogenous materials the resistance to crack propagation in Mode I, $G_{I C}$, is usually much lower than in mode II, $G_{I I c}$. In all of the presently tested samples, the crack was found to propagate within the coating close to the interface between the coating and the titanium alloy substrate, as shown in Figure 5.6. The average measured thickness of the remaining layer of the glass on the top arm of the substrate, $t_{r}$, is shown in Figure 3.6. The average measured thickness of the remaining layer of the glass on the top arm of the substrate, $t_{r}$, is shown in Table 3.1. 


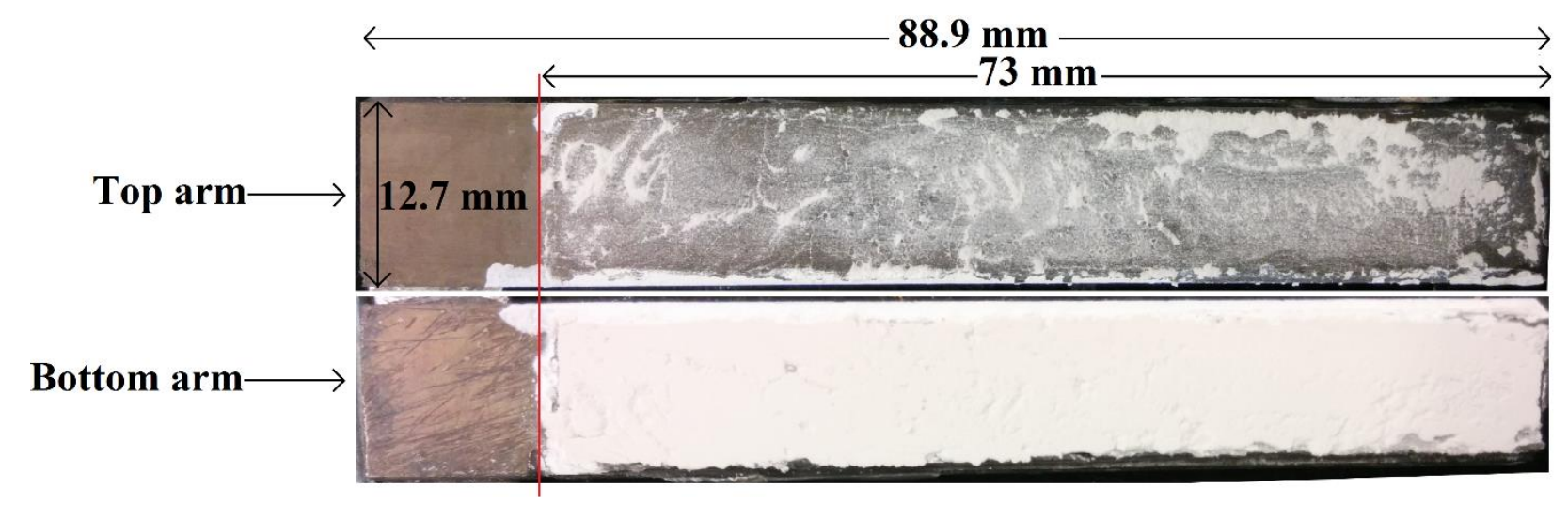

Figure 3.6. Image of fractured specimen. Bottom arm is the one with the epoxy layer below the thicker layer of residual glass.

The DCB test can be considered a pure mode I test only if the crack lies on the mid plane between the adherends which, because of the presence of the epoxy layer and the crack path described above, is not the case in the present work. It was therefore of interest to calculate the mode ratio or 'phase angle' of the loading, $\psi_{L}$, defined as ${ }^{141}$ :

$$
\psi_{L}=\arctan \sqrt{\frac{G_{I I}}{G_{I}}}
$$

where $G_{I}$ and $G_{I I}$ are respectively the mode I and mode II contributions to the strain energy release rate due to loading. Thus $\psi_{L}=0^{\circ}$ represents pure mode I, and $\psi_{L}=90^{\circ}$ represents pure mode II. Hutchinson and Suo ${ }^{141}$ suggest calculating $\psi_{L}$ in mixed-mode double cantilever beam specimens, based on offset ratio $y / b$, where $b$ is the half of the total thickness of the specimen $\left(b=\frac{2 t_{s}+t_{e}+t_{c}}{2}\right.$ ), $t_{e}$ is the thickness of the epoxy layer and $y$ is the offset of the crack plane with respects to the mid plane, i.e., $y=b-\left(t_{r}+t_{s}\right)$ (Figure 3.1). Even though the coating thickness varied, the offset ratio $y / b$ remained almost constant at $\sim 0.1$. Based on the phase angle-offset ratio graph, presented by Hutchinson and Suo ${ }^{141}$, the phase angle for all 9 samples was found as $\psi_{L} \sim 8^{\circ}$, i.e. the test was very near to pure mode I.

Table 3.1. Coating thickness $\mathrm{t}_{\mathrm{c}}$, signed curvature $k$, residual stresses $\sigma_{c_{i n}}$, and residual coating layer thickness $t_{r}$.

\begin{tabular}{|l|l|l|l|l|}
\hline Sample & $t_{c}(\mu \mathrm{m})$ & $k(1 / \mathrm{m}) \times 10^{-3}$ & $\boldsymbol{\sigma}_{\boldsymbol{c}_{\text {in }}}(\mathrm{MPa})$ & $t_{r}(\mu \mathrm{m})$ \\
\hline
\end{tabular}




\begin{tabular}{|c|c|c|c|c|}
\hline 1 & 84.0 & $2.0 \pm 0.3$ & $6.8 \pm 0.8$ & $21 \pm 9$ \\
\hline 2 & 87.7 & $2.0 \pm 0.2$ & $5.1 \pm 0.5$ & $15 \pm 4$ \\
\hline 3 & 92.3 & $2.8 \pm 0.4$ & $8.6 \pm 1.0$ & $23 \pm 11$ \\
\hline 4 & 230.7 & $9.7 \pm 1.8$ & $11.4 \pm 1.5$ & $62 \pm 41$ \\
\hline 5 & 252.3 & $11.1 \pm 1.0$ & $11.9 \pm 1.2$ & $95 \pm 79$ \\
\hline 6 & 273.7 & $13.6 \pm 2.0$ & $13.2 \pm 1.4$ & $103 \pm 62$ \\
\hline 7 & 370.0 & $19.5 \pm 2.5$ & $13.9 \pm 1.3$ & $115 \pm 91$ \\
\hline 8 & 382.3 & $21.4 \pm 1.7$ & $14.5 \pm 0.8$ & $105 \pm 87$ \\
\hline 9 & 408.0 & $28.3 \pm 2.2$ & $17.9 \pm 1.0$ & $123 \pm 98$ \\
\hline
\end{tabular}

\subsubsection{Dependence of $G_{I C}$ on coating thickness - effect of}

\section{residual stresses}

Figure 3.5 shows that the measured $G_{I C}$ value decreased as the coating thickness increased, i.e. the specimens made of thicker coatings were effectively less tough than those made of the thin ones. This trend is consistent with previous observations for glass coatings from the literature ${ }^{1,10}$ but has never been quantified; e.g. Gomez Vega et al. claimed that thinner glass coatings are significantly less prone to cracking or delamination ${ }^{1,10}$. One explanation for this trend is the effect of residual stresses on the measured $G_{I C}$ values. During testing, the residual stresses contribute to the release of the stored strain energy and therefore, significantly less energy from the applied loads will be required to reach the critical value for crack propagation. As described by Nairn ${ }^{142}$, such measurements that do not take into account residual stresses lead to an apparent $G_{I C}$ of the system, rather than the intrinsic toughness. Howard and Clyne ${ }^{143}$, used a numerical model of a 4 point bend specimen, and concluded that ignoring the effect of residual stresses in the case of a vacuum plasma sprayed coating can result in an apparent fracture toughness that is less than half of the intrinsic value. Therefore, the measured $G_{I C}$ values reported in Figure 3.5 are measures of 
apparent or 'global' fracture toughness of the system. . Nevertheless, for practical purposes, unless the residual stresses can somehow be eliminated, the global $G_{I C}$ values are the most appropriate for evaluating the actual performance of coatings since they can be used to calculate the applied external loads necessary to cause crack propagation. . Finally, it is noted that the residual stress can alter the local mode ratio at the crack tip ${ }^{143}$, so that $\psi_{L} \sim 8^{\circ}$ as reported in Section 5.3.5 represents the 'global' mode ratio based on external loading.

According to Hutchinson and Suo $1992^{141}$, the crack driving force (strain energy release rate) in the coating due to presence of residual tensile stresses $G_{R}$, can be calculated as follows:

$$
G_{R}=Z \frac{\sigma_{R}^{2} t_{c}}{E_{c}}
$$

where $t_{c}, E_{c}$ and $\sigma_{R}$ are the thickness, the Young's modulus and the residual stresses in the coating, respectively, and $Z$ is a unifying dimensionless 'driving force number' which depends on the cracking pattern and elastic mismatch between the coating and the substrate. Equation (3.6) shows that, for a given coating substrate system, the driving force increases both with coating thickness and residual stresses, which, as shown in Table 3.1, themselves increase with coating thickness. It can thus be concluded that thicker coatings result in a larger crack driving force due to residual stresses, i.e. a larger coating thickness results in a lower required external load to overcome the coating's resistance against cracking because the contribution of residual stresses to the total driving force is significantly higher. This conclusion is in agreement with Zhao et al. who also concluded that thick coatings show higher accumulation of residual stresses and consequently poorer adhesion ${ }^{94}$.

Crack driving forces due to residual stresses may be sufficiently large to overcome $G_{c}$, the critical strain energy release rate, even prior to applying an external load. The critical coating thickness, $t_{c r}$, in this case can be obtained by rearranging Equation (3.6) as ${ }^{141}$ :

$$
t_{c r}=\frac{G_{c} E_{c}}{Z \sigma_{R}^{2}}
$$

To determine this critical thickness for the present system, specimens were made with coating thicknesses higher than those in Table 3.1, and it was observed that cracking occurred 
under no external loading for coating thicknesses $>480 \mu \mathrm{m}$. As shown in Figure 3.7, the crack pattern was observed to be very similar to the "channelling" described in Ref. ${ }^{141}$.

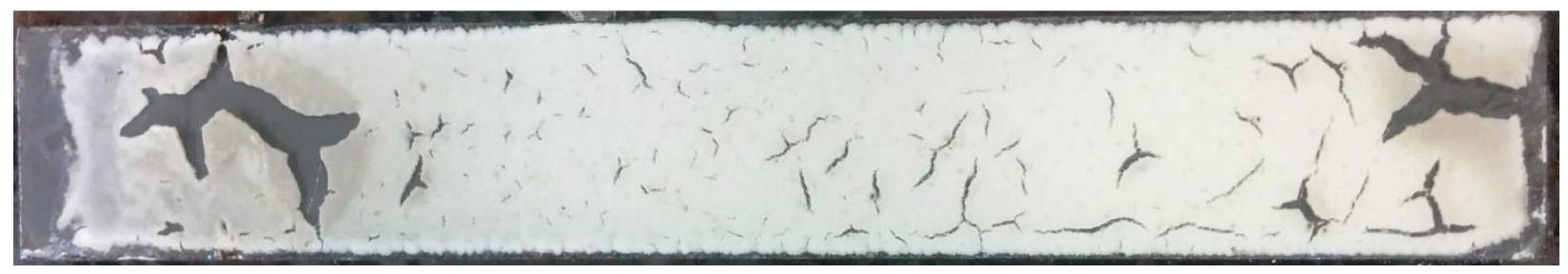

Figure 3.7. Typical crack pattern observed under zero external load for coating thicknesses greater than $480 \mu \mathrm{m}$. The coating thickness in this image is $540 \mu \mathrm{m}$

As mentioned above, the $Z$ value depends not only on the cracking pattern, but also the elastic mismatch between the titanium alloy substrate and glass coating, as expressed using the Dundurs parameters $\alpha$ and $\beta$ :

$$
\alpha=\frac{\mu_{S}\left(\kappa_{C}+1\right)-\mu_{c}\left(\kappa_{S}+1\right)}{\mu_{S}\left(\kappa_{c}+1\right)+\mu_{c}\left(\kappa_{S}+1\right)}, \quad \beta=\frac{\mu_{S}\left(\kappa_{C}-1\right)-\mu_{c}\left(\kappa_{S}-1\right)}{\mu_{S}\left(\kappa_{c}+1\right)+\mu_{c}\left(\kappa_{S}+1\right)}
$$

where the shear modulus, $\mu_{i}=E_{i} /\left[2\left(1+v_{i}\right)\right]$, and $\kappa_{i}=\left(3-v_{i}\right) /\left(1+v_{i}\right)$ for the plane stress condition and the subscripts $c$ and $s$ stand for coating and the substrate respectively. For the channelling crack pattern and the calculated value of $\alpha=0.53$ and $\beta=0.23$ for the present system, Hutchinson and Suo indicated that $Z=1.5^{141}$. The average residual stresses in the glass at glass/metal interface for 3 samples with an average coating thickness of $480 \mu \mathrm{m}$ was measured as $\sigma_{c_{\text {in }}}=23.4 \mp 1.1 \mathrm{MPa}$ using the methods of Section 5.2.3.2 . In Equation (3.7), $G_{c}$, the critical strain energy release rate of the bulk glass, based on the measurements of the next chapter (See the $G_{I C}$ of SRT0 in Figure 4.5) $G_{I c} \sim 9.2 \mathrm{~J} / \mathrm{m}^{2}$. Use of these $G_{c}, \sigma_{c_{i n}}$ and $Z$ values together with $E_{c}=35$ $G P a{ }^{135}$ in Equation (3.7), yields $t_{c r} \approx 490 \mu m$, which is in good agreement with the $480 \mu m$ value found experimentally. 


\subsubsection{T-stress}

In order to investigate the effect of the residual stresses in the coating on the direction stability of the crack within the coating, the 'T-stress' i.e. the tensile stress in the layer parallel to the crack plane, can be calculated for the system as ${ }^{144}$,

$$
T \approx \sigma_{R}+\left[\frac{a+1}{\alpha-1}\right]^{0.5} C_{1} K_{I c} t_{c}^{-0.5}
$$

where $\sigma_{R}$ is the residual stress in the coating, $K_{I C}$ is the mode I critical stress intensity factor that in plane stress condition can be converted to mode I critical strain energy release rate $G_{I C}\left(G_{I C}=\right.$ $\left.K_{I C} / E_{C}^{2}\right)^{82}$, and $t_{c}$ is the coating thickness. In Equation (3.9), $\alpha$ is one of the Dundurs parameters defined in Equation (3.8) and $C_{1}$ is an empirical function of the Dundurs parameters which can be found in Ref. ${ }^{141}$.

The necessary condition for straight cracking, i.e. the crack does not change its path and 'kink' during propagation, is that $T<0^{141,145-151}$. Using Equation (3.9) for the 9 specimens in Table 3.1 reveals that the T-stress for samples with $t_{c}<100 \mu \mathrm{m}$ is negative and for $t_{c}>100 \mu \mathrm{m}$ is positive. In other words, the crack plane in the thinner coatings is straight, but tends to destabilize and kink away from the interface for thicker coatings. This prediction is confirmed in Table 3.1 by the noticeably higher standard deviation in measured residual coating thickness seen at higher coating thicknesses.

\subsection{Chapter summary}

A novel methodology for quantifying the adhesive strength of enamelled bioactive glass coatings was presented, inspired in part by methods used in the fracture mechanics of structural adhesive joints. A method for applying a uniform thickness bioactive glass coating on Ti6Al4V substrate was developed and used with bi-layer double cantilever beam (DCB) specimens in order to measure the critical mode I interfacial or bioactive glass critical strain energy release rate $\left(G_{I C}\right)$. The technique, which allows multiple measurements of $G_{I C}$ on a single specimen, generated repeatable results, i.e. the measured values for specimens within a narrow range of coating thickness remained relatively constant. It was shown that the critical strain energy release rate of 
the coating decreased significantly with coating thickness, i.e., thinner glass coatings proved to have higher resistance against fracture than thicker ones. This was found to be due to differences in the residual stresses in the coating formed as a result of the thermal mismatch between the coating and the substrate.

Increases in the coating thickness were found to lead to higher residual stresses that tended to increase the crack driving force. If the coating thickness was sufficiently large, the residual stresses were sufficient to crack the glass layer, even prior to applying an external load. The critical thickness was calculated using existing fracture mechanics analyses, and found to be close to that measured experimentally, further confirming the efficacy of the fracture mechanics approach. Finally, the stability of the crack propagation within the coating was quantified by calculating the T-stress, and it was demonstrated that as the coating thickness increased, the crack destabilized and tended to kink rather than travel in a straight line. Overall, the present work in this chapter demonstrated that the methodologies show great promise in providing a more quantitative measure of bioactive glass coating adhesion than the more common scratch or indentation tests.

The mode I fracture mechanics testing methodology presented in this chapter will be used in Chapter 4 to investigate the effect of composition on the the fracture toughness of borate-based and silica-based coatings with different amounts of incorporated $\mathrm{TiO}_{2}$. 
4. Silica-Based and Borate-Based,

Titania-Containing Bioactive

Coatings Characterization: Mode

I Critical Strain Energy Release

Rate, Residual Stresses, Hardness,

and Thermal Expansion 
The work contained in this chapter is based on the peer-reviewed, published paper ${ }^{152}$ :

Rodriguez, O., Matinmanesh, A., Phull, S., Schemitsch, E.H., Zalzal, P., Clarkin, O.M., Papini, M. and Towler, M.R., 2016. Silica-Based and Borate-Based, Titania-Containing Bioactive Coatings Characterization: Critical Strain Energy Release Rate, Residual Stresses, Hardness, and Thermal Expansion. Journal of functional biomaterials, 7(4), p.32.

\subsection{Introduction}

In Chapter 3 a fracture mechanics testing methodology to quantify the glass/metal mode I critical strain energy release rate $\left(G_{I C}\right)$ was presented which proved to generate repeatable and consistent measures of $G_{I C}$ for the tested system. This testing methodology was applied on coatings with the thickness of 90 to $390 \mu \mathrm{m}$ to investigate the effect of glass coating's thickness on the $G_{I C}$ of the coating/substrate system and it was found that thinner glass coatings have higher resistance against fracture than thicker ones. Therefore, the work in this and the following chapters will only consider relatively thin coatings $(100 \mu \mathrm{m})$.

This chapter further characterizes the mechanics of bioactive glass coatings by studying the coating/substrate systems composed of two distinct glass series, SRT and BRT (Table 2.1). The former series is based on silica $\left(\mathrm{SiO}_{2}\right)$ and the latter is based on borate $\left(\mathrm{B}_{2} \mathrm{O}_{3}\right)$, with increasing amounts of titanium dioxide $\left(\mathrm{TiO}_{2}\right)$ incorporated at the expense of silica and borate, respectively. $\mathrm{B}_{2} \mathrm{O}_{3}$ has been shown to reduce the coefficient of thermal expansion (CTE) of glasses ${ }^{153}$, so that borate glasses have CTEs closer to that of the metallic substrate to be coated

(typically Ti6A14V, with a CTE of $9.5 \times 10^{-6} /{ }^{\circ} \mathrm{C}$ in the range of $0-315^{\circ} \mathrm{C}{ }^{154}$ ) compared to their $\mathrm{SiO}_{2}$ counterpart. Studying these two glass series will allow for the evaluation of the effect of $\mathrm{B}_{2} \mathrm{O}_{3}$ versus $\mathrm{SiO}_{2}$ on the resultant properties of the coating.

In this chapter the coefficient of thermal expansion (CTE) of the above mentioned glasses and its effect on the residual stresses post-coating will be studied. The Vickers indentation technique will be applied on the bulk glasses to determine the mode I fracture toughness and hardness of the bulk glass. Finally, the mode I critical strain energy release rate of the coating/substrate system will be determined using the technique developed in Chapter 3 . 


\subsection{Methods}

\subsubsection{Glass preparation}

Glass preparation was done based on the procedure outlined in Section 2.2. In this chapter, glass compositions from both SRT and BRT series at 0, 5 and $15 \mathrm{~mol} \%$ incorporated $\mathrm{TiO}_{2}$ (SRT0, SRT1, SRT3, BRT0, BRT1, BRT3) were studied.

\subsubsection{Discs preparation}

Approximately $200 \mathrm{mg}$ of each glass were pressed into a cylindrical mold with the diameter of $6 \mathrm{~mm}$ using a hydraulic press with pressure ranging between 2500-3000 psi; sample thicknesses were $2.84 \pm 0.19 \mathrm{~mm}$. The pressed discs were then heat treated to promote the coalescence of glass particles and create a sturdy solid to be used for CTE and hardness testing.

\subsubsection{Coating preparation}

The coating procedure was done as per Section 2.3 and a total of 5 coated specimens per glass composition were created.

\subsubsection{Coefficient of thermal expansion (CTE) measurement by linear dilatometry}

The CTE of each glass was tested based on current ASTM E228 "Standard Test Method for Linear Thermal Expansion of Solid Materials with a Push-Rod Dilatometer"155. Three glass disc samples per composition were prepared following the procedure outlined in Section 4.2.2, with samples measuring $6 \mathrm{~mm}$ in diameter and $12 \mathrm{~mm}$ in height (by stacking 4 discs), and tested with a Netzsch DIL 402 PC dilatometer (Netzsch Instruments, Burlington, MA, USA). A heating rate of $4^{\circ} \mathrm{C} / \mathrm{min}$ was employed, with testing temperature range from 25 to $300^{\circ} \mathrm{C}$ for the both glass series. Based on the measured lengths and temperature changes, CTE was determined as 


$$
\alpha_{m}=\frac{1}{\Delta T} \frac{\Delta l}{l_{0}}
$$

where $\alpha_{m}$ is the mean CTE of the glass, $\Delta T$ is the change in temperature with respect to the initial temperature, $l_{0}$ is the initial length of the test specimen, and $\Delta l$ is the change in length of the sample with respect to the initial length $l_{0}$.

\subsubsection{Residual stress and strain analysis}

Due to the mismatch between CTE of the substrate and of the coating, residual stresses at the interface are induced. To determine the residual stresses, Yu et al. ${ }^{132}$ proposed the use of EulerBernoulli beam theory on bi-layer materials with different CTEs subjected to thermal loading. The residual stress at the interface experienced by the glass coating is

$$
\sigma_{c_{i n}}=\frac{P\left(t_{s}+t_{c}\right)\left(\frac{t_{c}}{2}\right)}{2 I_{c}+\frac{2 E_{s} I_{s}}{E_{c}}}
$$

where

$$
P=\frac{\left(\alpha_{\text {Ti6Al4V }}-\alpha_{\text {glass }}\right)\left(T_{\text {coat }}-T_{i}\right)}{\frac{1}{E_{c} A_{c}}+\frac{1}{E_{s} A_{s}}+\frac{\left(t_{s}+t_{c}\right)^{2}}{4\left(E_{c} I_{c}+E_{s} I_{s}\right)}}
$$

and $\alpha_{\text {glass }}$ is the CTE of the glass, $\alpha_{T i 6 A l 4 V}$ is the CTE of the titanium substrate $\left(9.5 \times 10^{-6} /{ }^{\circ} \mathrm{C}\right), T_{\text {coat }}$ is the coating temperature (from Table 2.2$), T_{i}$ is the room temperature $\left(25^{\circ} \mathrm{C}\right), t_{s}$ is the thickness of the titanium substrate $(3.2 \mathrm{~mm}), t_{c}$ is the thickness of the glass coating $(90 \mu \mathrm{m}), I_{s}$ is the second moment of area of the titanium substrate, defined as $I_{s}=\frac{w t_{s}^{3}}{12}$, where $w$ is the width of the titanium substrate and of the glass coating $(12.7 \mathrm{~mm}), I_{c}$ is the second moment of area of the glass coating,

defined as $I_{c}=\frac{w t_{c}^{3}}{12}, E_{s}$ is the modulus of elasticity of the titanium alloy substrate $(110 \mathrm{GPa}), E_{c}$ is the modulus of elasticity of the glass coating $\left(35 \mathrm{GPa}^{136}\right), A_{s}$ is the cross-sectional area of the titanium substrate, defined as $A_{s}=w t_{s}$, and $A_{c}$ is the cross-sectional area of the glass coating, defined as $A_{c}=w t_{c}$. 


\subsubsection{Vickers hardness}

Three glass disc samples per composition were prepared for hardness testing were prepared as described in Section 4.2.2 . An HM-114 Mitutoyo Testing Machine (Mitutoyo, Mississauga, ON, Canada) was utilized, equipped with a Vickers indenter, and loading the samples with a force of $1 \mathrm{kgf}(9.81 \mathrm{~N})$ for $10 \mathrm{~s}$. The indent diagonals were measured through the integrated optical microscope at 20x.

\subsubsection{Mode I critical strain energy release rate of the bulk glass using Vickers indentation}

The mode I crtitcal strain energy release rate of the bulk glasses was measured by indenting glass discs (3 samples per glass composition), prepared as per Section 4.2.2 and indented similarly to the process described in 4.2.6. A schematic depiction of the indent is shown in Figure 4.1. According to Anstis et al. ${ }^{58}$, the indentation load needs to be large enough to create an indent pattern that is well-defined and cracks that are longer than the indent diameter $(2 r)$, yet shorter than one tenth of the thickness of the sample $(320 \mu \mathrm{m}$ in this case) to avoid interactions with the lower free surface of the specimen. The indentation load is considered too large if it breaks the sample or causes a chipping on the sample's surface ${ }^{58}$.

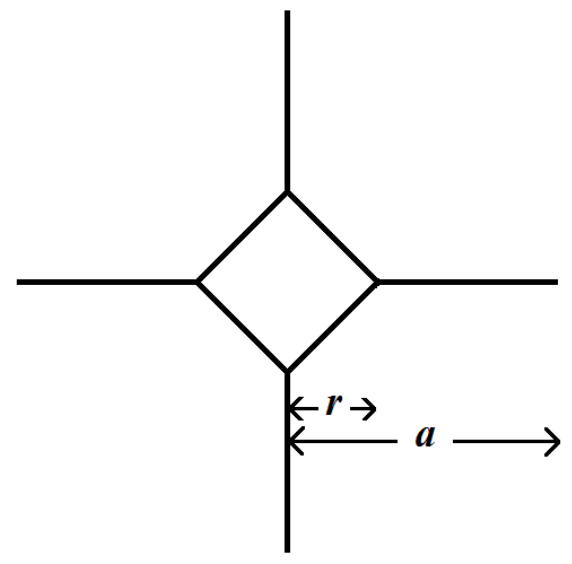

Figure 4.1. Schematic depiction of the cracks emanating from a Vickers indent. $r$ is the half of the diameter length of the dent, and $a$ is the crack length measured from the center of the indent. 
By trial and error, the appropriate indentation load to meet the aforementioned force criteria was found to be $3 \mathrm{kgf}(29.43 \mathrm{~N})$. This indentation load was applied using a Macro indenter (HM114 Mitutoyo Testing Machine, Mitutoyo, Mississauga, ON, Canada) normal to the surface of the glass for the duration of $10 \mathrm{~s}$. The length of the crack was measured through scanning electron microscope (SEM) using a JEOL JSM-6380LV SEM (JEOL, Peabody, MA, USA). The mode I critical strain energy release rate of the bulk glass was found using the equation below ${ }^{67,82}$ :

$$
G_{I C}=\frac{\alpha_{c a l i b}^{2} \times P_{i n}^{2}}{H a^{3}}
$$

where $P_{\text {in }}$ is the indentation load, $H$ is hardness of the glass (as measured per Section 4.2.6 ), $a$ is the length of the surface trace of the half penny crack measured from the center of the indent, and $\alpha_{\text {calib }}$ is the calibration constant $\alpha_{\text {calib }}=0.016 \pm 0.004{ }^{58}$. Equation (4.4) is derived for plane stress. Even though the current application does not completely satisfy the plane stress condition, Equation (4.4) can still be used as an approximation of $G_{I C}$ given that the thickness of the discs is less than half of their diameter.

\subsubsection{Coating/substrate system mode I strain energy release rate}

The mode I critical strain energy release rate $\left(G_{I C}\right)$ of the coating on the substrate was evaluated following the procedure outlined in Section 3.2.5.4. Three samples per glass composition were tested, with at least two measurements obtained per sample. Coated samples prepared as described in Section 4.2.3 were used to make the test specimens, then an epoxy layer (J-B Weld 8265-S Cold Weld Compound, Sulphur Springs, TX, USA) was deposited to cover the

glass and attach the second titanium alloy substrate. Specimens were loaded using a STM United Tensile Tester (United Testing Systems, Inc., Huntington Beach, CA, USA) using a 500-N load cell at a rate of $0.5 \mathrm{~mm} / \mathrm{min}$; then based on the recorded loads, the mode I critical strain energy release rate $G_{I C}$ was calculated as 


$$
G_{I C}=\frac{12 P_{\max }^{2} a^{2}}{E_{s} w^{2} t_{s}^{3}}
$$

where $P_{\max }$ refers to the load to start crack, $a$ is the crack length measured from the line of action of the force, $E_{s}$ to the tensile modulus of the substrate $(110 \mathrm{GPa}), w$ to the specimen width $(12.7$ $\mathrm{mm})$, and $t_{s}$ is the thickness of the substrate $(3.2 \mathrm{~mm})$.

\subsubsection{Statistical methods}

The results of all the measurements were expressed as means with experimental scatter expressed as a standard deviation. Additionally, one-way analysis of variance (ANOVA) was employed to analyze the data to determine significance in mean difference across the gathered data when $p<0.05$. Post-hoc Tukey test was used on MiniTab 17 (MiniTab Inc., State College, PA, USA). The Tukey test assumes equal variance in the data sets being analyzed to determine the significance in mean difference across all factors (i.e. all glasses in both series).

\subsection{Results and discussion}

\subsubsection{Coefficient of thermal expansion (CTE) measurement by linear dilatometry}

Results from the measurement of the CTE are plotted in Figure 4.2. The CTE of the silicabased glasses were found to be consistently greater than the CTE of Ti6Al4V, with the percentage difference ranging between $11.1 \%$ and $24.0 \%$, whereas the CTE for the borate-based glasses were found to be below the CTE of Ti6Al4V with smaller percentage difference, ranging between 4.1 and 5.8\%. In statistical terms, the CTE for all borate-based glasses were found to be equivalent; the CTE for SRT0 and SRT1 were also found to be equivalent.

The CTE results confirmed that the borate-based glasses possessed CTEs comparable to that of Ti6Al4V, evidenced by the reduced percentage difference of the CTEs between the boratebased glasses and Ti6Al4V, compared to the silica-based glasses. However, the CTE for the proposed silica-based glasses was significantly lower than what has been reported for other similar 
glasses (e.g. CTE of Bioglass ${ }^{\circledR} 45 \mathrm{~S} 5$ has been reported to be $15.1 \times 10^{-6} /{ }^{\circ} \mathrm{C}$ over the range from $200-400^{\circ} \mathrm{C}^{25}$ ), indicating that the proposed silicate formulations would provide better adhesion to the metallic substrate compared to other silica-based glasses since these novel silicate glasses would induce tensile residual stresses of lower magnitude when used as coatings compared to Bioglass ${ }^{\circledR} 45 \mathrm{~S} 5$. Hence, they would offer better resistance to crack growth. Since the addition of $\mathrm{TiO}_{2}$ increased CTE, then the control silicate formulation should be analyzed to understand the causes behind the reduced CTE. Compared to 45S5, SRT0 contains a higher molar percentage of $\mathrm{SiO}_{2}$ and contains $\mathrm{ZnO}$ at $16 \mathrm{~mol} \%$, whereas $45 \mathrm{~S} 5$ does not include $\mathrm{ZnO}$. Higher $\mathrm{SiO}_{2}$ content is known to decrease CTE of silica-based glasses ${ }^{42}$, which explains how substituting $\mathrm{SiO}_{2}$ for $\mathrm{TiO}_{2}$ in the SRT glasses translated into an increase in the CTE. Furthermore, ZnO increase (or inclusion, in this case) has been proven to decrease the CTE of silica-based glasses ${ }^{156}$, explaining the reduced CTE of SRT0 compared to $45 \mathrm{~S} 5$.

Since the CTEs of the borate-based glasses are lower than that of the substrate, the difference in shrinkage caused the glass coating to experience compressive residual stresses. Compressive residual stresses are beneficial, acting to prevent cracks from propagating, thus requiring higher stresses to cause coating failure ${ }^{157-159}$. On the other hand, the CTE of the silicabased glasses induced tensile residual stresses, promoting the growth of cracks under loading 141,157. 


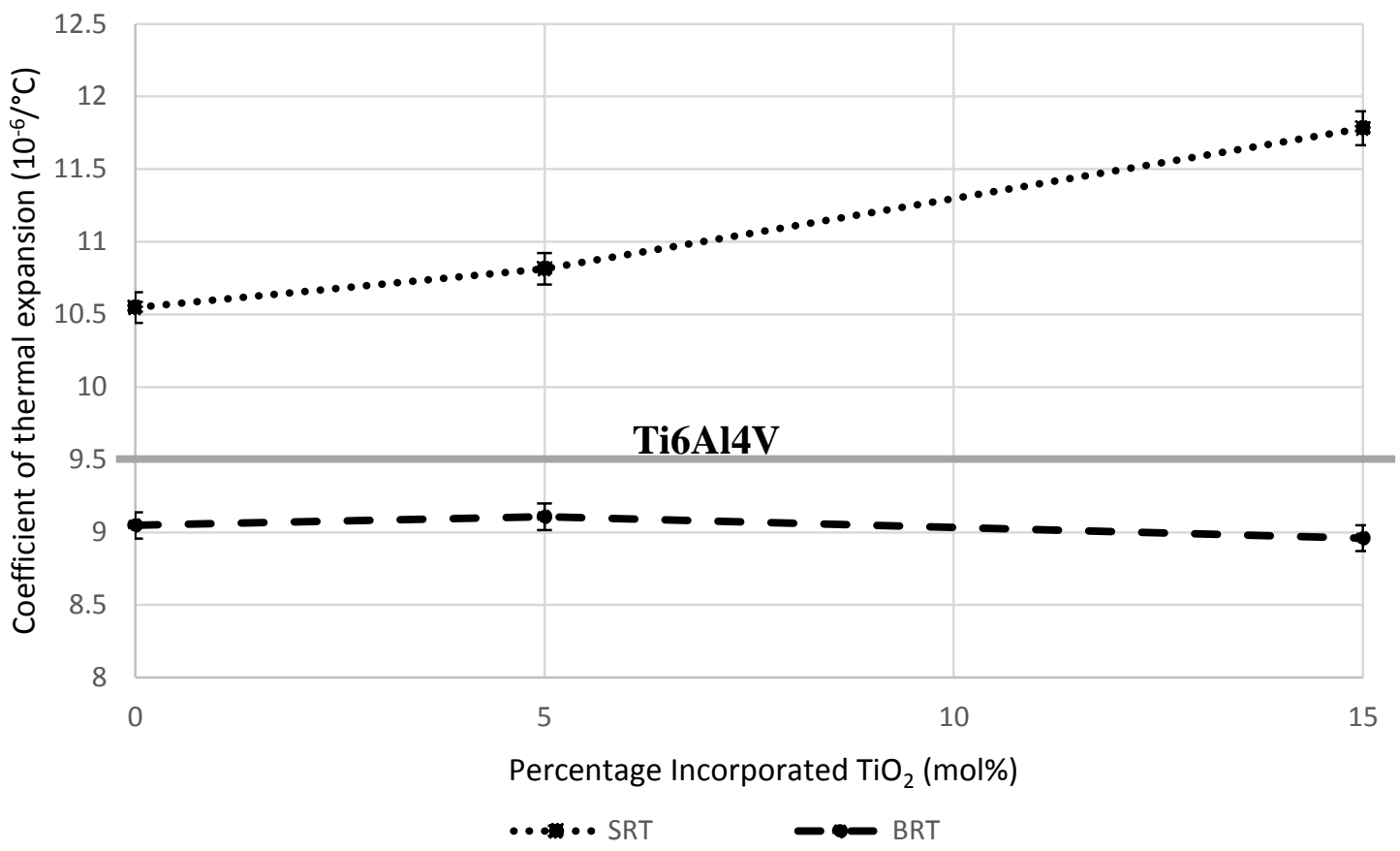

Figure 4.2. CTE for the SRT and BRT glasses, plotted along with the CTE of Ti6Al4V as reference $(n=3)$. Scatter bars indicate one standard deviation from the mean. The dashed lines are to help guide the eye.

\subsubsection{Residual stress analysis}

Residual stresses results are shown in Figure 4.3, where it can be seen that, in terms of the magnitude, greater stresses were experienced when the silica-based coatings were employed, compared to the borate-based ones. This is due to higher CTE mismatch between the silica-based glasses and the titanium substrate, especially for the case of SRT3 (15 mol\% incorporated $\mathrm{TiO}_{2}$ ). Borate-based glasses exhibit compressive residual stresses and silica-based glasses exhibit tensile residual stresses. 


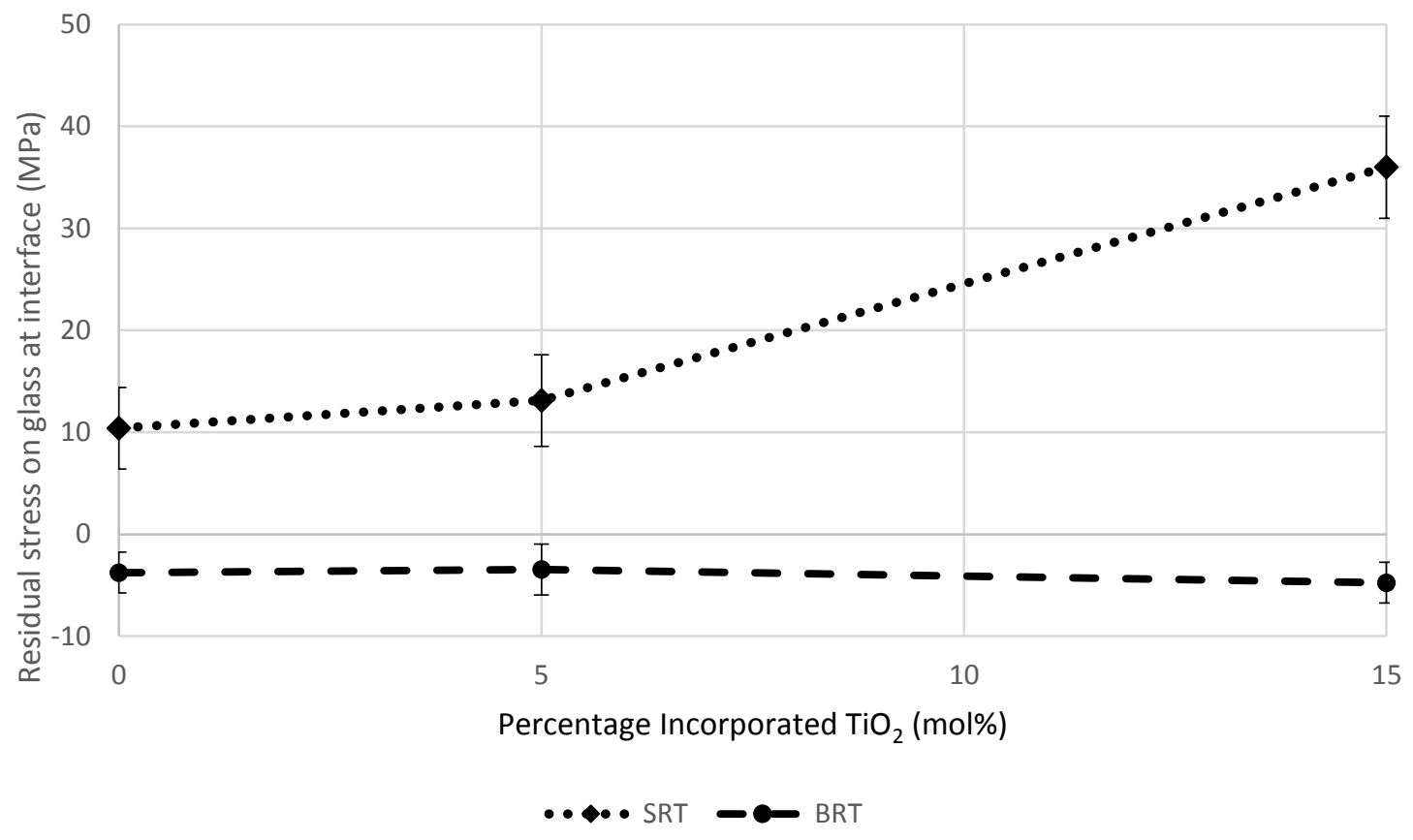

Figure 4.3. Residual stresses experienced in the glass coating at the coating/substrate interface using the SRT and BRT glasses as coating $(n=3)$. Scatter bars indicate one standard deviation from the mean. The dashed lines are to help guide the eye.

The residual stress in the SRT0 coating using the measured values of the CTE was estimated to be $9.6 \pm 2.4 \mathrm{MPa}$. This is consistent (no significant difference, $p<0.05$ ) with the 8.6 \pm 1.0 MPa value found in Section 3.3.6 for the same SRT0 coating/substrate system using measurements of the signed curvature of the coated assembly.

\subsubsection{Vickers hardness}

A substitution of $\mathrm{SiO}_{2}$ for $\mathrm{B}_{2} \mathrm{O}_{3}$ resulted in an increase in the Vickers hardness of the glass, as shown in Figure 4.4, statistically significant at 5 and $15 \mathrm{~mol} \%$ of incorporated $\mathrm{TiO}_{2}$. The incorporation of $\mathrm{TiO}_{2}$, however, did not significantly affect the hardness for the BRT glass series; for the SRT glass series, the addition of $\mathrm{TiO}_{2}$ to $5 \mathrm{~mol} \%$ decreased the hardness, but further addition did not significantly decrease it. 


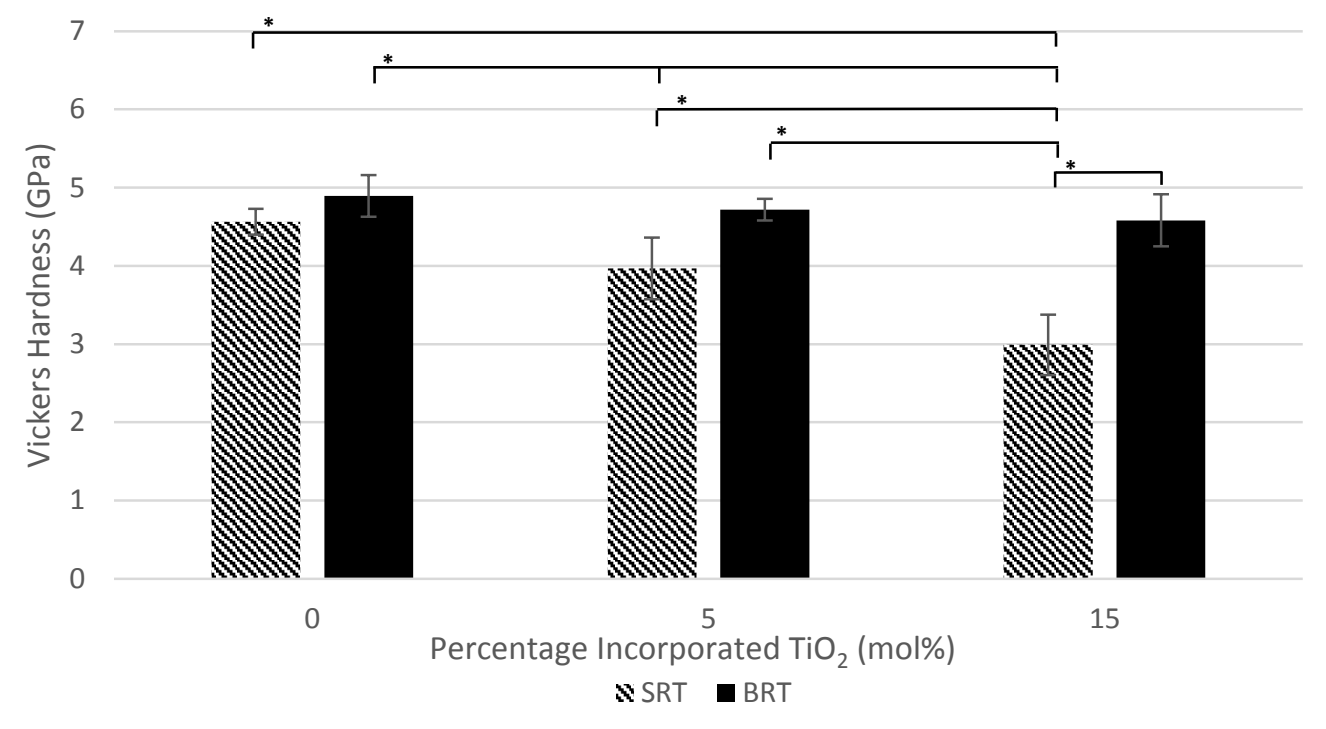

Figure 4.4. Vickers hardness for the SRT and BRT glasses $(n=3)$. Scatter bars indicate one standard deviation from the mean. Stars and bars show statistical significance $(p<0.05)$.

The bulk hardness of the silica-based glass series decreased as the amount of $\mathrm{TiO}_{2}$ incorporated into the glasses increased, while, in statistical terms, the hardness of the borate-based glasses did not significantly change $(p<0.05)$ with the addition of $\mathrm{TiO}_{2}$. The hardness of the borate-based glasses however, was significantly higher than the silica-based equivalent glasses at 5 and $15 \mathrm{~mol} \%$ incorporated $\mathrm{TiO}_{2}$.

\subsubsection{Mode I critical strain energy release rate of bulk glass using Vickers indentation}

The Vickers indentation test was performed to measure the bulk mode I critical strain energy release rate Equation (4.4) and the results are shown in Figure 4.5. A sample of the SEM image for SRT0 showing the indent and the cracks emanating from it is presented in Figure 4.6. Figure 4.5 also presents as reference points the data obtained from the literature for the mode I critical strain energy release rate of fused silica-based glass $\left(99.995 \% \mathrm{SiO}_{2}\right)$ and Pyrex (heat resistant borosilicate glass). Based on previous studies, the fracture toughness $K_{I C}$ and modulus of elasticity $E_{c}$ of fused silica glass and Pyrex are $0.80 \mathrm{MPa}^{1 / 2}{ }^{160}, 0.63 \mathrm{MPa}^{1 / 2} \mathrm{~m}^{161}$ and $72 \mathrm{GPa}$ ${ }^{160}, 67 \mathrm{GPa}{ }^{162}$, respectively. The following equation ${ }^{82}$, valid for plane stress condition, was used 
to convert these $K_{I C}$ and $E_{c}$ values to the $G_{I C}$ in Figure 4.5 , yielding $8.9 \mathrm{~J} / \mathrm{m}^{2}$ for fused silica and $5.9 \mathrm{~J} / \mathrm{m}^{2}$ for Pyrex.

$$
G_{I C}=\frac{K_{I C}^{2}}{E_{C}}
$$

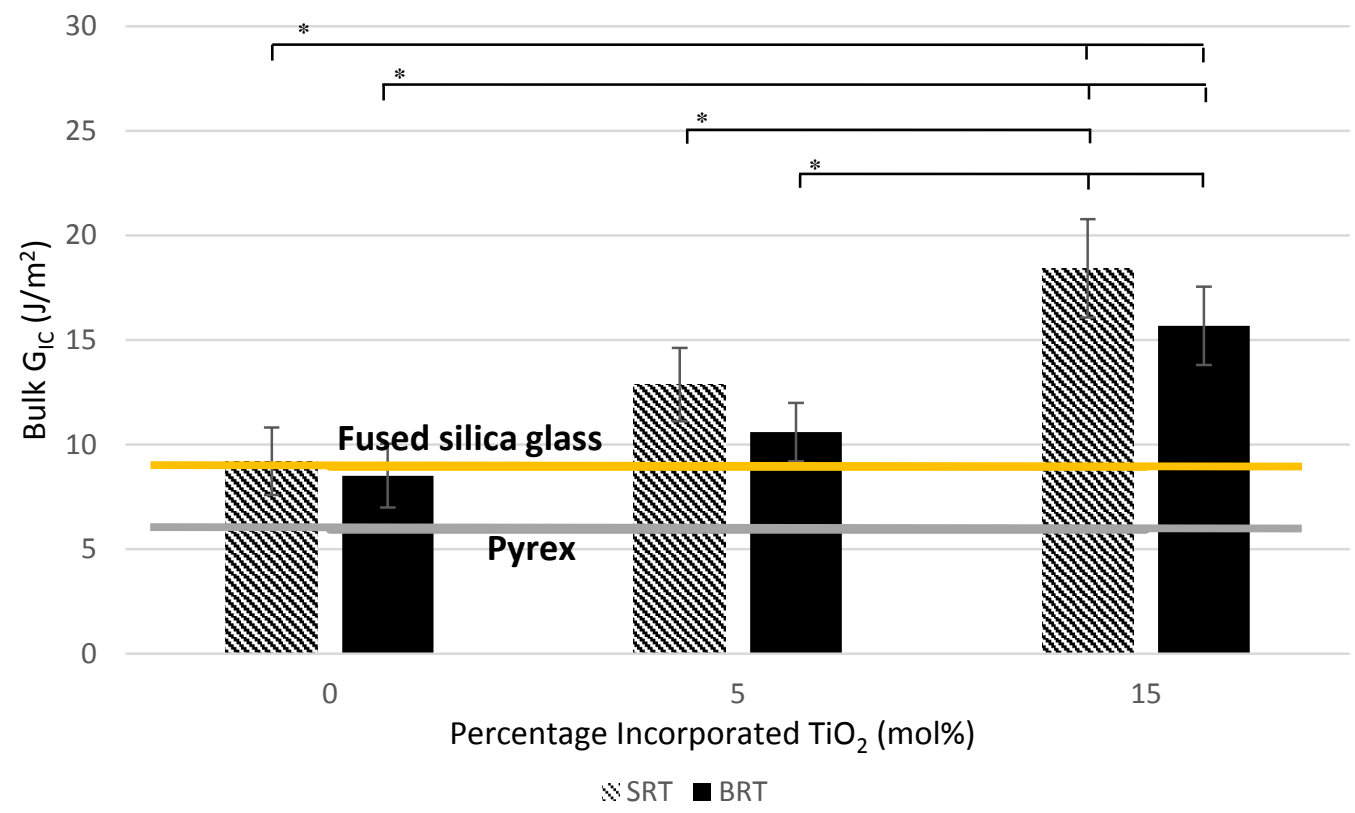

Figure 4.5. Bulk mode I critical strain energy release rates for the SRT and BRT glasses $(n=3)$. The $G_{I C}$ values for Fused silica glass and Pyrex obtained from the literature ${ }^{160,161}$ are also shown for reference. Scatter bars indicate one standard deviation from the mean. Stars and bars show statistical significance $(p<0.05)$.

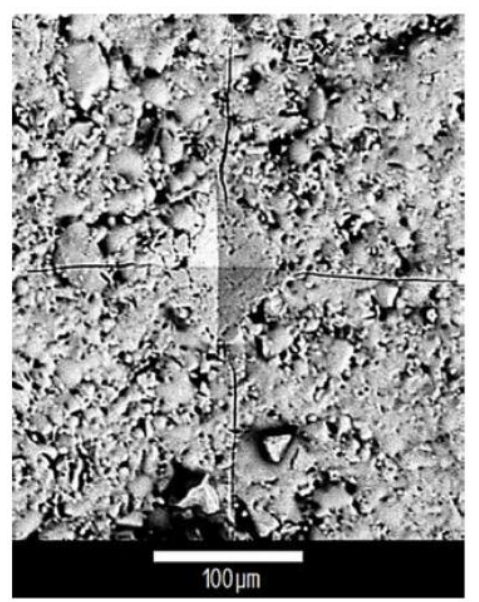

Figure 4.6. SEM of a Vickers indent on SRT0 with the emanating cracks. The average half diameter and crack length are $54.8 \mu \mathrm{m}$ and $187.9 \mu \mathrm{m}$, respectively. 
As shown in Figure 4.5, the measured bulk $G_{I C}$ of the control glass of the silica-based series, SRT0, was found to be comparable to that of fused silica glass. However, as the percentage of $\mathrm{TiO}_{2}$ in the silica-based glass series was increased, the mean $G_{I C}$ value increased, with significant differences $(p<0.05)$ observed between the SRT0 and SRT3 $(15 \mathrm{~mol} \%$ incorporated $\mathrm{TiO}_{2}$ ). There is limited literature available on the mode I critical strain energy release rate (or fracture toughness) of bioactive glasses, specifically on the effect of the inclusion of $\mathrm{TiO}_{2}$ at the expense of the backbone component. The authors hypothesize that the observed increase in the bulk $G_{I C}$ as the amount of $\mathrm{TiO}_{2}$ increased can be attributed to the presence of $\mathrm{Ti}^{4+}$ ions. Incorporation of such ions have been shown to strengthen glass systems and to improve their

mechanical properties ${ }^{163}$, due to their small ionic radius and high electrical charge ${ }^{164}$ that tends to strengthen the bonds in the glass. The bulk $G_{I C}$ of the control glass in the borate series, BRT0, was found not to be significantly different $(p<0.05)$ from that of SRT0; however, the bulk $G_{I C}$ of the remaining borate-based glasses were lower than their counterpart in the silica-based series. This is not surprising, as previous studies on boro-silicate glasses have shown that the incorporation of $\mathrm{B}_{2} \mathrm{O}_{3}$ at the expense of $\mathrm{SiO}_{2}$ can decrease the fracture toughness (directly related to $G_{I C}$ through Equation (4.5)) ${ }^{165,166}$. Furthermore, similar to silica-based series, the incorporation of $\mathrm{TiO}_{2}$ in the borate series glasses increased the fracture toughness.

\subsubsection{Mode I critical strain energy release rate of the coating/substrate system}

The mode I critical strain energy release rates for the coating/substrate system for both glass series are shown in Figure 4.7. Systems made with borate-based glasses exhibited higher critical strain energy release rates in mode I opposed to the silica-based coatings, with the exception of SRT1 and BRT1 (5 mol\% incorporated $\mathrm{TiO}_{2}$ ), which were statistically equivalent. As a function of percentage of $\mathrm{TiO}_{2}$ incorporated, for the silica-based series, there is no significant difference in the critical strain energy release rate between 0 and $5 \mathrm{~mol} \%$ incorporated $\mathrm{TiO}_{2}$ and between 5 and $15 \mathrm{~mol} \%$ incorporated $\mathrm{TiO}_{2}$; however, statistical difference is observed between 0 and $15 \mathrm{~mol} \%$ incorporated $\mathrm{TiO}_{2}$. Similarly, for systems made with the borate-based series, there is no significant increase $(p<0.05)$ in critical strain energy release rate between 0 and 5 mol\% 
incorporated $\mathrm{TiO}_{2}$, whereas a significant increase is found between 0 and $15 \mathrm{~mol} \%$ incorporated $\mathrm{TiO}_{2}$, and between 5 and $15 \mathrm{~mol} \%$ incorporated $\mathrm{TiO}_{2}$.

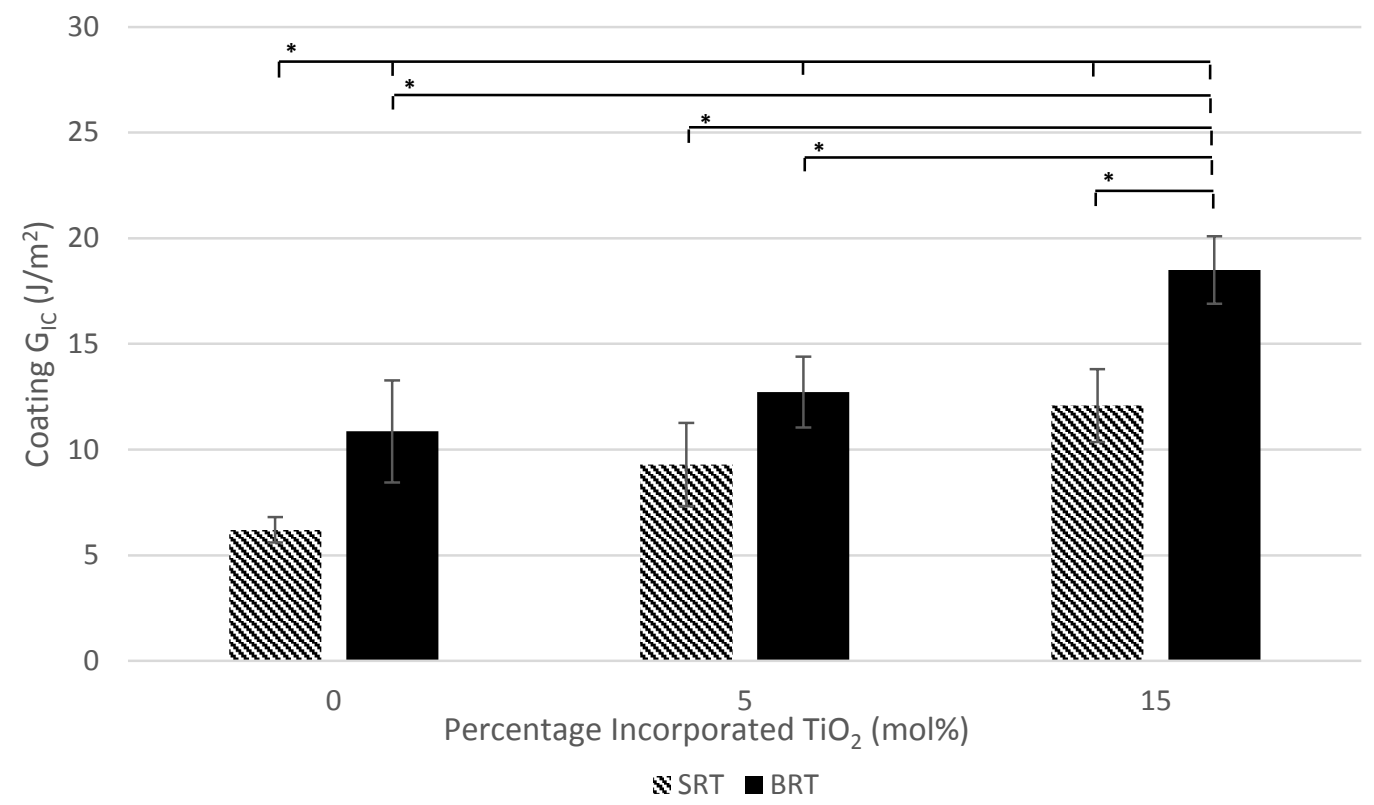

Figure 4.7. Mode I critical strain energy release rates for the coating/substrate systems with SRT and BRT glasses ( 3 samples per glass). Scatter bars indicate one standard deviation from the mean. Stars and bars show statistical significance $(p<0.05)$.

In terms of the $G_{I C}$ of the coating/substrate system, the results of Section 3.3.6 of previous chapter found the $G_{I C}$ for SRT0-based systems to be at $6.20 \pm 0.60 \mathrm{~J} / \mathrm{m}^{2}$. The work in this chapter expanded on these findings, determining that an increase in $\mathrm{TiO}_{2}$ content resulted in an increase in critical strain energy release rate, peaking at $12.08 \pm 1.72 \mathrm{~J} / \mathrm{m}^{2}$ for the silica-based coatings, and $18.50 \pm 1.60 \mathrm{~J} / \mathrm{m}^{2}$ for the borate-based coatings. This work supports the previous findings that titanium in the glass coating enhances chemical bonding to the titanium substrate ${ }^{153}$, resulting in a larger measured $G_{I C}$ for the SRT3 and BRT3 systems, both with $15 \mathrm{~mol} \%$ of $\mathrm{TiO}_{2}$ incorporated. Additionally, the trends of the $G_{I C}$ for the bulk glass and the coating/substrate system are similar, i.e., an increase in incorporated $\mathrm{TiO}_{2}$ translated into an increase in $G_{I C}$. This is similar to the work of Li et al. ${ }^{167}$, who studied the effect of the incorporation of strontium oxide into borate-based glass coatings applied to Ti6Al4V substrates, and found that $G_{I C}$ increased as the amount of strontium oxide increased.

Comparing the presented $G_{I C}$ values in Figure 4.5 with those in Figure 4.7 revealed that the glasses in the silica-based series have higher critical strain energy release rates in bulk form compared to when applied to the coating/substrate. On the contrary, in the borate-based series, the 
$G_{I C}$ was higher for the coating/substrate system. This effect may be attributed to the nature of the residual stresses that are created during the coating process, i.e., tensile in silica-based systems and compressive in borate-based systems (Section 4.3.2), with compressive residual stresses providing additional resistance to crack growth.

\subsection{Chapter summary}

This chapter further characterized the mechanics of bioactive glass coatings by synthesizing and characterizing silica-based and borate-based glasses in terms of their mechanical properties relevant to their use as metallic coating materials. It was observed that borate-based glasses exhibited CTE that were closer to the substrate's (Ti6A14V) CTE, a common alloy used in medical implants; this translated into higher mode I critical energy release rates for the boratebased glasses and lower residual stresses and strains at the coating/substrate interface, outperforming the silica-based glasses counterpart. An increase in the content of $\mathrm{TiO}_{2}$ in the glasses resulted in an increase in the mode I critical energy release rate for both the bulk glass and for the coating/substrate system and a decrease in the bulk hardness. Borate-based glass BRT3, with $15 \mathrm{~mol} \% \mathrm{TiO}_{2}$ incorporated, exhibited superior properties overall compared to the other proposed glasses in this work, as well as Bioglass® 45S5 and Pyrex. Due to this advantage of borate-based glasses, the work in Chapter 5 and Chapter 6 will only consider the borate-based glasses.

In the present chapter, the fracture toughness of the coating/substrate systems were evaluated in nearly mode I loading. Chapter 5 will present a methodology to measure the critical strain energy release rate under nearly pure mode II conditions of a series of borate-based glass coating/Ti6Al4V alloy substrate systems. 
5. Quantifying the mode II critical strain energy release rate of

borate bioactive glass coatings on Ti6Al4V substrates 
The work contained in this chapter is based on the peer-reviewed, published paper $^{168}$ :

Matinmanesh, A., Li, Y., Clarkin, O., Zalzal, P., Schemitsch, E.H., Towler, M.R. and Papini, M., 2017. Quantifying the mode II critical strain energy release rate of borate bioactive glass coatings on Ti6Al4V substrates. Journal of the Mechanical Behavior of Biomedical Materials, $75,212-221$.

\subsection{Introduction}

In Chapter 4 the effect of glass composition on the mechanical properties of the coating/substrate system was evaluated and since borate-based glass series exhibited superior mechanical properties overall compared to their silicate counterparts, the work in this and next chapters only considers the borate-based glasses.

The fracture mechanics testing methodology of Chapter 3 determined the critical strain energy release rate of glass/coating systems under mode I (opening) loading conditions, whereas the coatings on biomedical implants such as hip and knee prostheses are usually subjected to a combination of both shearing (mode II) and opening (mode I) loads ${ }^{36}$.

Even though the contribution of the shear loads on the implant can be significant in some cases, there appears to be no existing methodologies to quantify the critical strain energy release rate of bioactive glass coatings on metallic substrates under mode II conditions. In this chapter, the critical strain energy release rate under nearly pure mode II conditions of a series of boratebased glass coating/Ti6Al4V alloy substrate system was determined using the developed fracture mechanics testing methodology of Chapter 3 inspired by work done to assess environmentally degraded structural adhesive joints ${ }^{109-111}$. The technique allowed determination of the effect of the glass composition and mode ratio on the critical strain energy release rate of the coating/substrate system. The magnitude and distribution of residual stresses in the system were also determined, and their impact on the glass/metal fracture toughness analysed. 


\subsection{Experiments}

\subsubsection{Glass preparation}

As indicated above, borate-based bioactive glasses were studied as potential coatings for Ti6Al4V implants since they can be tailored to have a comparable CTE to Ti alloys ${ }^{5}$. In this chapter, three glasses from the Ly-B series with $0,15 \mathrm{~mol} \%$ and $25 \mathrm{~mol}_{\%} \mathrm{SrCO}_{3}$ (Ly-B0, Ly-B3 and Ly-B5, respectively) were prepared based on the procedure outlined in Section 2.3.

\subsubsection{Measurement of coefficient of thermal expansion}

The coefficients of thermal expansion (CTE) of the glasses were measured using linear dilatometry based on ASTM E228 ${ }^{155}$. For this purpose, $4 \pm 0.25 \mathrm{~mm}$ thick glass disc samples were created by pressing $0.4 \mathrm{~g}$ of glass powder into a $6.4 \mathrm{~mm}$ diameter cylindrical mold using a hydraulic press at 17.2 $\mathrm{MPa}$. The discs were annealed at the same temperature which they were enameled ( $T_{\text {coat, }}$ Table 2.2) in order to promote their coalescence and sturdiness. Three discs of each glass composition were stacked so that the total sample thickness was $12 \mathrm{~mm}$. The samples were tested between 25 and $300^{\circ} \mathrm{C}$ at a heating rate of $4^{\circ} \mathrm{C} / \mathrm{min}$ using a Netzsch DIL $402 \mathrm{PC}$ dilatometer (Netzsch Instruments, Burlington, MA, USA). This temperature range was selected to be lower than the glass transition temperature to ensure that the glass did not soften or stick to the sample holder. The CTE was calculated based on the measured length and temperature changes using:

$$
\alpha_{m}=\frac{1}{\Delta T} \frac{\Delta l}{l_{0}}
$$

where $\alpha_{m}$ is the mean CTE of the glass, $l_{0}$ is the initial length of the test specimen, and $\Delta T$ and $\Delta l$ are the changes in temperature and length of the specimen, respectively. A total of 3 specimens were tested for each glass composition. 


\subsubsection{Mechanical testing}

The fracture tests were performed using the load jig developed by Fernlund and Spelt 169 for the testing of structural adhesive joints. By changing the lengths of the links used to couple the arms of a double cantilever beam (DCB) specimen to the load frame, the jig can produce the entire range of mode ratios from pure mode I to nearly pure mode II. Contrary to other mode II tests such as the end-loaded split (ELS) ${ }^{170,171}$ or end notched Flexure (ENF) ${ }^{172}$ in which the loads are transferred between the adherends through contact, the load jig applies the loads to each arm independently, significantly reducing the friction effects between the crack faces that can lead to artificially higher measured $G_{I I C}$.

As described in Section 3.2.5.3, a number of practical problems were encountered when fabricating conventional DCB specimens for glass coating/metal substrate systems, and therefore recommended using a modified bilayer DCB specimen. In such a specimen, shown in Figure.5.1, one adherend is coated with glass, and the other adherend is attached to the glass coating using a layer of epoxy ${ }^{134}$. As will be discussed in Section 5.3.5, the small asymmetry introduced by the presence of the epoxy and the propagation of the crack off the mid-plane resulted in a loading configuration that was not pure mode II. Nevertheless, the ratio of the mode I to mode II components of the strain energy release rate $\left(G_{I} / G_{I I}\right)$ was found to be only $\sim 0.045$. Therefore, the critical strain energy release rate measured under these conditions will henceforth in any case be referred to as $G_{I I C}$. 
a)
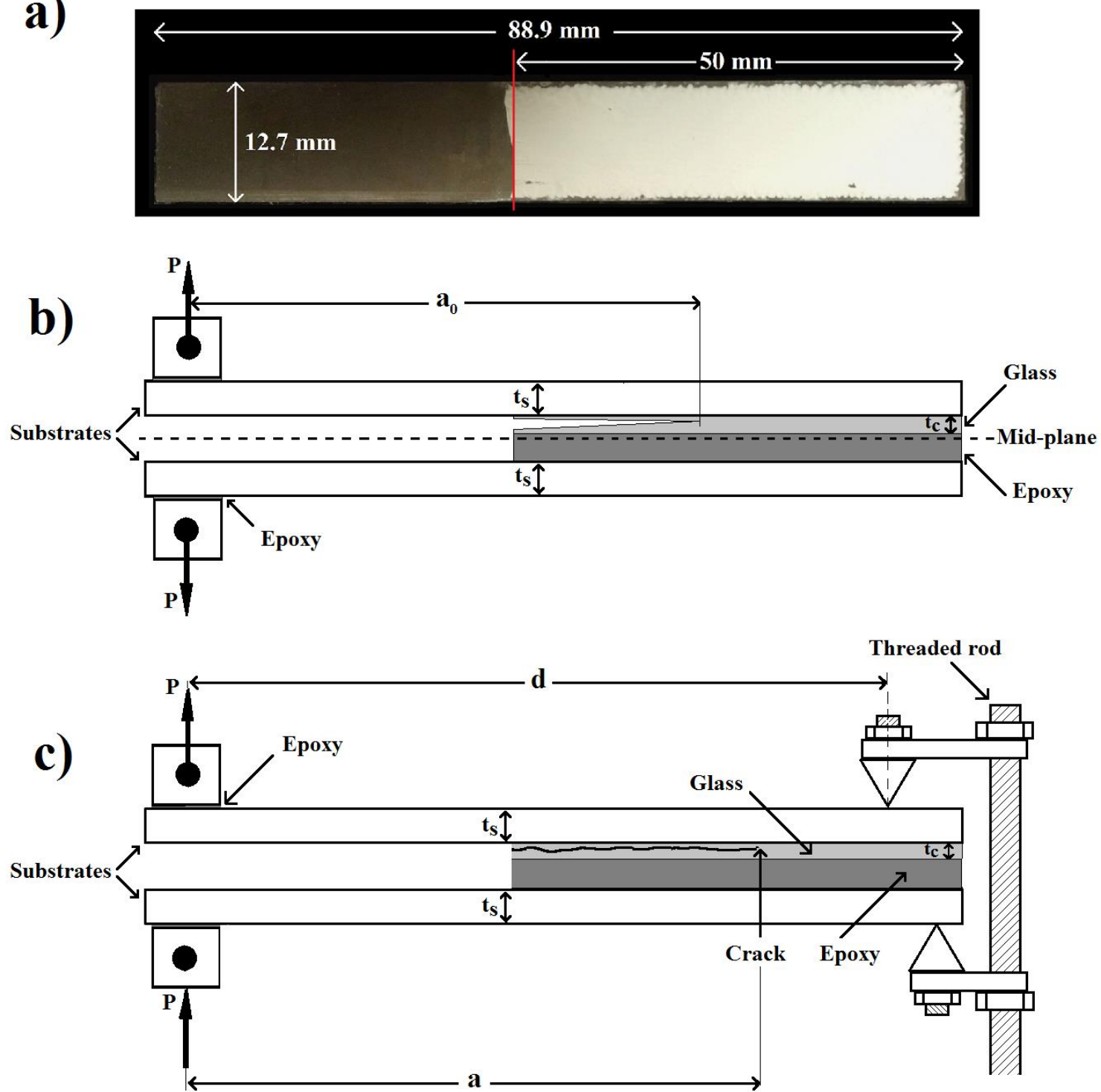

Figure.5.1. (a) Ti6Al4V bar coated with glass, (b) mode I setup for creating the mode I pre-crack (adapted from ${ }^{128}$ ), and (c) mode II setup. Figure is not to scale, i.e. the glass and epoxy layers are shown much thicker than they actually are for clarity.

\subsubsection{Enamelling the bioactive glass onto the metallic}

\section{substrate}

The enamelling procedure followed broadly the same approach as Section 2.3. Briefly, Ti6Al4V plates $(88 \mathrm{~mm} \times 12.7 \mathrm{~mm} \times 3.2 \mathrm{~mm})$ were polished with a fine sand paper (1200 grit) and 
degreased with isopropanol. These dimensions were chosen to satisfy the elastic condition suggested by de Moura and de Morais ${ }^{173}$ which requires $\delta<0.2 d$ where $\delta$ is the adherends' deflection at the points of the applied load and $d$ is the distance from the line of action of the applied forces to the clamp at the root (Figure.5.1). This also ensures that non-linear geometric effects associated with large deflections are negligible.

In order to reduce the residual stresses in the Ti6Al4V plates before they were coated, they were heated unconstrained at a rate of $10^{\circ} \mathrm{C} / \mathrm{min}$ to $650^{\circ} \mathrm{C}$, kept at that temperature for 15 minutes, and then cooled to room temperature at a rate of $0.5^{\circ} \mathrm{C} / \mathrm{min}$. This cooling rate was below the 0.8 ${ }^{\circ} \mathrm{C} /$ min suggested by Donachie ${ }^{133}$ to avoid reintroduction of residual stresses. Under these conditions, Donachie ${ }^{133}$ reported that the residual stresses in Ti6Al4V can be reduced by more than $95 \%$.

An optical non-contact profilometer (Microphotonics Inc, Nanovea, Irvine, CA, USA, ST400) was used to measure the roughness of the plates. For this purpose, five $0.8 \mathrm{~mm}$ traces along the center line of the plate with scan step size of $0.1 \mu \mathrm{m}$ were recorded. Then, a Gaussian filter with a cut-off wavelength of $0.25 \mathrm{~mm}$ was applied on the recorded profile, according to ASME B46.1 ${ }^{131}$, and the roughness was determined.

The coating procedure was according to Section 2.3. The coating temperatures used are outlined in Table 2.1. It is noted that exposing the substrates to high temperatures, in general, may alter the mechanical properties of the titanium alloy. However, the $560-615{ }^{\circ} \mathrm{C}$ temperatures that were used both for coating $\left(560-615^{\circ} \mathrm{C}\right.$ (Table 2.1)), and for reduction of the residual stresses (650 ${ }^{\circ} \mathrm{C}$ ), are considerably lower than the $\alpha+\beta$ transition temperature or $\beta$-transus temperature $\left(995^{\circ}\right.$ $\mathrm{C} \pm 20{ }^{\circ} \mathrm{C}$ ) for Ti6Al4V, according to Donachie ${ }^{133}$. Therefore, changes in metallurgical or mechanical properties of the Ti6Al4V substrates were highly unlikely.

The coating thickness was measured using the optical non-contact profilometer. Three parallel traces (step size of $10 \mu \mathrm{m}$ ) were recorded, $4 \mathrm{~mm}$ apart along the longitudinal axis of the adherend before and after coating. A Gaussian filter with a cut-off wavelength of $0.25 \mathrm{~mm}$ (ASME B46.1 $1^{131}$ ) was applied on the profiles to remove the waviness component. By subtracting the resulting profiles, three thickness profiles per specimen were obtained. For each thickness profile, the point by point averages and standard deviations were calculated and averaged and the overall 
average and standard deviation for each specimen was determined. For each glass coating composition, a total of 3 specimens were tested.

\subsubsection{Residual stress measurement}

A CTE mismatch between the substrate and coating caused the formation of thermal residual stresses in the coating. Since the CTE measurements of Section 5.2.2 were by necessity performed at a lower temperature than the processing temperature of the system, they could not directly be used to determine the residual stresses. Instead, as suggested by Yu et al. ${ }^{132}$, the level of residual stresses can be determined by measuring the change in the curvature of the coated specimens before heating and after cooling down. Figure 5.2 summarizes the three steps required in $\mathrm{Yu}$ et al.'s procedure to determine the residual stresses during the cooling phase of the enamelling process, given the induced signed curvature $k$. It is assumed that the system is stress free at the coating temperature, $T_{\text {Coat }}$, i.e. the temperature at which the bonding occurs. In step 1 , both materials are unconstrained and allowed to cool down to the room temperature. Due to the mismatch in CTE between the coating and the substrate, the coating and substrate contract to different lengths. In step 2, the axial forces $P_{c}$ and $P_{s}$ which are equal in magnitude and opposite in direction, are applied to establish the strain continuity at the interface. The subscripts $c$ and $s$ refer to the bioactive glass coating and the Ti6Al4V substrate, respectively. Finally, in step 3, bending moments $M_{c}$ and $M_{s}$ are applied to maintain moment equilibrium. Therefore, the residual stress is made up of two components, i.e.,

$$
\sigma_{i}=\sigma_{P_{i}}+\sigma_{M_{i}}
$$

where, $\sigma_{P}$ and $\sigma_{M}$ are due to the axial force (step 2 in Figure 5.2), and moment (step 3 in Figure 5.2), respectively, and $i=c$ or $s$ refer to the coating and substrate, respectively. These stresses can be calculated using beam theory as

$$
\sigma_{P_{i}}=\frac{P_{i}}{w_{i} t_{i}}, \sigma_{M_{c}}=-\frac{M_{c}}{I_{c}}\left(z-\frac{t_{c}}{2}\right), \sigma_{M_{s}}=-\frac{M_{s}}{I_{s}}\left(z+\frac{t_{s}}{2}\right)
$$

where

$$
P_{c}=-P_{s}=2 \frac{M_{c}+M_{s}}{t_{s}+t_{c}} ; \quad M_{i}=k E_{i} I_{i}
$$


$z$ is the distance of the layer of interest from the glass coating/ Ti6Al4V substrate interface (Figure 5.4), and $E, M, I, w$ and $t$ are the Young's modulus, bending moment, second moment of area of the cross section, specimen width and thickness, respectively. The width of the glass coating was $12.7 \mathrm{~mm}$, and its thickness is given in Table 5.1. The width and thickness of the Ti alloy substrate were 12.7 and $3.2 \mathrm{~mm}$, respectively. The Young's modulus of the Ti6Al4V substrate was taken as $110 \mathrm{GPa}^{133}$ and the glass coating was assumed to have a Young's modulus of $35 \mathrm{GPa}^{135,136}$, which is similar to that of $45 \mathrm{~S} 5$ bioactive glass.

To obtain the signed curvature $k$, three parallel profiles $4 \mathrm{~mm}$ apart were measured using the optical profilometer with a step size of $10 \mu \mathrm{m}$ along the longitudinal axis on the uncoated side of the substrates, before and after coating. A Gaussian filter with a cut-off wavelength of $0.25 \mathrm{~mm}$ (ASME B46.1 ${ }^{131}$ ) was applied on the profiles to extract the waviness component. Then, by subtracting the profiles before and after coating, the deformed profile was found. Following Yu et $a l .{ }^{132}$, the signed curvature, $k$, of each sample could be found from the maximum deflection of the Ti6Al4V plate $\delta_{R}$, and the half-chordal length $L$ (Figure 5.2), as:

$$
|k|=\frac{2 \delta_{R}}{\delta_{R}{ }^{2}+L^{2}}
$$

$k$ is assumed positive if the unit tangent rotates counter-clockwise, and negative if it rotates clockwise ${ }^{174}$. Even though the measured $\delta_{R}$ values were only on the order of $10 \mu \mathrm{m}$, the profilometer allowed measuring deflections as small as $40 \mathrm{~nm}$, resulting in a relatively low associated error $(<1 \%)$ in the calculated $\sigma_{i}$. 


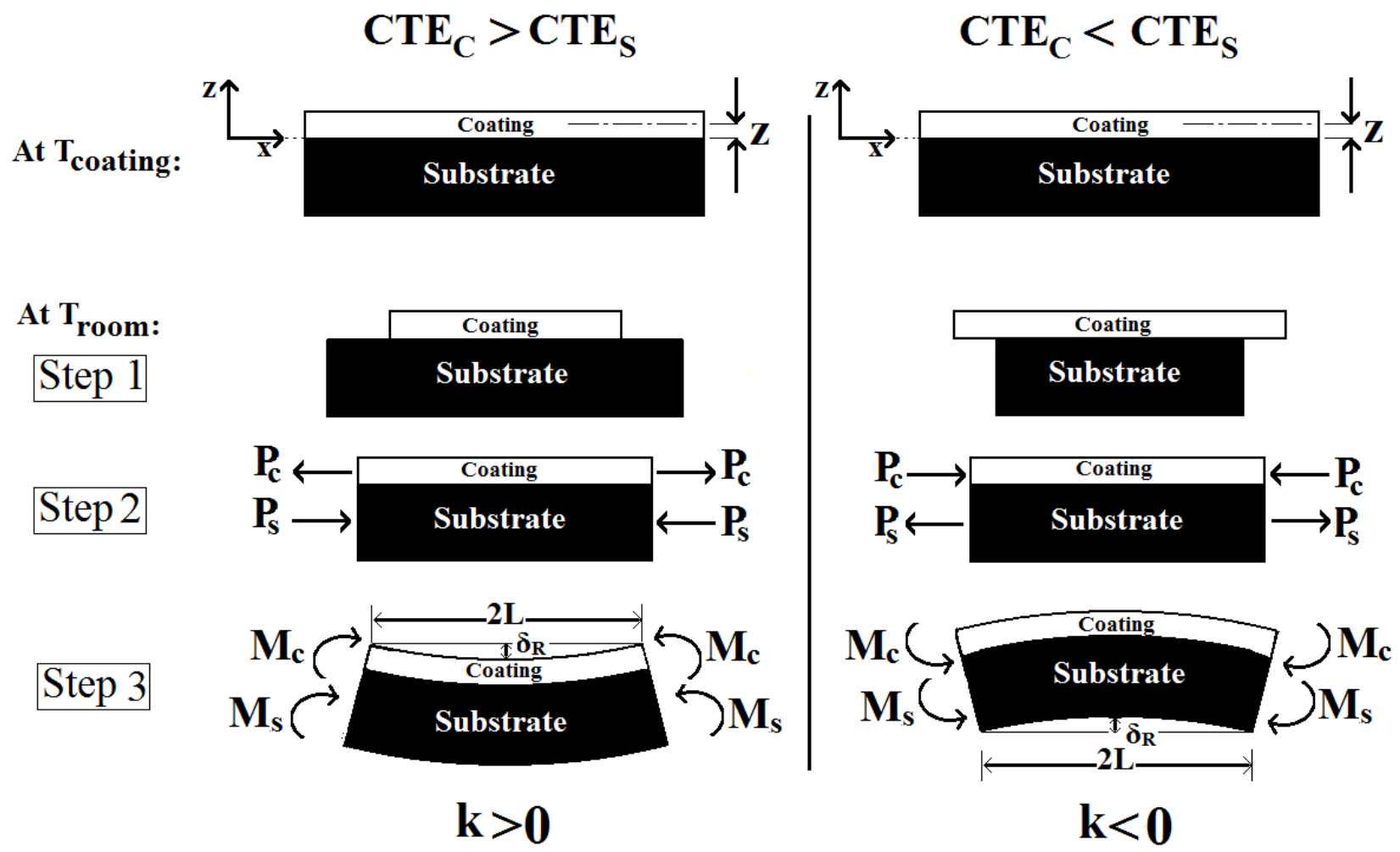

Figure 5.2. Three steps in the analysis of residual stresses during cooling phase of the coating process based on $\mathrm{Yu}$ et al. ${ }^{132}$. $T_{\text {Coat }}$ refers to the temperature at which the bonding occurs, and $T_{\text {room }}$ is room temperature ${ }^{132}$.

\subsubsection{Bilayer double cantilever beam (DCB) specimens}

As mentioned previously and described in detail in Matinmanesh et al. ${ }^{128}$, conventional DCB specimens could not be created with a uniform layer thickness because the alcohol in the mixture used to apply the coating to the substrate evaporated. Therefore, similar to Matinmanesh et al. ${ }^{128}$, bilayer sandwich specimens were made instead (Figure.5.1). Briefly, a room temperature cure epoxy (J-B Weld 8265-S Cold Weld Compound, Sulphur Springs, TX, USA) was applied on each of the glass-coated adherends using a small spatula, and used to attach the coated to the uncoated adherends. The thickness of the epoxy layer was controlled by inserting a $0.2 \mathrm{~mm}$ diameter wire around the edge of the coated area. The wire was removed once the epoxy was set. Then, the DCB specimen was left at room temperature for 48 hours to allow complete curing of the epoxy. 


\subsubsection{Determination of $G_{I I C}$}

Creating a mode I pre-crack prior to performing a mode II test is essential as it creates a sharper crack and a more easily detectable start condition ${ }^{175-178}$ than one made in mode II. Moreover, it has been shown that the measured $G_{I I C}$ values of specimens without pre-cracks are artificially higher than their pre-cracked counterparts ${ }^{176}$. It is noted that use of the mode I starter crack within the glass layer does not preclude failure at the glass/Ti6Al4V interface. Since the mode I pre-crack shown in Figure.5.1b is not on the midline, and because of the model II loading (Figure.5.1c), the crack will tend to be driven towards the glass/Ti6Al4V interface, i.e., the path that minimizes the mode II stresses ${ }^{141,179}$. If the glass/metal interface is less tough than the glass, the specimen will fail at the interface, whereas if the reverse is true, it will likely propagate close to, but not within the interface ${ }^{179}$. Crack path will be further discussed in Section 5.3.5 .

In order to create the mode I pre-crack, the specimens were loaded in displacement control using a United Universal Tester (STM series, United Testing Systems, Inc., Huntington Beach, CA, USA) with a $500 \mathrm{~N}$ load cell, as shown in Figure.5.1b. The crosshead displacement rate of $0.5 \mathrm{~mm} / \mathrm{min}$ was chosen based on the calculation of the separation rates suggested in the ASTM3433-99 standard ${ }^{130}$. The crack was monitored during the test using a movable digital microscope camera (OptixCam Summit SK2-14X, Roanoke, VA USA) with a high magnification lens (field of view of $3 \mathrm{~mm}$ ). The load was applied to the specimen until the crack propagated and then the pre-crack length, $a_{0}$, was recorded after which the specimen was unloaded and removed. The pre crack length, $a_{0}$, was chosen to meet the stable fracture criteria for a pure mode II test $\frac{a_{0}}{d}>$ 0.57 determined by Fernlund and Spelt ${ }^{169}$.

After creating the mode I pre-crack in the specimens, they were placed in the load jig which allowed the identical loads in the same direction required for the mode II test to be applied using a single load frame actuator. Details of the load jig can be found in Ref. ${ }^{169}$. The clamps shown at the end of the specimen in Figure.5.1c provided resistance to rotation. The precracked specimens were loaded in displacement control at a crosshead displacement rate of $1.5 \mathrm{~mm} / \mathrm{min}$ while the crack was observed with the camera. The constant displacement rate was applied to the specimen until the crack started to propagate. Assuming that a load of $P_{\max }$ is required to propagate the crack located at distance $a$ from the line of action of the loads (Figure.5.1), the mode II critical strain energy release rate can be calculated as ${ }^{169}$ : 


$$
G_{I I C}=\frac{\left(P_{\max } a\right)^{2}}{E_{s} w^{2} t_{s}^{3}}
$$

A total of 3 specimens were tested for each glass composition.

\subsection{Results and discussion}

\subsubsection{Coefficient of thermal expansion (CTE)}

As Figure 5.3 illustrates, the CTE of glasses slightly decreased with increasing amount of incorporated $\mathrm{SrCO}_{3}$. The CTE's of Ly-B0 and Ly-B3 were higher, and the CTE of Ly-B5, was lower, than that of Ti6Al4V.

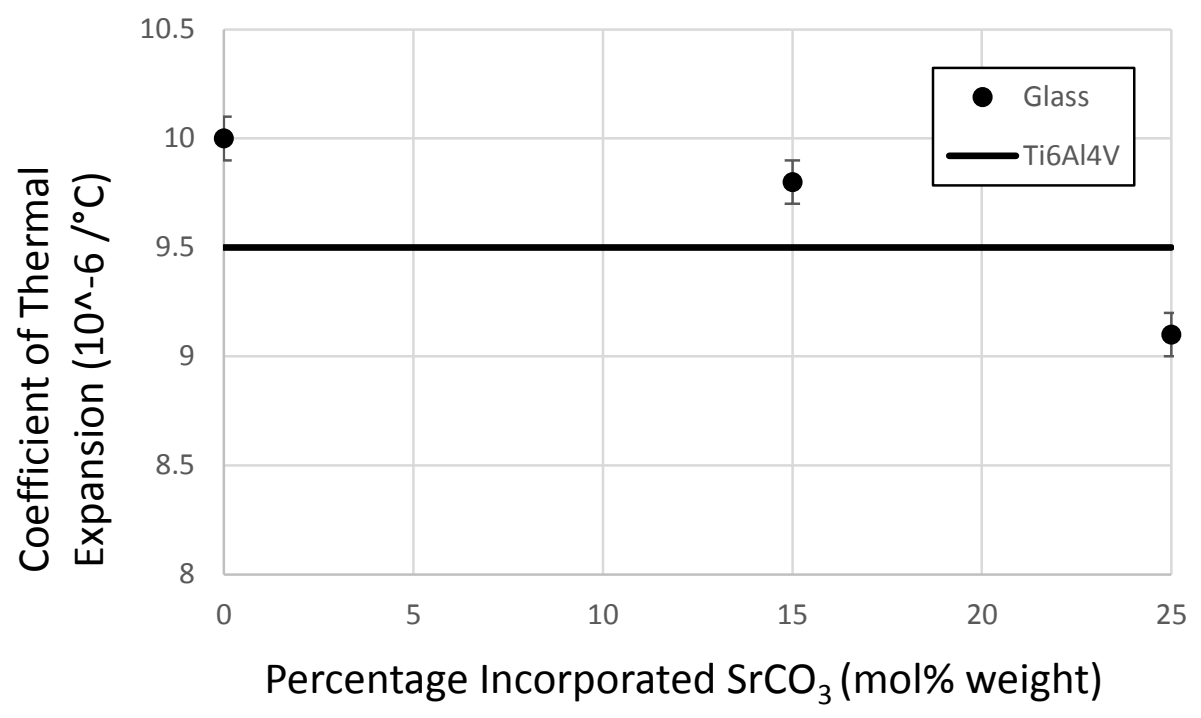

Figure 5.3. The coefficient of thermal expansion (CTE) of the three glass compositions, with the CTE of Ti6Al4V as reference. For each glass composition, 3 specimens were tested. The error bars represent the corresponding standard deviation. The CTE of the Ti6Al4V substrate was taken as $9.5 \times 10^{-6}$ based on the values in the literature ${ }^{154}$. 


\subsubsection{Substrate roughness and coating thickness}

Since the purpose was to investigate the effect of composition and loading configuration on the strain energy release rate of the coating/substrate system, it was essential to ensure that the substrate roughness was relatively constant for all of the plates. After averaging the five measured roughness traces (Section 5.2.3.1), the mean and standard deviation of $R_{a}$ were found to be in the range $0.6-0.7 \mu \mathrm{m}$, and $0.03 \mu \mathrm{m}-0.06 \mu \mathrm{m}$, respectively, for all of the plates. The average coating thickness is shown in Table 5.1.

Table 5.1. Coating thickness $t_{c}$, signed curvature $k$, residual stresses $\sigma_{c}$ and residual coating layer thickness $t_{r}$ for the mode II specimens. The residual stresses are calculated from the deformed substrate profiles as explained in Section 5.2.3.2 .

\begin{tabular}{|c|c|c|c|c|c|}
\hline Glass & Sample & $t_{c}(\mu \mathrm{m})$ & $\begin{array}{c}k(1 / \mathrm{m}) \\
10^{-3}\end{array}$ & $\sigma_{c}(\mathrm{MPa})$ & $t_{r}(\mu \mathrm{m})$ \\
\hline \multirow{4}{*}{ Ly-B0 } & 1 & 98.0 & $4.6 \pm 0.7$ & $10.2 \pm 0.8$ & $16 \pm 4$ \\
\cline { 2 - 6 } & 2 & 103.2 & $5.1 \pm 0.8$ & $10.7 \pm 0.9$ & $17 \pm 5$ \\
\cline { 2 - 6 } & 3 & 91.3 & $3.6 \pm 0.4$ & $8.4 \pm 0.4$ & $14 \pm 4$ \\
\hline \multirow{4}{*}{ Ly-B3 } & 4 & 105.1 & $3.6 \pm 0.2$ & $7.3 \pm 0.3$ & $22 \pm 4$ \\
\cline { 2 - 6 } & 5 & 101.6 & $4.0 \pm 0.2$ & $8.6 \pm 0.3$ & $25 \pm 5$ \\
\cline { 2 - 6 } & 6 & 94.9 & $3.7 \pm 0.3$ & $8.6 \pm 0.3$ & $20 \pm 4$ \\
\hline \multirow{3}{*}{ Ly-B5 } & 7 & 94.0 & $-4.2 \pm 1.0$ & $-9.8 \pm 1.1$ & $19 \pm 3$ \\
\cline { 2 - 6 } & 8 & 87.7 & $-4.0 \pm 1.5$ & $-9.8 \pm 1.4$ & $17 \pm 3$ \\
\cline { 2 - 6 } & 9 & 98.3 & $-3.4 \pm 1.0$ & $-7.6 \pm 1.1$ & $20 \pm 4$ \\
\hline
\end{tabular}




\subsubsection{Residual stresses}

Figure 5.4 presents the total residual stresses calculated from the deformed substrate profiles as explained in Section 5.2.3.2, for one sample from each glass composition. The mean and standard deviations of residual stresses for three traces on each specimen are tabulated in Table 5.1. The glass experienced much larger residual stresses compared to the substrate. The residual stresses in the glass coatings for all three cases did not vary throughout the thickness because they were thin, and the bending component, $\sigma_{M_{c}}$, i.e. the component which changes due to the presence of residual stresses, was significantly lower than the axial one, $\sigma_{P_{c}}$. In the substrate however, the residual stresses were found to strongly depend on the distance from the interface. The total residual stresses in the Ly-B5 glasses measured using the deformed substrate profiles were compressive, indicating their CTE was lower than that of Ti6Al4V, while those in Ly-B0 and Ly-B3 were tensile indicating that their CTE's were larger than Ti6Al4V. Although the CTE measurements given in Figure 5.3 were taken by necessity at a much lower temperature than the processing temperature, the trend in Figure 3 is nevertheless consistent with these results, obtained using the deformed substrate profiles.

a) Sample 1-Substrate

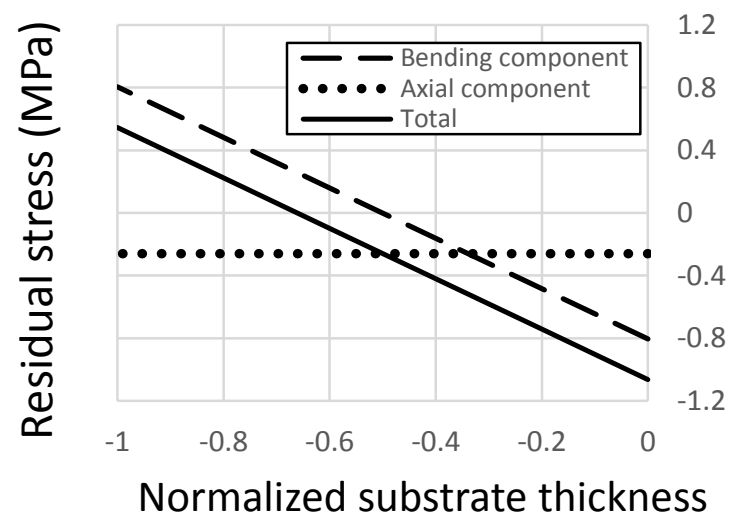

b) Sample 1-Glass

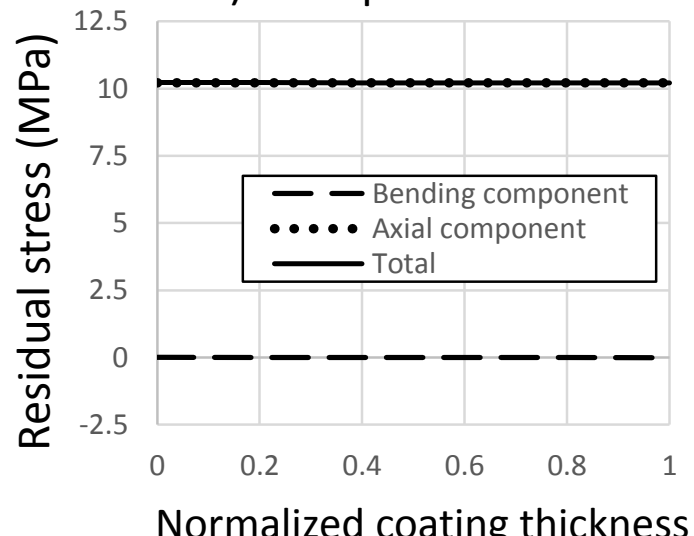

Normalized coating thickness 
c) Sample 4-Substrate

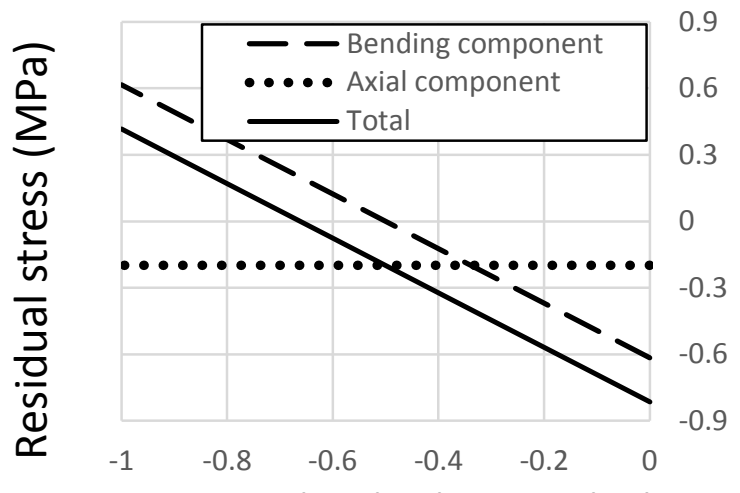

Normalized substrate thickness d) Sample 4-Glass

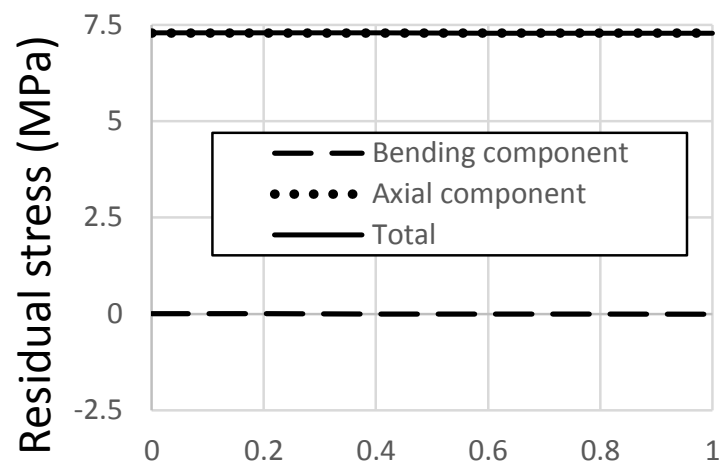

Normalized coating thickness e) Sample 7-Substrate

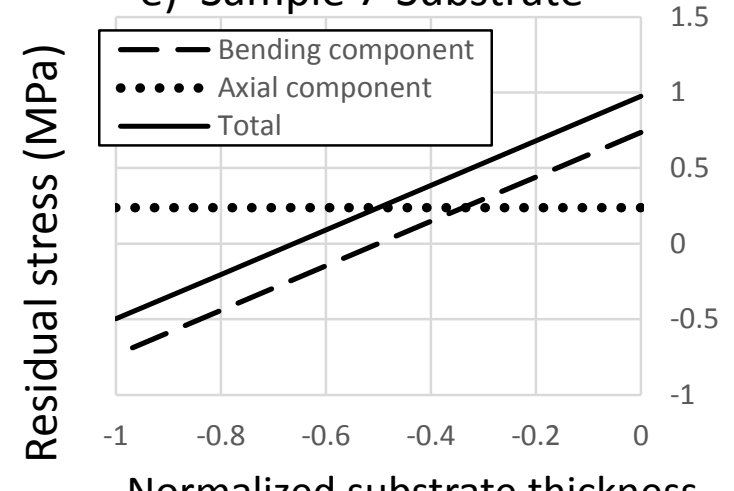

f) Sample 7- Glass

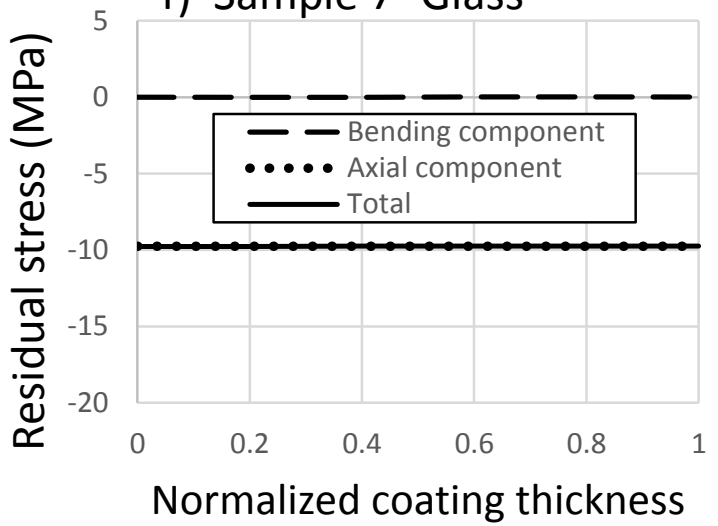

Figure 5.4. Distribution of the residual stresses in the substrate and coating for representative samples of : (a, b) Ly-B0 coating system; (c, d) Ly-B3 coating system; and (e, f) Ly-B5 coating system. The normalized thickness is the distance from the coating/ Ti6Al4V substrate interface, $z$ (Figure.5.1), divided by the total thickness. The residual stresses are calculated from the deformed substrate profiles as explained in Section 5.2.3.2 .

According to Hutchinson and Suo ${ }^{141}$, residual stresses can cause an interfacial crack driving force which is inherently mixed mode, i.e. with a tendency to both open and shear the crack faces. They suggested that the relevant amount of mode I (opening) versus mode II (shearing) to which a planar crack is subjected, depends on the ratio of the thickness of the coating to that of the substrate, $t_{c} / t_{s}$ and the Dundurs parameter, $\alpha$,

$$
\alpha=\frac{\mu_{s}\left(\kappa_{c}+1\right)-\mu_{c}\left(\kappa_{s}+1\right)}{\mu_{s}\left(\kappa_{c}+1\right)+\mu_{c}\left(\kappa_{S}+1\right)}
$$


where the shear modulus, $\kappa_{i}=\left(3-v_{i}\right) /\left(1+v_{i}\right)$ for the plane stress condition, and $\mu_{i}=$ $E_{i} /\left[2\left(1+v_{i}\right)\right]$ and the subscripts $s$ and $c$ represent the substrate and coating, respectively. The Poisson's ratio of the Ti6A14V substrate was taken as $0.34{ }^{133}$. The glass coating was assumed to have Poisson's ratio of $0.26^{137}$, based on values from the literature for $45 \mathrm{~S} 5$ bioactive glass. In the current work, $\alpha \sim 0.53$ and $t_{c} / t_{s} \sim 0.031$ and therefore, based on the relationships presented by Hutchinson and Suo ${ }^{141}$, the 'phase angle' describing the mixed mode nature of the crack driving force caused by the residual stresses,$\psi_{R}=\arctan \sqrt{\frac{G_{I I}}{G_{I}}} \sim 58^{\circ}$ for all the samples. This implies that the interfacial crack driving force caused by the residual stresses tends to shear more than open the crack.

Hutchinson and Suo ${ }^{141}$ suggest that the interfacial crack driving force due to residual stresses, $G_{R}$ can be found using

$$
G_{R}=Z \frac{\sigma_{R}^{2} t_{c}}{E_{c}}
$$

where $\sigma_{R}$ is the residual stress and $Z$ is a dimensionless number which depends on $t_{c} / t_{s}$ and the Dundurs parameter, $\alpha$. Based on the relationships presented by Hutchinson and Suo ${ }^{141}, Z \sim 0.37$ for all samples used in the present study. Using the $\sigma_{R}$ and $t_{c}$ values presented in Table 5.1, the interfacial cracking driving force due to residual stresses is $G_{R}<0.2 \mathrm{~J} / \mathrm{m}^{2}$ in all cases, suggesting that the CTE's of the glasses are relatively close to that of Ti6Al4V, consistent with the CTE results in Figure 5.3. Based on Equation (3.6), the sign of residual stresses (compressive or tensile) does not affect interfacial cracking, i.e., the relevant factor that influences the adhesion is the magnitude of residual stresses rather than their sign. In summary, because the CTEs of the coatings were relatively close to that of the substrate, the residual stresses in the present system did not have a large propensity to cause interfacial fracture.

\subsubsection{Mode II critical strain energy release rate}

In contrast to previous mode I tests which allowed for multiple $G_{I C}$ measurements on a single specimen ${ }^{128}$, no more than a single crack extension event could be recorded with a single mode II test. This is because after creating the mode I pre-crack, little room was left for the mode II crack to propagate. Figure 5.5 presents the mode II critical strain energy release rates $\left(G_{I I C}\right)$ of 
three different glass compositions along with the mode I values from Li et al. ${ }^{167}$. Although the standard deviations brought about by specimen-to-specimen geometric and material deviations are slightly larger for the mode II tests, Figure 5.5 demonstrates that the testing methodology can provide fairly repeatable and reliable measures of toughness.

It is well-known in the literature that materials exhibit a higher resistance to crack propagation in mode II, compared to mode $\mathrm{I}^{180,181}$. This is reflected in Figure 5.5, where for all three glass compositions, the $G_{I I C}$ values are about 3 to 4 times their $G_{I C}$ counterparts. This result is also in reasonable agreement with the findings of Gillanders et al. ${ }^{182}$ who reported $G_{I I C} / G_{I C} \approx$ 4.3 for bulk soda lime glass.

Figure 5.5 demonstrates that the $G_{I I C}$ values increased with increasing $\mathrm{SrCO}_{3}$ content, similar to the trend that was previously reported by Li et al. ${ }^{167}$ for $G_{I C}$. Previous studies ${ }^{183,184}$ indicated that $T_{g}$ positively correlates with Young's modulus, which, in turn, positively correlates with glass fracture toughness ${ }^{185}$. Based on these works, Li et al., ${ }^{167}$, suggested that the increase in the glass transition temperature, $T_{g}$, caused by the addition of $\mathrm{SrCO}_{3}$, could be linked to the increase in $G_{I C}$ of the glasses. Since, as will be discussed in detail in Section 5.3.5, the crack paths were all cohesive, i.e. near the interface, but nonetheless fully within the glass, it is possible that a similar mechanism was also responsible for the mode II trends in Figure 5.5.

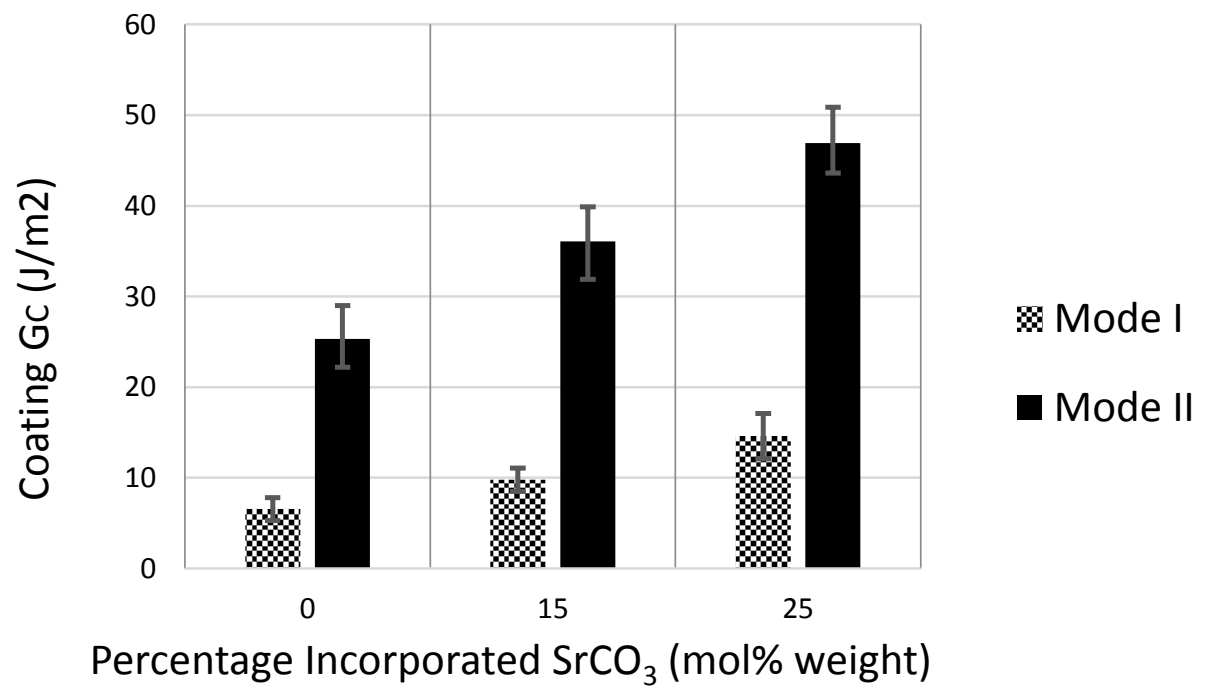


Figure 5.5. Critical strain energy release rate under mode I and mode II loading for three glass compositions. The mode $I$ data is from $\mathrm{Li}$ et al. ${ }^{167}$ and the error bars represent the standard deviation for mode I tests and, min and max for mode II test results.

During mechanical testing, the residual stresses also contribute to the release of the stored strain energy and consequently, less energy from the external loads will be needed to reach the critical energy for crack propagation. Therefore, measurements such as Section 5.2.3.4 lead to an apparent or 'global' $G_{c}$ of the system, rather than the intrinsic fracture toughness. However, in the present case, the ratio of the crack driving force caused by residual stresses to the one caused by the mechanical loading is $\frac{G_{R}}{G_{c}}<5 \%$ in all cases, indicating that the measured $G_{c}$ is very close to the intrinsic fracture toughness of the system.

\subsubsection{Crack path and mode ratio}

In general, the crack in layered materials can grow either 'cohesively' within the layer or 'adhesively' along the interface between the layers. As mentioned in Section 5.2.3.4, the crack was expected to rapidly deflect towards the glass/substrate interface. For this reason, in general, mode II testing is more likely to cause interfacial cracking ${ }^{186}$; however as can be seen in Figure 6, the mode II crack paths were in all cases near-interfacial, i.e., close to the glass/substrate interface, but nevertheless within the glass. This indicates that the mode II fracture toughness of the bulk glass was lower than that of the interface. 


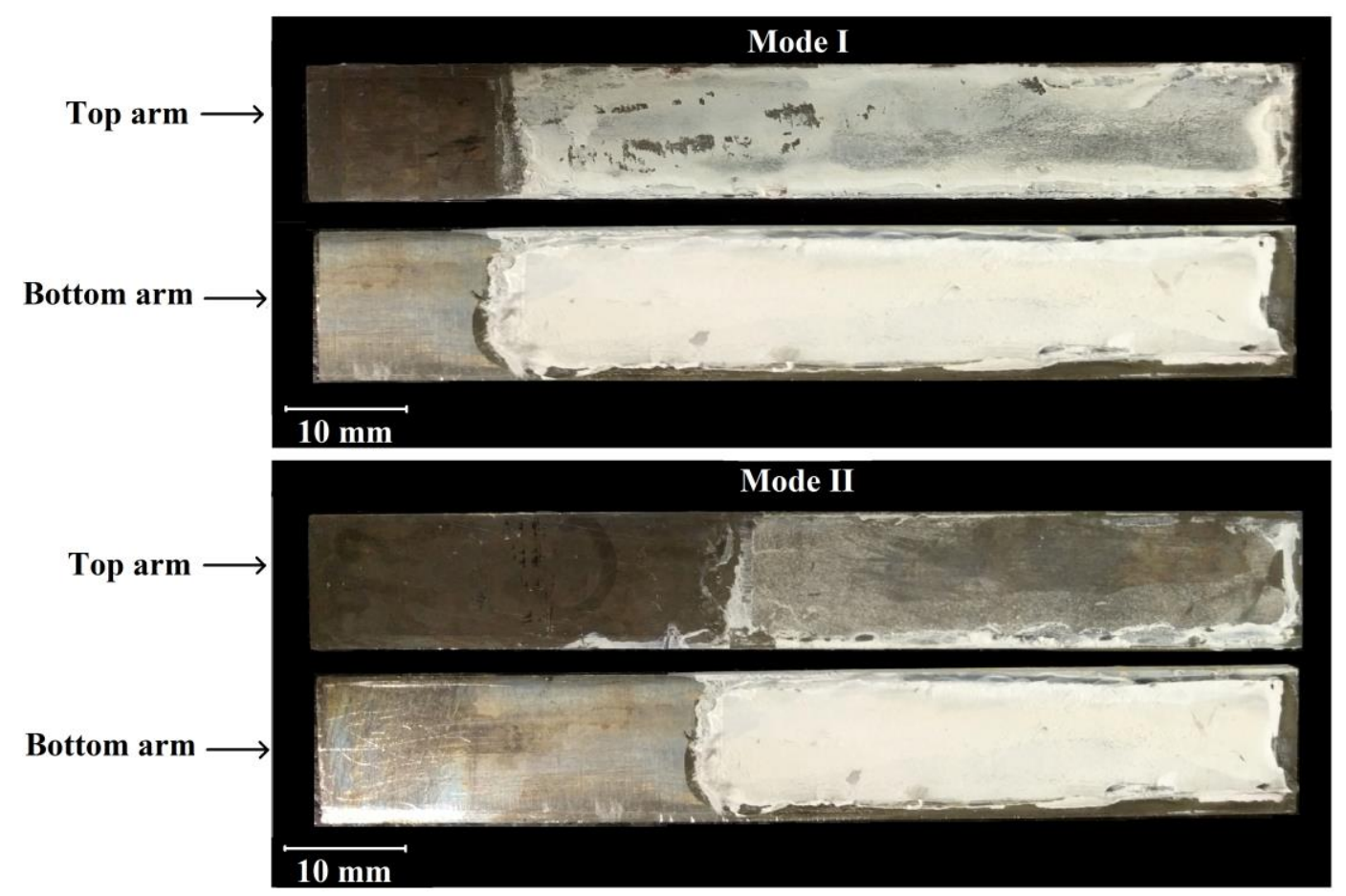

Figure 5.6. Image of two fractured specimens. Bottom arm is the adherend with the epoxy layer under the thicker layer of residual glass.

The values of the residual stresses and coating thicknesses in Table 5.1 were used to calculate the 'T-stress', i.e. the tensile stress within the coating parallel to the crack plane. This parameter can shed light on the effect of the residual stresses on the directional stability of the crack within the coating. The 'T-stress' can be found using ${ }^{144}$,

$$
T \approx \sigma_{R}+\left[\frac{a+1}{\alpha-1}\right]^{0.5} C_{1} K_{I c} t_{c}^{-0.5}
$$

where $\sigma_{R}$ represent the residual stress in the coating, $\alpha$ is one of the Dundurs parameters introduced in Equation (3.8) and $C_{1}$ is a function of the Dundurs parameters that can be found in Ref. ${ }^{141}$. The mode I stress intensity factor, $K_{I C}$, can be determined using the mode I critical strain energy release rate $G_{I C}$ according to the well-known relationship $K_{I C}=\sqrt{G_{I C} E_{C}}$ 82. Equation (3.9) was used for the 9 specimens in Table 5.1 and the T-stresses for all samples were found to be negative, indicating that the crack path was stable, the trajectory straight, and the deviation towards the interface occurred at a small angle in all cases. This is confirmed in Table 5.1, where the standard deviation of the residual layer thickness is less than $5 \mu \mathrm{m}$ in all cases. 
Measurements of residual coating thickness $t_{r}$, on the top arms of the fractured specimens i.e. the substrate without epoxy layer, were used to more closely study the crack path (Table 5.1). Figure 5.7 compares the thicknesses of the residual layers of mode II specimens with their mode I counterparts. No consistent correlation was found between the percentage of the incorporated $\mathrm{SrCO}_{3}$ and the residual layer thickness. However, comparing the results of mode I and mode II, the crack path was closer to the interface between the glass and substrate in the mode II samples than in the mode I counterparts which is expected, and consistent with previous findings in the literature ${ }^{187}$.

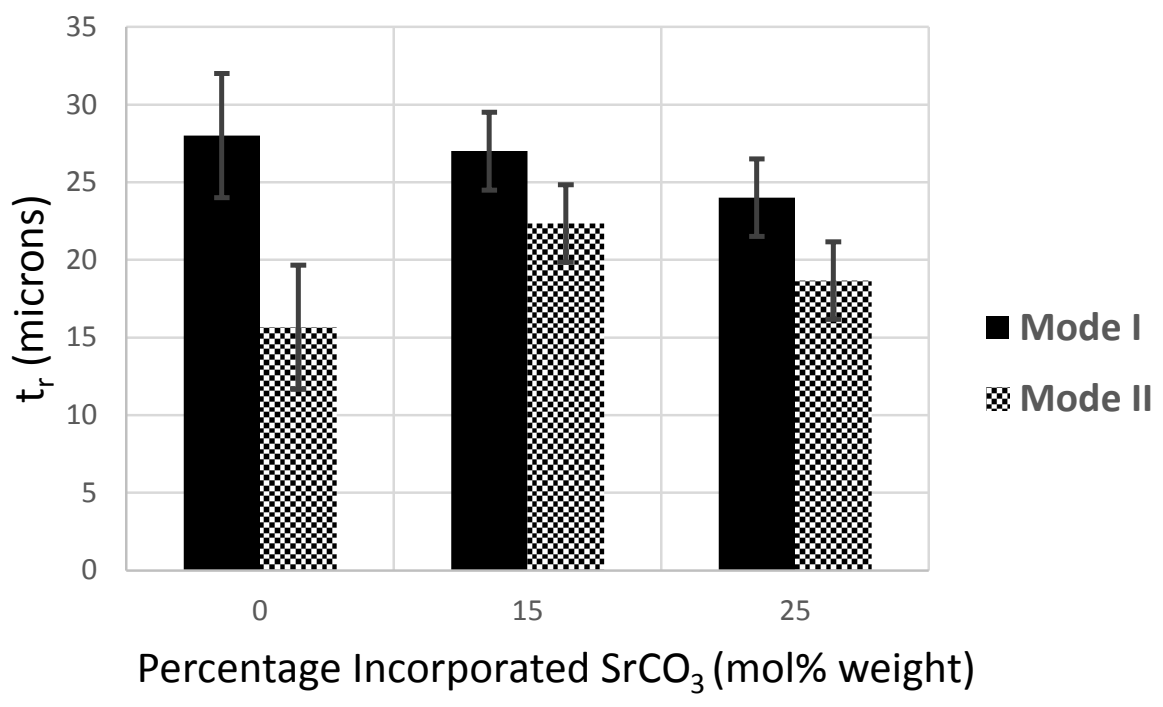

Figure 5.7. Residual layer thickness for mode I and mode II specimens. A lower value indicates a crack that is closer to the Ti alloy/coating interface. The error bars represent the standard deviation for both mode I and mode II test results.

Since the crack paths described above were near the glass/substrate interface rather than in the centre plane of the specimen, and because of the slight asymmetry introduced by the presence of the epoxy layer, the present fracture tests deviated slightly from pure mode II. In this case, taking into account the location of the crack planes inferred from the residual coating thicknesses measured in Table 3, Hutchinson and Suo's general solution ${ }^{141}$ for homogenous isotropic multilayer systems with crack planes located at different locations can be used to calculate the actual phase angle of loading as $\psi_{L}=\arctan \sqrt{\frac{G_{I I}}{G_{I}}}=-78^{\circ}$ for all 9 specimens. This implies $\frac{G_{I}}{G_{I I}} \sim 0.045$ so that the test was very near to pure mode II. It is noted that the negative phase angle 
implies that the loading condition of Figure.5.1c drives the crack extension towards the glass/metal interface $^{179}$.

Finally, it is noted that the present work characterizes the bioactive glass coatings before they degrade when subjected to body fluids. The effect of degradation on the measured mode I and mode II critical strain release rates, crack path and residual stresses is a very important topic for further studies.

\subsection{Chapter summary}

In this chapter, a fracture mechanics based coating/substrate system testing methodology was presented and used to measure the mode II critical strain energy release rate $\left(G_{I I C}\right)$ of a series of borate-based bioactive glass coatings on Ti6Al4V substrates. This methodology was demonstrated to provide repeatable and reliable $G_{I I C}$ measurements. Using this technique, it was found that increasing $\mathrm{SrCO}_{3}$ content in the coatings $(0,15 \mathrm{~mol} \%$ and $25 \mathrm{~mol} \%$ respectively at the expense of $\mathrm{B}_{2} \mathrm{O}_{3}$ ), almost doubled the $G_{I I C}$ from 25.3 to $46.9 \mathrm{~J} / \mathrm{m}^{2}$. The measured $G_{I I C}$ values were about 3 to 4 times their $G_{I C}$ counterparts, measured in an earlier study. The residual stresses in the coating were measured and it was found that the Ly-B5 glass coating incorporating the most $\mathrm{SrCO}_{3}$ experienced compressive stresses, while the Ly-B0 and Ly-B3 which had lower or no $\mathrm{SrCO}_{3}$ experienced tensile residual stresses. The distribution of residual stresses in the coatings were found to distribute uniformly over the cross section of the layer due to its relatively low thickness. Since the CTE's of borate glasses are generally close to that of Ti6Al4V, the crack driving force caused by the residual stresses was found to be negligible compared to that caused by the mechanical loading, indicating that the measured $G_{c}$ was very close to the intrinsic fracture toughness of the system.

As expected, the mode II loading drove the mode I pre-crack towards the glass/substrate interface while the negative T-stress ensured that the deviation occurred at a small angle. The crack remained cohesive for all specimens, indicating that the bulk glass fracture toughness was lower than that of the interface. Overall, the present work in this chapter demonstrated that the developed technique can provide a quantitative measure of the critical strain energy release rate of bioactive glass coating/ metallic substrate system under shear loading. 
The fracture testing methodology developed in this chapter and Chapter 3 will be used in Chapter 6 to investigate the effect of degradation on the fracture of the coating/substrate system. 
6. Evaluating the critical strain energy release rate of bioactive glass coating/Ti6Al4V after degradation 
The work contained in this chapter has been accepted for publication as follows ${ }^{188}$ :

Matinmanesh A., Li, Y., Nouhi A., Zalzal .P, Schemitsch E. H., Towler M. R., Papini M. Evaluating the critical strain energy release rate of bioactive glass coatings on Ti6Al4V substrates after degradation, Journal of mechanical Behaviour of Biomedical materials (Accepted).

\subsection{Introduction}

In Chapter 5 the developed fracture testing methodology was applied to quantify the nearly mode I and mode II critical strain energy release rate of a series of borate bioactive glass coating/Ti6Al4V substrate systems and it was found that the resistance of such systems is 3 to 4 times higher under shearing compared to opening loads. The results of all the previous chapters are focused only on the coatings' mechanical performance before degradation and there appears to be no existing studies which determine the fracture toughness of degraded bioactive glass coating /metal systems. This chapter adapts the fracture mechanics based methodologies presented in Chapters 3 and 5 to investigate the effect of degradation on the resulting mode I and II critical strain energy release rates. The effect of incorporating different amounts of $\mathrm{SrCO}_{3}$ in the glasses on the dissolution rate and critical strain energy release rates, and the correlation between the residual stresses in the coating and their dissolution rate were was also studied.

\subsection{Experiments}

\subsubsection{Specimen preparation}

\subsubsection{Glass preparation}

Three glasses, Ly-B0, Ly-B3 and Ly-B5, were prepared based on the procedure outlined in Section 2.2. 


\subsubsection{Coating of Ti6Al4V substrates}

Ti6Al4V plates (McMaster-Carr, Elmhurst, Illinois, USA) with dimensions $88.9 \mathrm{~mm} \times 12.7 \mathrm{~mm} \times 3.2 \mathrm{~mm}$ and roughness of $0.5-0.6 \mu \mathrm{m}$ were cleaned using ethanol. The residual stresses in the Ti6Al4V plates were released by heating at $650^{\circ} \mathrm{C}$ for 15 minutes with no constraints and letting them slowly cool inside the furnace.

Mode I and mode II coating specimens were prepared according to the procedure outlined in Section 2.3. In order to create a stable crack propagation condition, $13.9 \mathrm{~mm}$ [according to ASTM $3433^{130}$ ] and $38.9 \mathrm{~mm}$ [according to Wang and Vu-Khanh ${ }^{170}$ ] of the glass was scraped off from the end of substrates before heating, leaving $75 \mathrm{~mm} \times 12.7 \mathrm{~mm}$ and $50 \mathrm{~mm} \times 12.7 \mathrm{~mm}$ area glass layers on mode I (Figure 6.1 (a)) and mode II samples (Figure 6.1 (b)), respectively.
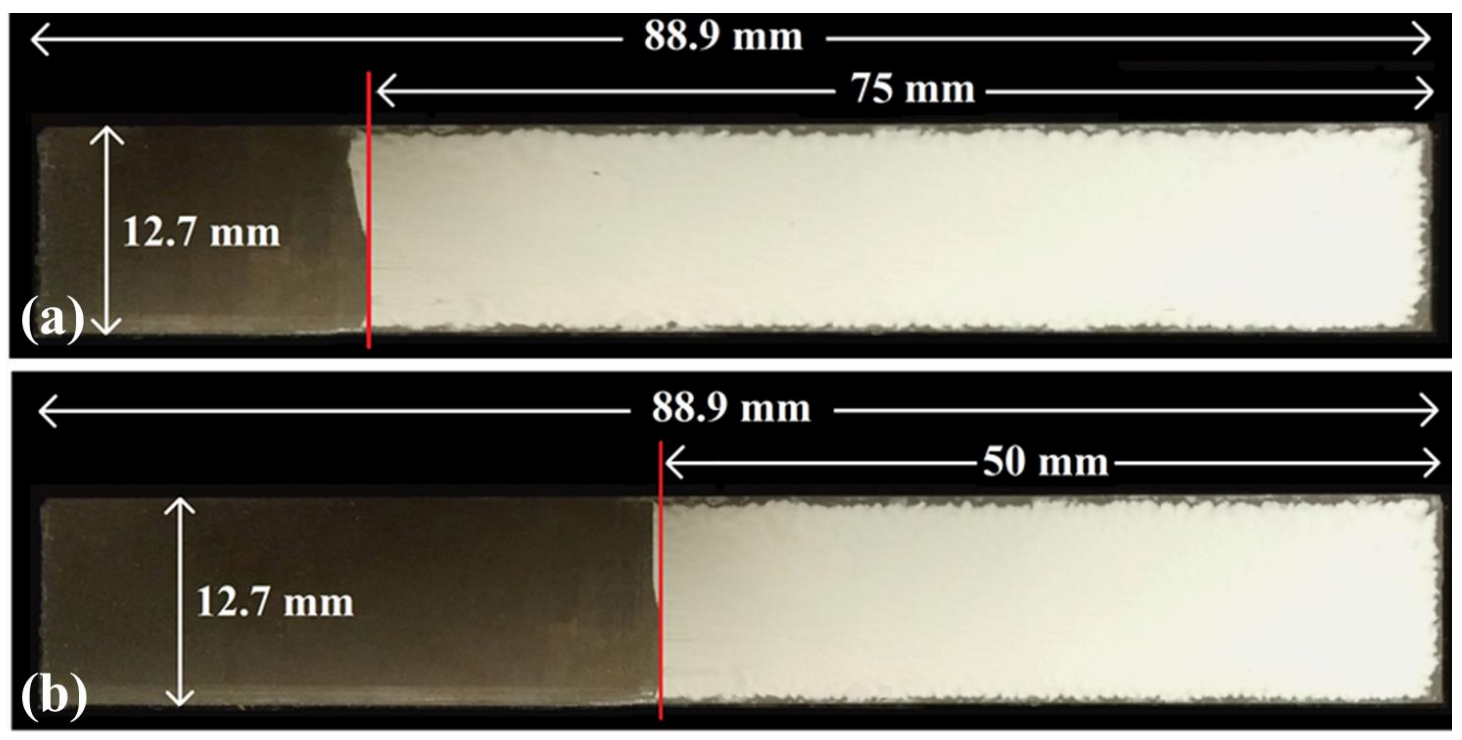

Figure 6.1. Enamelled glass coating open faced specimens before degradation for (a) mode I and (b) mode II tests. In order to create a stable crack propagation condition, $13.9 \mathrm{~mm}$ (according to ASTM $3433^{130}$ ) and $38.9 \mathrm{~mm}$ (according to ${ }^{170}$ ) of the glass was scraped off from the end of substrates. 


\subsubsection{Specimen Characterization}

\subsubsection{XRD analysis}

A D2 PHASER (Bruker AXS Inc., WI, USA) was used to collect the x-ray diffraction patterns. Glass powders and coated Ti6A14V substrates were stuck onto stainless steel sample holders using double-sided adhesive. The diffractograms were collected in the range of $20^{\circ}<2 \theta$ $<80^{\circ}$ at a scan step size $0.02^{\circ}$ and a count time of $0.3 \mathrm{~s}$, where $30 \mathrm{kV}$ generator voltage and 10 $\mathrm{mA}$ tube current were applied.

\subsubsection{Residual stress measurement}

The magnitude and distribution of thermal residual stresses induced in the coating due to the CTE mismatch between the undegraded bioactive glass coatings in Table 2.1 and the Ti6Al4V substrates were previously determined in the previous chapter (Section 5.3.3 ). In order to investigate whether the residual stresses were affected by degradation, as in $\mathrm{Yu}$ et al. ${ }^{132}$, the profile of the substrates were recorded and compared before and after degradation. To do this, the optical profilometer was used to record three parallel profiles along the longitudinal axis, $4 \mathrm{~mm}$ apart (step size of $10 \mu \mathrm{m}$ ) from the uncoated side of the substrates, before and after degradation. After extracting the waviness component of the profiles by the use of Gaussian filter (cut-off wavelength of $0.25 \mathrm{~mm}$ (ASME B46.1 ${ }^{131}$ ), the resulting profiles before and after degradation were subtracted point by point to obtain the deformed profiles. A total of 3 specimens per glass composition were tested for each degradation period.

\subsubsection{Degrading the coatings and measuring the weight loss}

The degradation of the coatings was assessed gravimetrically after 2, 6 and 24 hours of immersion in de-ionized water. The weights of Ti6Al4V substrates immediately before $\left(W_{0}\right)$ and after $\left(W_{l}\right)$ the coating procedure were measured, then the weights of the coated specimens after degradation were measured as $W_{2}$. The percentage weight loss $(\Delta W)$ was calculated using an analytical scale (accuracy $0.0001 \mathrm{~g}$ ) as 


$$
\Delta W=\frac{W_{1}-W_{2}}{W_{1}-W_{0}} \times 100
$$

A total of 3 specimens per glass composition were tested for each degradation period.

\subsubsection{Coating thickness before and after degradation}

The thicknesses of the coatings both before and after degradation were measured. The thickness before degradation was necessary to ensure relative uniformity of specimens, and, after degradation, was used to further characterize the degradation. Three traces, each $4 \mathrm{~mm}$ apart, along the longitudinal axis of the Ti6Al4V plates were recorded (step size of $10 \mu \mathrm{m}$ ) using an optical non-contact profilometer (Microphotonics Inc, Nanovea ST400), both before and after the enamelling. The waviness was then removed by Gaussian filtering the profile (cut-off wavelength of $0.25 \mathrm{~mm}$ (ASME B46.1 ${ }^{131}$ )). The difference in the resulting profiles was calculated, and three coating thickness profiles per specimen were obtained. The mean and standard deviation of each thickness profile were calculated and averaged to determine the overall mean and standard deviation for each specimen before degradation. A total of 3 specimens were tested for each glass composition

In order to measure the change in thickness due to degradation, the three thickness profiles were recorded again from the degraded samples and point-by-point differences of the profiles before and after degradation were calculated to obtain three thickness-change profiles per specimen. The mean and standard deviation of each of these profiles were calculated and averaged to find the change in the coating thickness due to degradation. Ly-B0 (2h, $6 \mathrm{~h}$ and $24 \mathrm{~h}$ ) and Ly-B3 (24h) coatings delaminated after degradation, which made the thickness change measurement impossible. This will be further discussed in Section 6.3.4. A total of 3 specimens per glass composition were tested for each degradation period.

\subsubsection{Measurement of $G_{I C}$ and $G_{I I C}$}

In order to study the effect of degradation on the $G_{I C}$ and $G_{I I C}$ of the degraded glass coating/Ti alloy systems, the procedures described in Section 3.2.5.4 5.2.3.4 and Section 5.2.3.4 were used to create bi-layer DCB specimens (Figure 6.2). For both cases, a layer of epoxy (J-B 
Weld 8265-S Cold Weld Compound, Sulphur Springs, TX, USA) was used to attach each of the degraded coated specimens to an uncoated substrate of the same size. The thickness of the epoxy layer was controlled by inserting a $0.2 \mathrm{~mm}$ diameter wire around the edges of the coated specimen, which was removed after the epoxy set. Quick set epoxy was used to glue the two hinges, for mode I, and the loading blocks, for mode II specimens, to the free ends of the plates (Figure 6.2).

As discussed in Section 3.3.7 and Section 5.3.5, introduction of the epoxy layer and advancement of the crack off the mid-plane causes a small asymmetry in the configurations shown in Figure 6.2. Nevertheless, the mode ratio associated with Figure 6.2a and Figure 6.2b were reported to be $\frac{G_{I I}}{G_{I}}=0.020$ (near pure mode I) ${ }^{152}$ and $\frac{G_{I}}{G_{I I}}=0.045$ (near pure mode II) ${ }^{168}$, respectively. Therefore, the critical strain energy release rate measured by these configurations will from now on be referred to as $G_{I C}$ and $G_{I I C}$. 

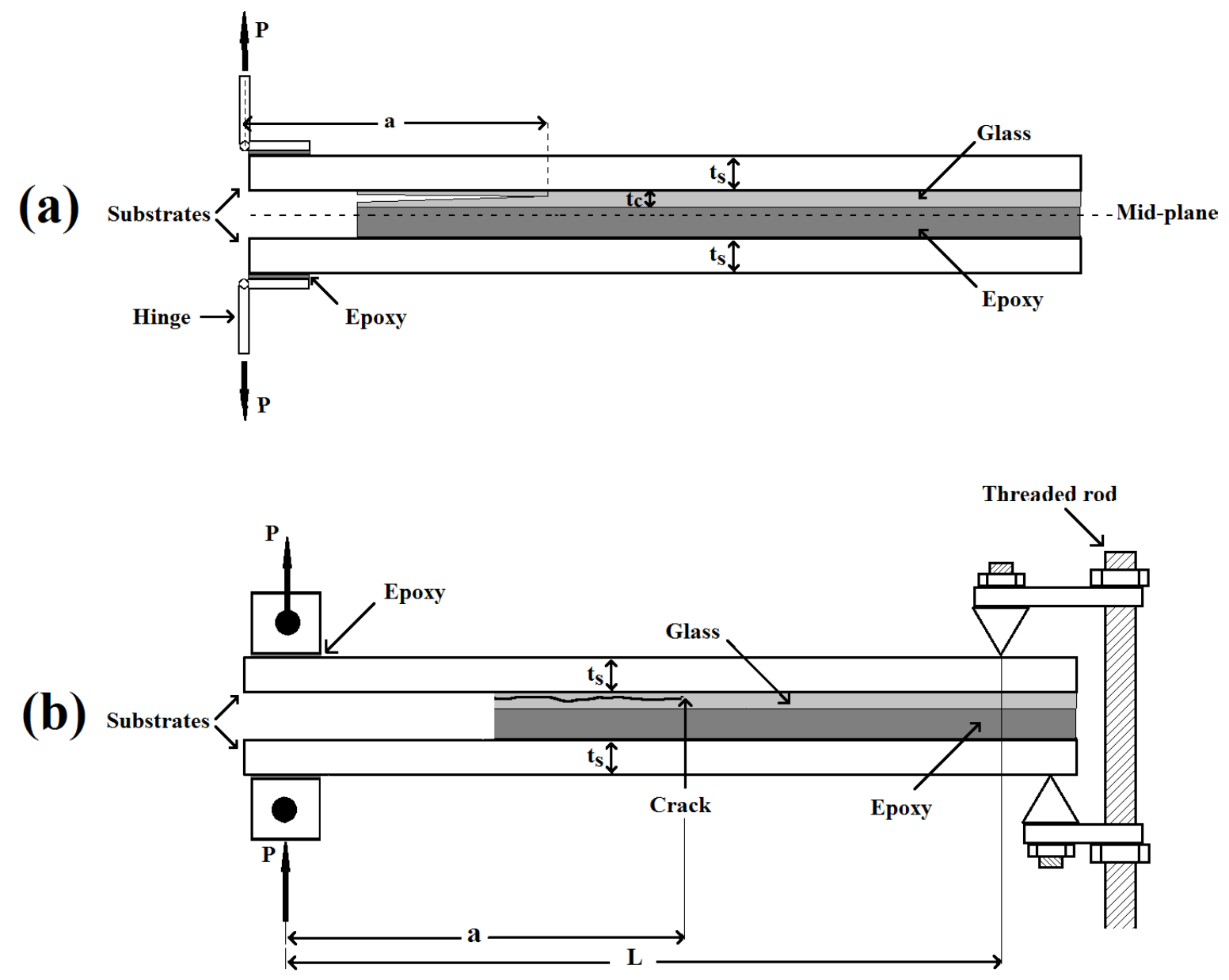

Figure 6.2. Double layer DCB used for mode I (a) ${ }^{128}$ and Mode II (b) ${ }^{168}$ fracture tests.

The mode I tests were based on the procedure presented by 3.2.5.4 . Briefly, identical magnitude but opposite direction loads were applied to the two arms of the mode I specimens (Figure 6.1a) with a displacement rate of $0.5 \mathrm{~mm} / \mathrm{min}$ using a United Universal Tester (STM series, United Testing Systems, Inc., Huntington Beach, CA, USA) equipped with a $500 \mathrm{~N}$ load cell. The propagation of the crack was recorded using a movable digital microscope camera (OptixCam Summit SK2-14X, Roanoke, VA USA) with a field of view of $3 \mathrm{~mm}$. By stopping and restarting the crosshead displacement after every crack propagation, between 2 to 4 measurements of $G_{I C}$ per specimen were possible. Three specimens per glass composition were tested. The $G_{I C}$ was calculated using ${ }^{82}$ : 


$$
G_{I C}=\frac{12 P_{\max }^{2} a^{2}}{E_{s} w^{2} t_{s}^{3}}
$$

where $P_{\max }$ is the force required to propagate the crack, $E_{s}, w$ and $t_{s}$ are the modulus of elasticity, width and thickness of the substrate, respectively and $a$ is the distance from crack tip to the loading point (Figure 6.2). A total of 3 specimens per glass composition were tested for each degradation period.

The mode II fracture tests were based on the procedure described in Section 5.3.4 using the load jig of Fernlund and Spelt ${ }^{169}$. First, the mode II specimens (Figure 6.1b) were loaded in mode I loading condition to create a mode I pre-crack. The mode I pre-crack has been previously shown to create a sharper and therefore more detectable crack ${ }^{175-178}$ and also prevent measurement of artificially high mode II fracture toughness values for the system ${ }^{176}$. The specimens were then loaded in mode II using the load jig using a crosshead displacement rate of $1.5 \mathrm{~mm} / \mathrm{min}$. The jig link lengths were chosen such that two equal magnitude forces were applied to each arm in the same direction. The crack was monitored using the movable camera as described above. After recording the loads at which the crack propagated, the mode II critical strain energy release rate was calculated as ${ }^{169}$ :

$$
G_{I I C}=\frac{9\left(P_{\max } a\right)^{2}}{E_{s} w^{2} t_{s}^{3}}
$$

A total of 3 specimens per glass composition were tested for each degradation period.

\subsubsection{Roughening of substrates}

In order to investigate the effect of substrate roughness on the mode I and mode II critical strain energy release rate of the degraded coating/substrate systems, the Ti6Al4V plates were roughened using an AccuFlo AF10 Micro Abrasive Blaster (Comco, Inc. Burbank, CA, USA), equipped with a dehumidifier, a desiccant filter on air inlet and a refrigeration air dryer to minimize the moisture in the power reservoir. Aluminum Oxide $\left(\mathrm{Al}_{2} \mathrm{O}_{3}\right)$ particles (Comco, Inc. Burbank, CA, USA) with nominal diameters of $150 \mu \mathrm{m}$ (Weibull distribution, mean: 182, standard deviation: 41) were blasted through a nozzle (Comco, Inc. Burbank, CA, USA) with diameter of $1.5 \mathrm{~mm}$ and standoff distance of $25 \mathrm{~mm}$ at $600 \mathrm{kPa}$ air pressure and mass flow rate of $25.3 \mathrm{~g} / \mathrm{min}$. 
The substrates were clamped on a programmable motorized stage (Aerotech Inc., Pittsburgh, PA, USA) that allowed linear motion under the fixed jet in two directions independently with positioning resolution of $5 \mu \mathrm{m}$. The stage was programed to have a transversal oscillation (with frequency of $7 \mathrm{~Hz}$ and displacement amplitude of $5 \mathrm{~mm}$ ) as it moved with a constant velocity along the longitudinal axis of the substrate, in order to ensure a uniform coverage of the entire area of the substrate. The longitudinal velocity was set at $1 \mathrm{~mm} / \mathrm{s}$ and $25 \mathrm{~mm} / \mathrm{s}$ to create two levels of increased roughness on the substrates.

The roughness of all the substrates (as-received and roughened) were measured using an optical non-contact profilometer (Microphotonics Inc, Nanovea ST400) by recording five $0.8 \mathrm{~mm}$ traces along the center line of the substrates with scan step size of $0.1 \mu \mathrm{m}$ as per ASME B46.1 ${ }^{131}$. Then, a Gaussian filter with a cut-off wavelength of $0.25 \mathrm{~mm}$ (ASME B46.1 ${ }^{131}$ ) was applied on the recorded profile and the average roughness, $R_{a}$, for each roughness profile was calculated and averaged to determine the overall $R_{a}$ of the substrate. The as-received and roughened substrates were then coated with the glass coatings according to the procedure of Section 0. The coated specimens were degraded for $2 \mathrm{~h}, 6 \mathrm{~h}, 24 \mathrm{~h}$ and 3 days as per Section 6.2.2.3 and tested under mode I and mode II loading conditions as per Section 6.2.2.5 . A total of 3 specimens per glass composition were tested for each degradation period.

\subsection{Results and Discussion}

\subsubsection{XRD Analysis}

The XRD patterns of the three glasses in their as fired forms and as enamelled coatings on the Ti6Al4V substrates are presented in Figure 6.3 (a) and (b), respectively ${ }^{167}$. The glassy nature of the original glasses and coatings were confirmed by the broad XRD patterns without any detectable peaks. Since the crystalline phases can influence the solubility, biocompatibility and

mechanical properties of the glasses ${ }^{189}$, it is required to keep the amorphous structure of all the coatings. 
(a)

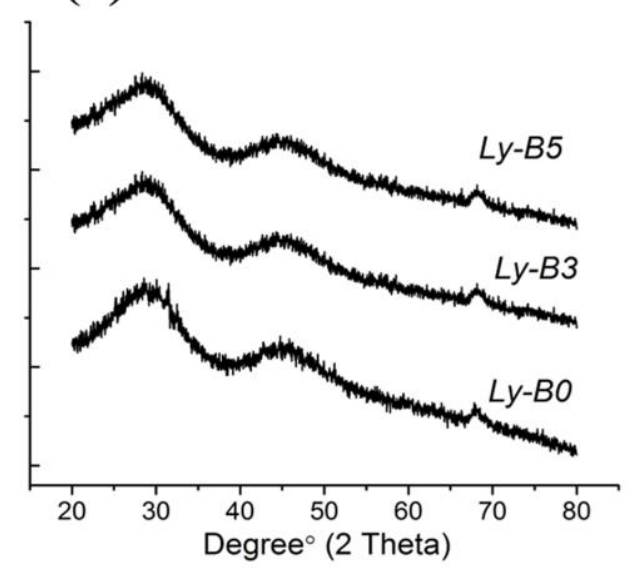

(b)

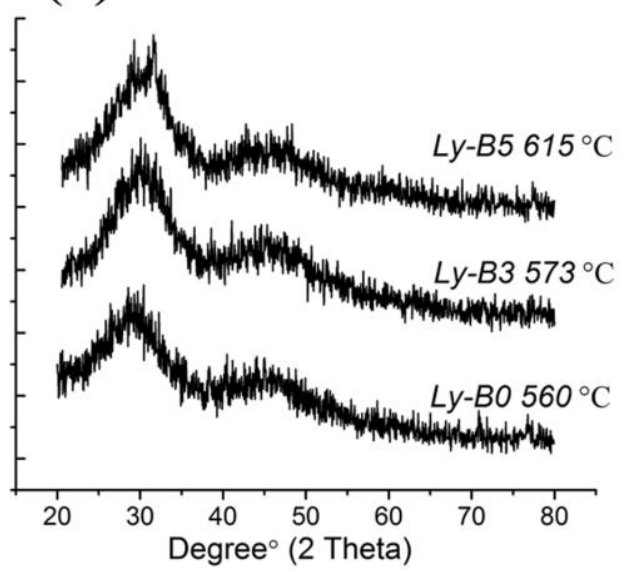

Figure 6.3. The XRD patterns of (a) the glasses and (b) glass coatings on the Ti6Al4V substrates, where the coating processing temperatures were tagged ${ }^{167}$.

\subsubsection{Residual stresses}

It was reported in Table 5.1 that the induced signed curvature $(k)$ in the substrates due to the CTE mismatch between the un-degraded bioactive glass coatings and Ti6Al4V plates to be $4.4 \pm 0.6\left(10^{-3} / \mathrm{m}\right), 3.8 \pm 0.3\left(10^{-3} / \mathrm{m}\right)$ and $-3.9 \pm 1.2\left(10^{-3} / \mathrm{m}\right)$ for Ly-B0, Ly-B3 and Ly-B5, respectively. The change in the substrate's curvature after degradation was determined according to the procedure in Section 6.2.2.2 and was found to be less than $0.2\left(10^{-3} / \mathrm{m}\right)$ in all cases, which falls within the standard deviation range of the data reported by Table 5.1. This suggests that the change in the residual stresses due to degradation was very small compared to the values of residual stresses reported for the un-degraded bioactive glass coatings. ${ }^{168}$

\subsubsection{Weight loss due to degradation}

Since ion release from the glass is accompanied by a decrease in mass, weight loss measurements can be considered as a useful parameter for monitoring the kinetics of glass solubility. The weight losses of the glass coatings at different degradation times are shown in Figure 6.4. The substrates used for the weight loss study (Figure 6.4) were the 'as-received' (not roughened) substrates. For all incubation times, the weight loss of the glass coatings decreased 
with increased addition of $\mathrm{SrCO}_{3}$, indicating that the degradation rate of the glass coatings decreased with more $\mathrm{SrCO}_{3}$ incorporation in the glass phase.

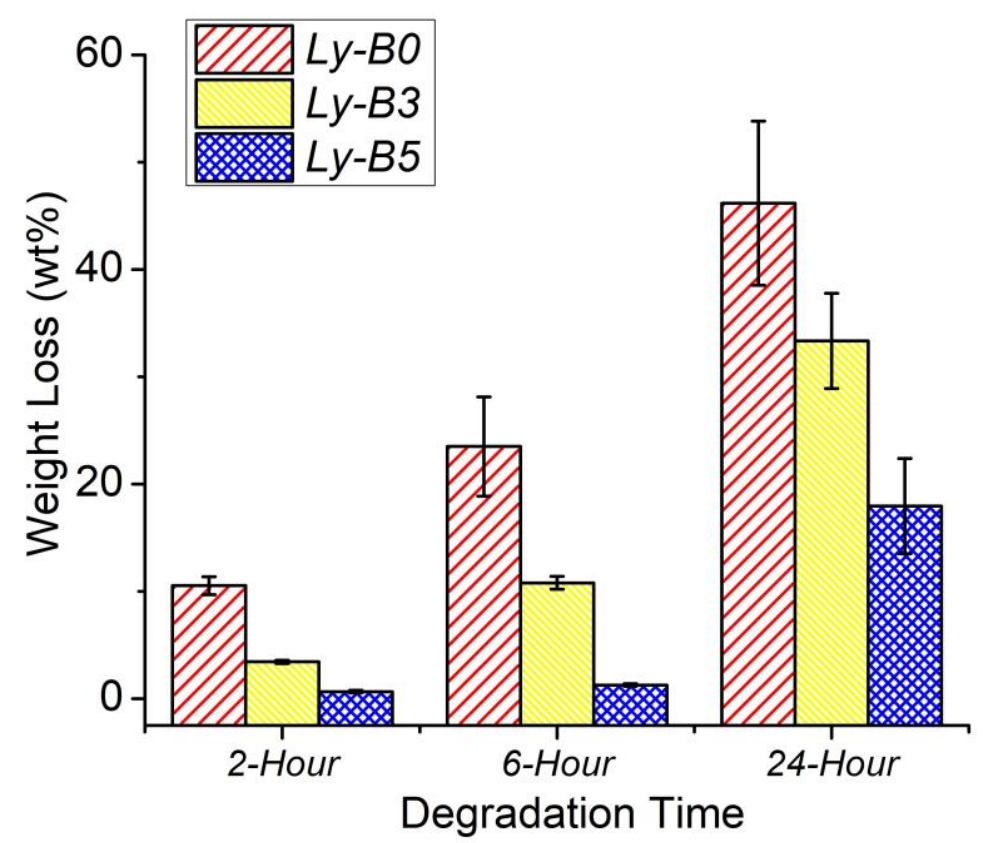

Figure 6.4. Weight loss of the coatings after different degradation times. The error bars show the standard deviation. The substrates used for the weight loss analysis were the as-received (not roughened) substrates.

The ion exchange ${ }^{190,191}$ between the glass network modifier cations and $\mathrm{H}^{+}$from water is the first step in the degradation of a glass in an aqueous environment, during which $\mathrm{H}^{+}$of the water molecule is donated to the non-bridging oxygen bonding with the modifier cation and the remaining $\mathrm{OH}^{-}$from the water molecule is freed causing increasing $\mathrm{pH}$ of the liquid ${ }^{192 .}$ This increase in $\mathrm{pH}$ has a positive influence on bioactivity as it inhibits the activity of osteoclasts reducing bone resorption ${ }^{192}$. $\mathrm{Na}^{+}$-water reaction usually happen first because of the initial enrichment of $\mathrm{Na}^{+}$on the glass surface ${ }^{191,193}$. Tilocca et al. ${ }^{191}$ investigated the water-bioglass interface by initio molecular dynamic simulations and found that $\mathrm{Ca}^{2+}$-water interaction mainly occurs after the dominant fraction of $\mathrm{Na}^{+}$is released into the solution. Then, additional modifier cations migrate from the glass bulk to the surface to react with water. The ion migration can be assisted by correlated forward-backward motion of an ion, during which the ion moves to an intermediate position and then returns to its initial site after another ion passing through ${ }^{193}$. The forward-backward movement of a modifier cation in the glass matrix involves a change in its coordination, where a number of bonds between the modifier ion and oxygen (M-O) have to be 
broken ${ }^{193}$ Since the strength of the ionic Sr-O bonding is relatively high, which is higher than those of alkali ions, such as $\mathrm{Na}^{+}$and $\mathrm{K}^{+}$, because divalent ions provide higher coordination numbers with oxygen atoms ${ }^{194}$. Therefore, it is difficult for $\mathrm{Sr}^{2+}$ to provide such 'transient sites' ${ }^{193}$. As a result, $\mathrm{Sr}^{2+}$ might block the pathway of other cations and then retarded the movement of other dissolution products, decreasing solubility of the glass coating/Ti6A14V substrate system.

Other than chemical composition, the residual stresses in glass coatings can also promote their degradation. The concentrations of the dissolution products, i.e. the degradation rate, can be influenced by the sign (tensile or compressive) or magnitude of residual stresses ${ }^{195,196}$ i.e. presence of tensile residual stresses in the coating can promote its dissolution rate, and the solubility of the coating can be hindered by a compressive residual stress ${ }^{197,198}$. Therefore, the compressive nature of the residual stresses in un-degraded Ly-B5 coatings (reported in Section 5.3.3 ), when Ly-B0 and Ly-B3 coatings undergo tensile residual stresses ${ }^{168}$, can also contribute to its slower dissolution rate (Figure 6.4).

A previous study ${ }^{120}$ revealed that the weight loss of powder discs $(2.2 \times \phi 6.4 \mathrm{~mm}$, $)$ of $L y$ $B 0, L y-B 3$ and $L y$-B5 after 24-hours incubation were $\sim 35.5 \%, \sim 5.75 \%$ and $\sim 3.42 \%$ respectively, which were significantly lower than those of the glass coatings after 24-hours incubation (Figure 6.4). Since weight loss of the glasses resulted from their degradation in de-ionized water 98 glass coatings degrade more intensely in the first 24 hours than the glasses discs. The greater surface area ${ }^{199,200}$ of the coatings can be one explanation of their faster degradation. Additionally, the residual stress in the glasses coatings, which influences their chemical potential, can also promote the degradation of glass coatings ${ }^{195,196}$

\subsubsection{Coating thickness}

Before the degradation, the average and standard deviation of the three measured thickness profiles were in the range $95-105 \mu \mathrm{m}$, and $4 \mu \mathrm{m}$ to $6 \mu \mathrm{m}$, respectively, for all of the coatings. The average and standard deviation of the thickness reduction due to degradation is illustrated in Figure 6.5. The substrates used for the thickness change analysis (Figure 6.5) were the as-received (not roughened) substrates. 


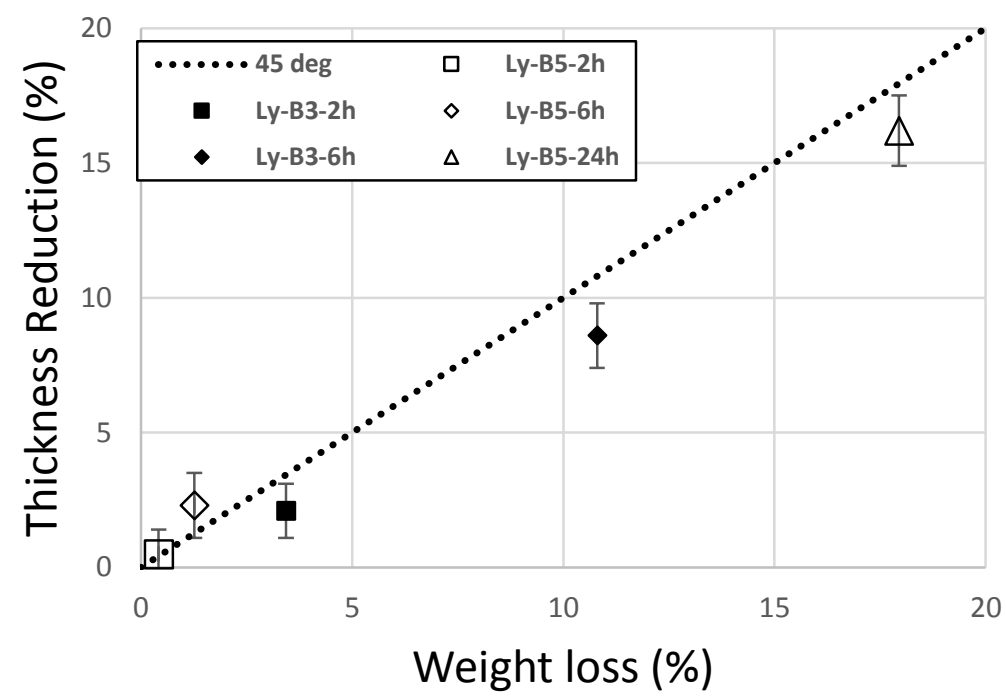

Figure 6.5. The thickness reduction (\%) due to degradation for Ly-B3 and Ly-B5 coatings in terms of weight loss (\%). The data points related to $\mathrm{Ly}-\mathrm{BO}(2 \mathrm{~h}, 6 \mathrm{~h}$ and $24 \mathrm{~h})$ and $\mathrm{Ly}-\mathrm{B3}(24 \mathrm{~h})$ data points are not shown here as these coating delaminated due to degradation even before any measurements could be done. The substrates used for the thickness change analysis were the as-received (not roughened) substrates.

The percentage of thickness reduction in all cases was relatively close to the percentage weight loss, indicating that degradation most likely occurs through a layer-by-layer removal of the glass from the open face of the coating. The data related to Ly-B0 (2h, 6h and 24h) and Ly-B3 (24h) samples are not shown in Figure 6.5 because these coating delaminated due to degradation even before any measurements could be performed. Figure 6.6 illustrates an Ly-B0 sample which delaminated after $2 \mathrm{~h}$ degradation.

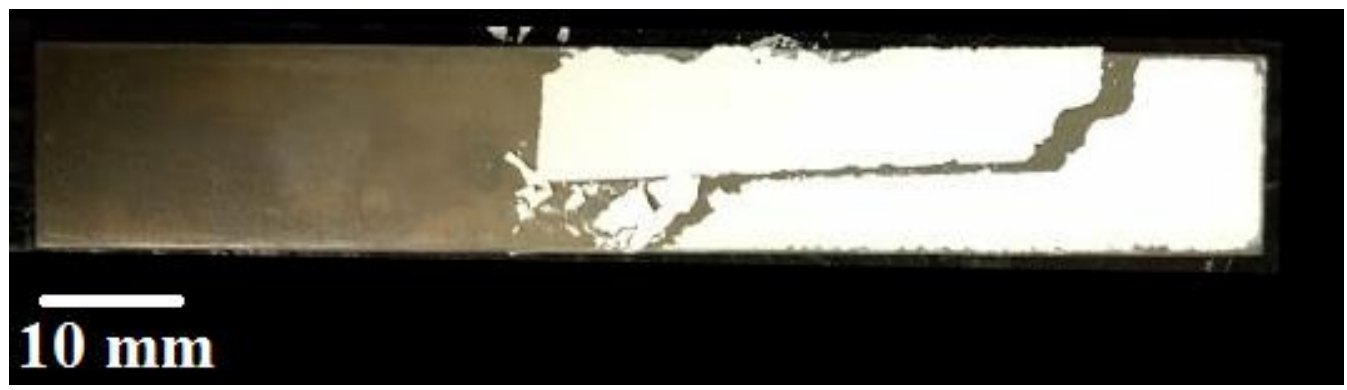

Figure 6.6. Ly-B0 sample delaminated after $2 \mathrm{~h}$ degradation. 


\subsubsection{Critical Mode I and Mode II strain energy release rate}

The mode I tests allowed multiple $G_{I C}$ measurements on a single specimen. This was not possible for the mode II tests because creating the mode I pre-crack left little room for the propagation of mode II crack and therefore only a single crack extension event could be recorded per test. Figure 6.7 illustrates the measured $G_{I C}$ and $G_{I I C}$ as a function of weight loss $\%$ for specimens made of different glass compositions. The substrates used for the fracture tests (Figure 6.7) were the 'as-received' (not roughened) substrates.

As shown in Figure 6.7, $G_{I C}$ and $G_{I I C}$ values of the glass coating/Ti6Al4V substrate systems for all glass compositions significantly decreased with increased degradation. As an example, for the Ly-B5/Ti6Al4V system, $G_{I C}$ and $G_{I I C}$ decreased by $80 \%$ and $85 \%$, respectively, after $17 \%$ of weight loss. The decrease in the $G_{I C}$ and $G_{I I C}$ of the system due to degradation was even more drastic for the Ly-B0 and Ly-B3 specimens, where after only $10 \%$ and 33\% weight loss, respectively, the coating delaminated upon removal from the deionized water even before it was tested (Figure 6.6). Figure 6.7 also shows that the critical strain energy release rate of glass/substrate systems under mode II loading is 3-4 times larger than in Mode I. This was expected as most materials show a higher resistance to crack under mode II loading condition, compared to mode I ${ }^{180,181}$. Finally, the Ly-B5/Ti6Al4V system which shows the highest $G_{I C}$ and $G_{I I C}$ before degradation among the three coating/substrate systems proved also to be the toughest system at any given level of degradation. 


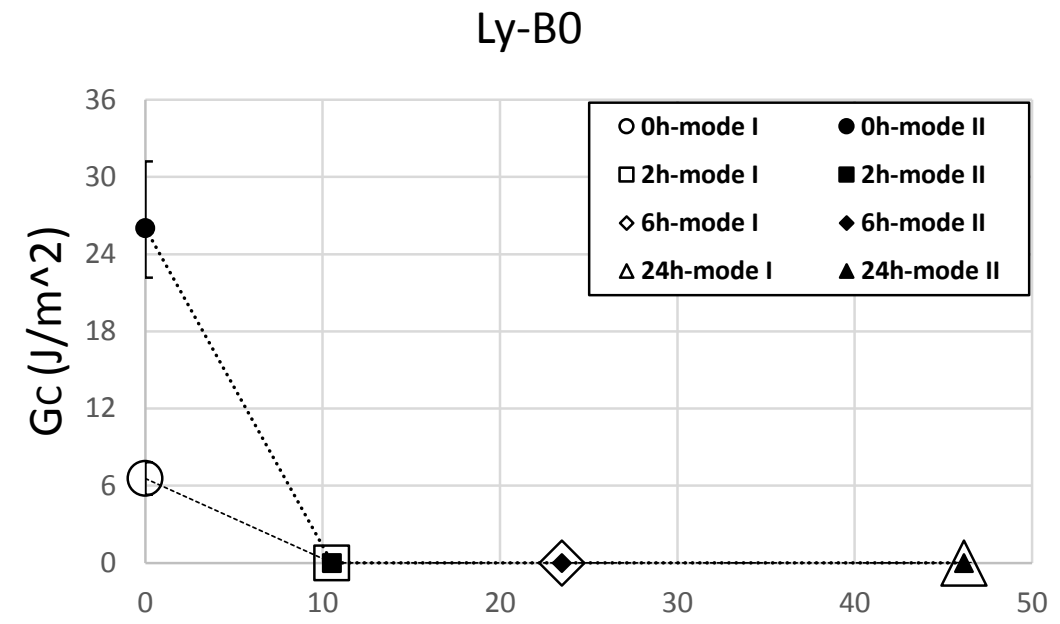

(a) Weight loss (\%)

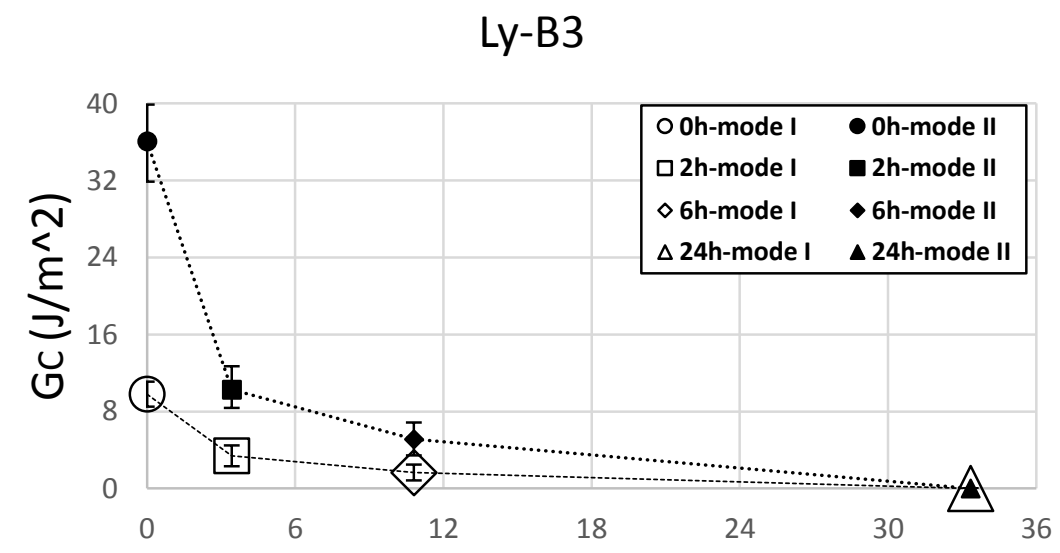

(b) Weight loss (\%) 
Ly-B5

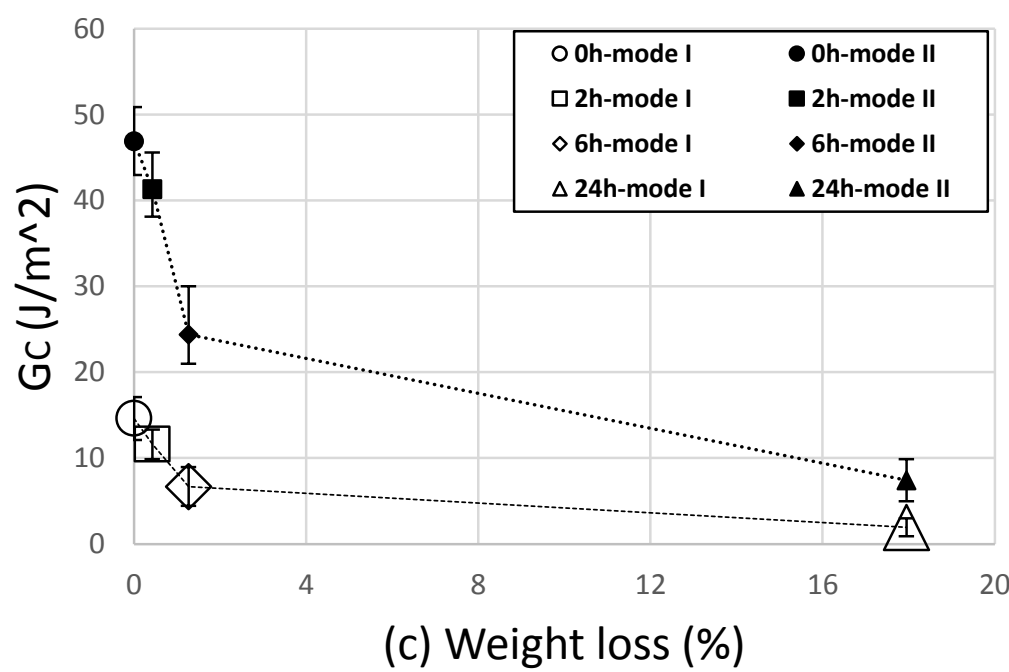

Figure 6.7. Critical strain energy release rate for coating/substrate systems made of Ly-B0 (a), LyB3 (b), Ly-B5 (c) in mode I and mode II loading condition. The data for undegraded samples (0h) is from Section 3.3.6 and Section 5.3.4 and the error bars illustrate the standard deviation for mode I tests and, min and max for mode II test results. Since Ly-BO (2h, $6 \mathrm{~h}$ and $24 \mathrm{~h}$ ) and Ly-B3 (24h) coating delaminated prior to fracture testing, it was assumed that their $G_{I C}=0$ and $G_{I I C}=0$. The substrates used for the fracture tests were the as-received (not roughened) substrates. The dashed lines are to help guide the eye.

Figure 6.8 shows the thickness of the residual glass on the substrate after fracture testing, measured using the techniques described in Section 6.2.2.5 . For both mode I and mode II, the residual glass thickness decreased with increasing degradation time indicating that the crack grew closer to the Ti6Al4V/glass interface when degraded. A similar trend was observed for degradable adhesive joints by Ameli et al. ${ }^{201}$ who reported a significant decrease in the thickness of the residual layer, due to degradation. They indicated that because of the significant reduction in the critical strain energy release rate after degradation, the chance of initiating microcracks across the bondline is lower and therefore the crack tends to propagate closer to more highly-strained arm, i.e., the interface between the coating and substrate. 


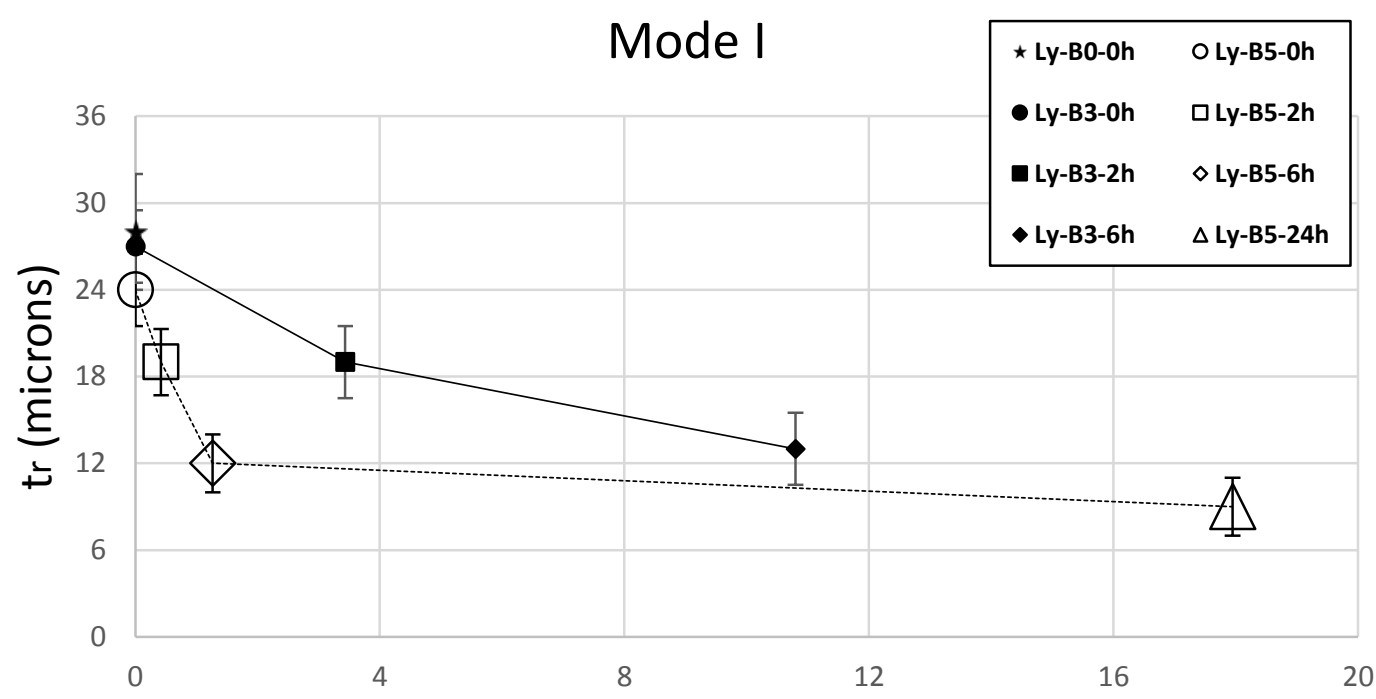

(a) Weight loss (\%)

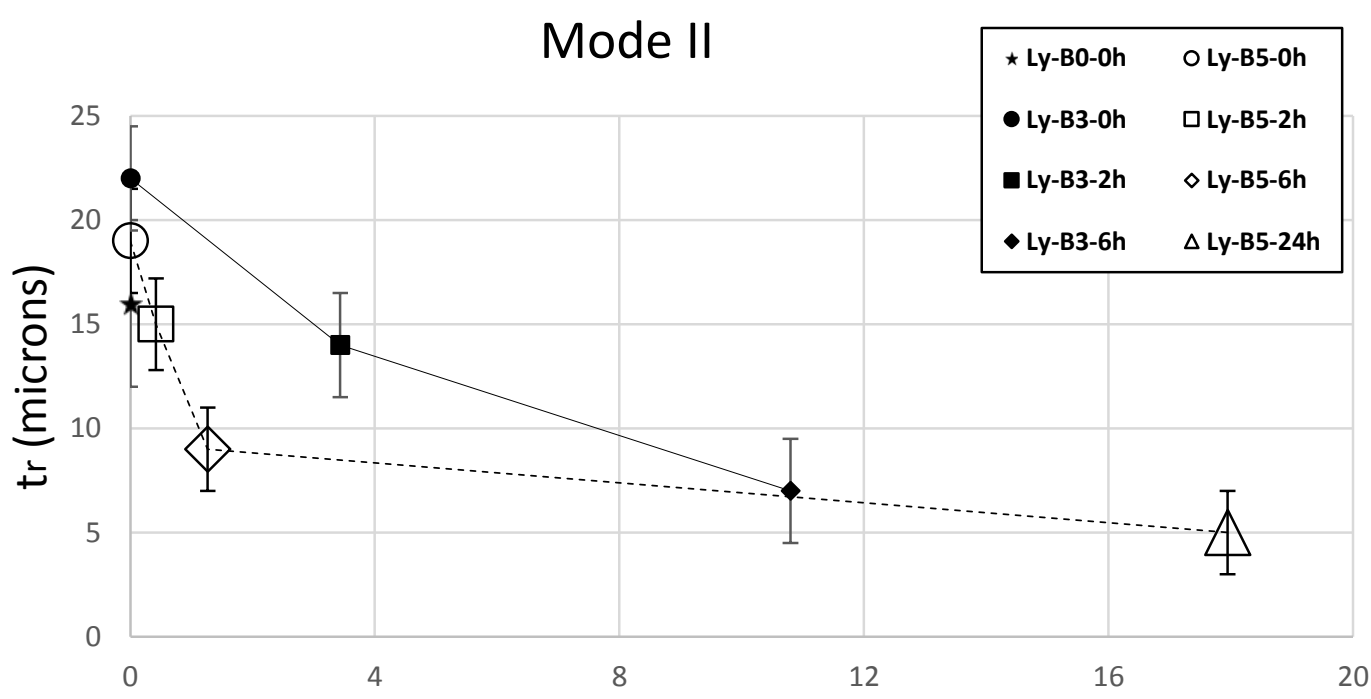

(b) Weight loss (\%)

Figure 6.8. The thickness of the residual layer, $t_{r}$, for mode I (a) and mode II (b) samples. The error bars illustrate the min and the max for the $t_{r}$ results. The substrates used for the fracture tests were the as-received (not roughened) substrates. The lines are to help guide the eye. 


\subsubsection{Effect of roughness on $G_{I C}$ and $G_{I I C}$ of the degraded samples}

Using the abrasive jet roughening procedure described in Section 6.2.2.6, the overall average roughness, $R a$, of the substrates increased from the as-received roughness $(R a)_{0}=0.5 \pm$ $0.05 \mu \mathrm{m}$ to $(R a)_{1}=1.4 \pm 0.3 \mu \mathrm{m}$ and $(R a)_{2}=3.1 \pm 0.4 \mu \mathrm{m}$. The coatings enamelled on the roughened substrates were degraded and tested as per Section 6.2.2.3 and 6.2.2.5, respectively.

As discussed in Section 6.3.5, some of the coatings delaminated immediately upon removal from the deionized water even at the minimum utilized degradation time. It was of interest to investigate whether an increased substrate roughness would increase the degradation time required for the coatings to delaminate in this manner. The Ly-B0 (2h), Ly-B3 (24h) and Ly-B5 (3 days) specimens were thus recreated using the roughened substrates, but creating the modified DCB required for testing still proved impossible. The coating lost its cohesion, and the glass powder making up the coating became loose and mixed in with the epoxy as it was being applied on the coating surface. Therefore, it was concluded that increasing the substrate roughness could not extend the maximum possible testable degradation period because the coating itself lost its coherence. This was somewhat expected as borate-based bioactive glasses are reported to have high degradation rate ${ }^{119}$ which leads to rapid reduction in their cohesion.

It was also of interest to investigate whether increasing the roughness of the substrates could increase the $G_{I C}$ and $G_{I I C}$ of the specimens that did not immediately delaminate upon removal from the deionized water that were tested in Section 6.3.5. This was motivated by the literature for adhesive joints, where it was found that increasing the roughness could increase the system toughness, provided that the crack propagation is close enough to the interface ${ }^{202,203}$. Azari et al. 202 found that the fatigue threshold strain energy release rate of adhesive joints increased with substrate roughnesses in the range of $1.3 \mu \mathrm{m}<R a<3.9 \mu \mathrm{m}$ due to crack growth retardation caused by crack path deflection around asperities. They observed that this increasing trend plateaued when $3.9 \mu m<R a<6.4 \mu m$ and then declined for very rough surfaces $(6.4 \mu m<$ $R a$ ), due to void formation and stress concentration at the roughness peaks. Prolongo et al. ${ }^{203}$ also reported a similar behaviour for adhesive joints, suggesting that there is an optimum value of roughness at which the adhesive exhibits maximum strength. They mentioned that this optimum roughness would be different for different epoxies. In order to investigate this phenomenon, the 
Ly-B3 (2h, 6h) and Ly-B5 (2h, 6h, 24h) specimens (which had near-interfacial failure during the fracture tests as shown in Figure 6.8) were recreated using the roughened substrates, and tested under mode I and mode II conditions as per Section 6.2.2.5 . Unfortunately, no significant difference in the measured $G_{C}$ or the crack path was observed. The crack path after degradation for all cases that could be tested, remained near interfacial, through the glass, rather than at the glass/metal interface. This suggests that the fracture toughness of the system as a whole was limited by the cohesive toughness of the coating itself, and roughening in the range studied here $(0.5 \mu m<R a<3.1 \mu m)$, was inconsequential in increasing the toughness of the system.

\subsection{Chapter Summary}

In this chapter, the mechanical performances of three borate-based bioactive glass coatings with increasing amounts of incorporated $\mathrm{SrCO}_{3}(0,15 \mathrm{~mol} \%$ and $25 \mathrm{~mol} \%$ respectively at the expense of $\mathrm{B}_{2} \mathrm{O}_{3}$ ) were investigated. The glass powders were enamelled onto Ti6Al4V and the coated samples were immersed in de-ionized water and incubated for 2, 6 and 24 hours. The weight loss of each glass coating composition was measured and it was found that the dissolution rate significantly decreased with increasing amounts of incorporated $\mathrm{SrCO}_{3}$. The thickness reduction due to degradation in all cases was relatively close to the percentage of weight loss, indicating that degradation occurred through a layer-by-layer removal of the glass from the open face of the coating. It was also found that residual stresses in the glass coatings can promote their degradation if they are tensile, and hinder their degradation if they are compressive. After drying the samples, bilayer double cantilever beam (DCB) specimens were created and tested to measure the effect of degradation on the fracture toughness of these glass/substrate systems. The DCB specimens were tested under mode I, opening, and mode II, shearing, loading conditions and $G_{I C}$ and $G_{I I C}$, the critical mode I and mode II strain energy release rates of the coating/substrate system, were calculated. It was found that the toughest coating/substrate system i.e. composed of the glass with $25 \mathrm{~mol}^{2} \mathrm{SrCO}_{3}$, lost $80 \%$ and $85 \%$ of its $G_{I C}$ and $G_{I I C}$, respectively, in less than $24 \mathrm{~h}$ of degradation. The drop in $G_{I C}$ and $G_{I I C}$ happened even more rapidly for other coating/substrate systems. Therefore, degradation significantly deteriorates the fracture toughness of borate bioactive glass coatings on Ti6A4V substrates. The glass coatings were enamelled on roughened substrates and 
tested to investigate whether increasing the roughness increases $G_{I C}$ and $G_{I I C}$ of the system. However, the crack path after degradation for all cases that could be tested, remained nearinterfacial rather than at the glass/metal interface, suggesting that the fracture toughness of the system as a whole was limited by the cohesive toughness of the glass itself, and roughening ( 0.5 $\mu \mathrm{m}<R a<3.1 \mu \mathrm{m}$ ), could not increase the toughness of the system. 


\section{Conclusions and future work}




\subsection{Summary}

In this thesis, a rigorous fracture mechanics study was performed on bioactive glass coatings for metallic substrates. The background, motivation and literature review were all covered in Chapter 1. Chapter 2 introduced the nomenclatures and compositions of the glasses used in this dissertation, as well as the process of manufacturing them. It also presented the procedure of coating the substrates with the manufactured glass. In Chapter 3 a novel methodology for quantifying the mode I critical strain energy release rate of enamelled bioactive glass coatings on Ti6A14V substrates was presented, inspired in part by methods used in the fracture mechanics of structural adhesive joints. A method for applying a uniform thickness bioactive glass coating on Ti6Al4V substrate was developed and used with bi-layer double cantilever beam (DCB) specimens in order to measure the critical mode I interfacial or bioactive glass critical strain energy release rate $\left(G_{I C}\right)$. This testing methodology was used to investigate the effect of glass coating's thickness on the $G_{I C}$ of the coating/substrate system. Chapter 4 further characterized the mechanics of bioactive glass coatings by characterizing silica-based and borate-based glasses in terms of their mechanical properties relevant to their use as metallic coating materials. In this chapter the mechanical performances of these two types of glass coatings were compared using the developed testing methodology of Chapter 3. In Chapter 5, another fracture mechanics based testing methodology was presented, this time to measure the mode II critical strain energy release rate $\left(G_{I I C}\right)$ of a series of borate-based bioactive glass coatings on Ti6Al4V substrates. Finally, Chapter 6 adapted the fracture mechanics based methodologies presented in Chapters 3 and 5 to investigate the effect of degradation on the resulting mode I and II critical strain energy release rates.

\subsection{Conclusions}

1. The first primary objective was to investigate the effect of glass coating thickness on the fracture of the coating/substrate system under mode I loading condition. Completing this objective lead to the following findings:

i. The mode I critical strain energy release rate of the coating/substrate system decreased significantly with coating thickness, i.e., thinner glass coatings proved to have higher resistance against fracture than thicker ones. This was found to be due to differences in 
the residual stresses in the coating formed as a result of the thermal mismatch between the coating and the substrate.

ii. Increases in the coating thickness were found to lead to higher residual stresses that tended to increase the crack driving force.

iii. If the coating thickness was sufficiently large, the residual stresses were sufficient to crack the glass layer, even prior to applying an external load. The critical thickness was calculated using existing fracture mechanics analyses, and found to be close to that measured experimentally, further confirming the efficacy of the fracture mechanics approach.

iv. The stability of the crack propagation within the coating was quantified by calculating the T-stress, and it was demonstrated that as the coating thickness increased, the crack destabilized and tended to kink rather than travel in a straight line.

2. The second primary objective was to investigate the effect of glass composition on the fracture of the coating/substrate system. Completing this objective lead to the following finding:

i. Borate-based glasses exhibited CTE that were closer to the substrate's (Ti6Al4V) CTE, a common alloy used in medical implants; this translated into higher mode I critical energy release rates for the borate-based glasses and lower residual stresses at the coating/substrate interface, outperforming the silica-based glass counterparts.

3. The third primary objective was to investigate the effect of loading condition on the fracture of the system. Completing this objective lead to the following findings:

i. Applying the mode I and mode II fracture testing methodology on a series of boratebased glass coating led to the conclusion that the measured $G_{I I c}$ values were 3 to 4 times their $G_{I C}$ counterparts.

ii. The distribution of residual stresses in the coatings were found to distribute uniformly over the cross section of the layer due to its relatively low thickness.

iii. Since the CTE's of borate glasses are generally close to that of Ti6Al4V, the crack driving force caused by the residual stresses was found to be negligible compared to that caused by the mechanical loading, indicating that the measured $G_{c}$ was very close to the intrinsic fracture toughness of the system.

iv. The mode II loading drove the mode I pre-crack towards the glass/substrate interface while the negative T-stress ensured that the deviation occurred at a small angle. The 
crack remained cohesive for all specimens, indicating that the bulk glass fracture toughness was lower than that of the interface.

4. The fourth primary objective was to investigate the effect of degradation on the fracture of the system. Completing this objective lead to the following findings:

i. Higher tensile (or conversely, compressive) residual stresses correlate with lower (or conversely, higher) degradation rates.

ii. The toughest coating/substrate system i.e. composed of the glass with $25 \mathrm{~mol} \% \mathrm{SrCO}_{3}$, lost $80 \%$ and $85 \%$ of its $G_{I C}$ and $G_{I I C}$, respectively, in less than $24 \mathrm{~h}$ of degradation.

iii. The drop in $G_{I C}$ and $G_{I I C}$ due to degradation occurred even more rapidly for other coating/substrate systems, suggesting that degradation significantly deteriorated the fracture toughness of borate bioactive glass coatings on Ti6A4V substrates.

5. The fifth primary objective was to investigate the effect of degradation on the fracture of the system. Completing this objective lead to the following finding:

i. Fracture toughness of the system as a whole was limited by the cohesive toughness of the glass itself, and roughening $(0.5 \mu \mathrm{m}<R a<3.1 \mu \mathrm{m})$ of the substrate could not increase the toughness of the system.

\subsection{Novel contributions}

The novel contributions of this dissertation can be summarized as:

i. For the first time, a novel methodology for quantifying the mode I critical strain energy release rate of bioactive glass coating on metallic substrates was developed, inspired in part by methods used in the fracture mechanics of structural adhesive joints. The methodology showed great promise in providing a more quantitative measure of bioactive glass coating adhesive/cohesive strength than the more common scratch or indentation tests.

ii. A method for applying a uniform thickness glass coating on Ti6Al4V substrate was developed that can be used with the developed fracture testing methodology to measure the critical strain energy release rate of the system. 
iii. The magnitude and distribution of residual stresses in the glass coating was measured for the first time and their effect on the fracture of the coating/substrate system was quantified.

iv. The effect of coating thickness on the fracture toughness of the system was quantified and practical recommendations regarding the optimum thickness of glass coating were given.

v. Knowing that the coatings on biomedical implants such as hip and knee prostheses are usually subjected to a combination of both shearing (mode II) and opening (mode I) loads, the mode II fracture testing methodology that was developed elucidated mechanical performance of bioactive glass coatings under mode II which was not previously quantified in the literature.

vi. For the first time, the effect of degradation on the toughness of glass/substrate system was quantitatively determined and the correlation between the residual stresses in the coating and their dissolution rate was studied. 


\subsection{Future work}

i. Investigating the effect of impact forces: During total hip replacement surgery, the surgeon is required to hammer the implant into the bone to locate the stem. This process might require multiple strikes with relatively high impact forces which can potentially crack or fracture the coating. Therefore, investigating the effect of such impact forces on the performance of the coating/substrate system can be a topic for further studies.

ii. Developing a finite element model to simulate in vivo loading: The mode I and mode II critical strain energy release rates of the coating/substrate system were quantified in this dissertation. This information can be fed in to a finite element model of the coated implant with the correct geometry in order to investigate whether the coating can survive in vivo loading conditions.

iii. Degradation study in simulated body fluid (SBF): such a fluid can be used as an alternative to deionized water as it better matches the composition and $\mathrm{pH}$ of human blood. Similar types of testing as in Chapter 5 can be done on the degraded samples to investigate whether the samples degraded in SBF exhibit different mechanical behaviour compared to those degraded in deionized water.

iv. Testing the fracture toughness of the wet specimens: The procedure proposed in Chapter 5 for measuring the toughness of the degraded specimens is based on testing the samples after they are removed from the water and completely dried, whereas in real applications, the coated implant would remain inside the human body fluid the entire time. Therefore, testing the adhesive/cohesive strength of the sample as it is wet (or maybe even while immersed) will provide a more realistic measure of the coating performance. 


\section{Appendix}




\section{Appendix-The effect of the compliance of the epoxy and coating layer on the measured $G_{I C}$ values}

As discussed in section 3.2.5.4, Equation (3.4) used for calculating the mode I critical strain energy release rate neglects the effect of the compliance of the epoxy layer and coating on the overall compliance of the bilayer DCB specimen. In order to investigate this effect on the calculated $G_{I C}$ values, the spring method of Troczynski and Camire ${ }^{139}$ was applied which models the DCB specimen as a beam on elastic foundation (Figure A.1(a)). This approach is based on the assumption that the compliance of a bilayer DCB specimen can be analyzed by studying two identical half-specimens each composed of a substrate, and a layer of epoxy and coating each with half of their actual thicknesses as shown in (Figure A.1(b)). This model has been reported to be in good agreement with the experimental data extracted from testing a series of steel arms joined with epoxy $^{139}$.

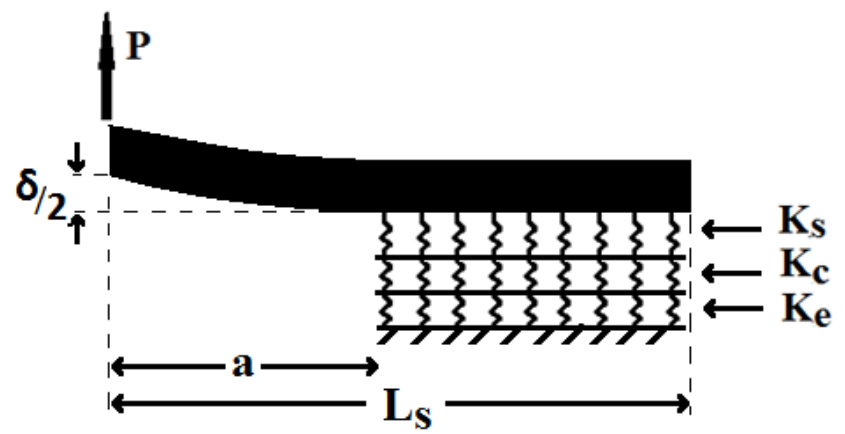

(a)

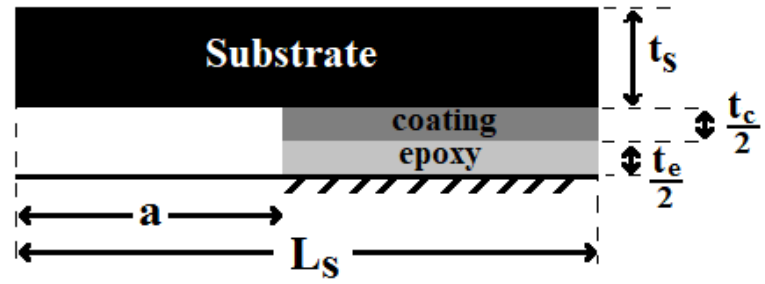

(b)

Figure A.1. Spring model of the foundation of a half bilayer DCB specimen developed by Troczynski and Camire ${ }^{139}$ (a). A half-specimen in this model is composed of one substrate, and a layer of epoxy and coating each with half of their actual thicknesses (b).

The total stiffness of the foundation of the upper (or lower) half of the specimen, $K_{t}$, can be written as:

$$
\frac{1}{K_{t}}=\frac{1}{K_{S}}+\frac{1}{K_{c}}+\frac{1}{K_{e}}
$$

where $K$ is the contributions of each layer to the stiffness of the foundation of the half-specimen, respectively, and subscripts $s, c$ and $e$ refer to the substrate, coating and epoxy, respectively. The parameters $K_{s}, K_{c}$ and $K_{e}$ can be calculated as 


$$
K_{s}=\frac{2 E_{s} w}{t_{s}}, K_{c}=\frac{4 E_{c} w}{t_{c}}, K_{e}=\frac{4 E_{e} w}{t_{e}}
$$

in which $E, w$ and $t$ are Young's modulus, width and thickness of the layers, respectively. The Young's modulus of the substrate, coating and epoxy were taken as $110 \mathrm{GPa}, 35 \mathrm{GPa}$ and $4 \mathrm{GPa}$, respectively and the width of specimen was $12.7 \mathrm{~mm}$. The thicknesses of the substrate and epoxy were 3.2 and $0.2 \mathrm{~mm}$, respectively and the coating thickness was chosen from Table 3.1.

Since the lower and upper half of the specimen are identical, they will equally contribute to the overall compliance of the system. Therefore, the total compliance will be double the compliance of each half-specimen, which according to Troczynski and Camire ${ }^{139}$ can be found as

$$
C=\frac{\delta}{P}=2\left[\frac{a^{3}}{3 E_{s} I_{t}}+\frac{A a}{2 E_{s} I_{t} \lambda^{2}}+\frac{B}{2 E_{s} I_{t} \lambda^{3}}\right]
$$

where $\delta$ is the deflection of adherend, $P$ is the applied load, $a$ is the crack length and $I_{t}$ is the second moment of area of the cross section of upper (or lower) half of the system with respect to the neutral axis. The parameter $\lambda^{4}=\frac{K_{t}}{4 E_{s} I_{t}}$, and functions $A$ and $B$ in Equation (A.3) are defined as 139

$$
\begin{gathered}
A=\frac{S_{1}}{S_{2}}+\frac{2 a \lambda\left(S_{3}-S_{4}\right)}{S_{2}}, B=\frac{a \lambda S_{1}}{S_{2}}+\frac{S_{3}-S_{4}}{S_{2}} \\
S_{1}=\sinh ^{2}\left(\lambda\left(L_{s}-a\right)\right)+\sin ^{2}\left(\lambda\left(L_{s}-a\right)\right) \\
S_{2}=\sinh ^{2}\left(\lambda\left(L_{s}-a\right)\right)-\sin ^{2}\left(\lambda\left(L_{s}-a\right)\right) \\
S_{3}=\sinh \left(\lambda\left(L_{s}-a\right)\right) \times \cosh \left(\lambda\left(L_{s}-a\right)\right) \\
S_{4}=\sin \left(\lambda\left(L_{s}-a\right)\right) \times \cos \left(\lambda\left(L_{s}-a\right)\right)
\end{gathered}
$$

and $L_{s}$ is the length of the specimen (Figure A.1). The strain energy release rate $G$ was be calculated using 139

$$
G=\left(\frac{P^{2}}{2 w}\right) \frac{d C}{d a}
$$


Equation A.4 was used to find the mode I critical strain energy release rate of all the samples in Table 3.1 and it was found that the error in $G_{I C}$ introduced by neglecting the compliance of the epoxy and glass layers was in all cases $<10 \%$. 


\section{References}

1. Gomez-Vega, J. M., Saiz, E., Tomsia, A. P., Marshall, G. W. \& Marshall, S. J. Bioactive glass coatings with hydroxyapatite and Bioglass particles on Ti-based implants. 1.

Processing. Biomaterials 21, 105-111 (2000).

2. Gomez-Vega, J. M. et al. Novel Bioactive Functionally Graded Coatings on Ti6Al4V. Adv. Mater. 12, 894-898 (2000).

3. Gross, S. \& Abel, E. W. A finite element analysis of hollow stemmed hip prostheses as a means of reducing stress shielding of the femur. J. Biomech. 34, 995-1003 (2001).

4. Wang, K. The use of titanium for medical applications in the USA. Mater. Sci. Eng. A 213, 134-137 (1996).

5. Peddi, L., Brow, R. K. \& Brown, R. F. Bioactive borate glass coatings for titanium alloys. J. Mater. Sci. Mater. Med. 19, 3145-3152 (2008).

6. Burg, K. J. L., Porter, S. \& Kellam, J. F. Biomaterial developments for bone tissue engineering. Biomaterials 21, 2347-2359 (2000).

7. HIp Replacement Surgery - Pain, Relief, Recovery, Arthroplasty. Available at: http://www.orthopedicsurgerybook.com/hip-replacement-surgery-pain-resurfacing.php. (Accessed: 27th November 2017)

8. APS Materials, Inc. - Biomedical | Biomedical Implants | Coatings. APS Materials, Inc. Available at: http://apsmaterials.com/biomedical/. (Accessed: 27th November 2017)

9. Gomez-Vega, J. M., Saiz, E., Tomsia, A. P., Marshall, G. W. \& Marshall, S. J. Bioactive glass coatings with hydroxyapatite and Bioglass particles on Ti-based implants. 1. Processing. Biomaterials 21, 105-111 (2000). 
10. Gomez-Vega, J. M., Saiz, E. \& Tomsia, A. P. Glass-based coatings for titanium implant alloys. J. Biomed. Mater. Res. 46, 549-559 (1999).

11. Geesink, R. G., de Groot, K. \& Klein, C. P. Bonding of bone to apatite-coated implants. J. Bone Joint Surg. Br. 70, 17-22 (1988).

12. Glocker, D. \& Ranade, S. Medical Coatings and Deposition Technologies. (John Wiley \& Sons, 2016).

13. Nakamura, S. et al. Thermal Expansion of Hydroxyapatite- $\beta$-Tricalcium Phosphate Ceramics. Thermochim. Acta 165, 57-72 (1990).

14. Oonishi, H. et al. The effect of hydroxyapatite coating on bone growth into porous titanium alloy implants. J. Bone Joint Surg. Br. 71, 213-216 (1989).

15. Ong, J. L., Carnes, D. L. \& Bessho, K. Evaluation of titanium plasma-sprayed and plasmasprayed hydroxyapatite implants in vivo. Biomaterials 25, 4601-4606 (2004).

16. Darimont, G. L., Cloots, R., Heinen, E., Seidel, L. \& Legrand, R. In vivo behaviour of hydroxyapatite coatings on titanium implants: a quantitative study in the rabbit. Biomaterials 23, 2569-2575 (2002).

17. Lu, Y.-P., Li, M.-S., Li, S.-T., Wang, Z.-G. \& Zhu, R.-F. Plasma-sprayed hydroxyapatite+titania composite bond coat for hydroxyapatite coating on titanium substrate. Biomaterials 25, 4393-4403 (2004).

18. Yang, Y.-C. \& Chang, E. Measurements of residual stresses in plasma-sprayed hydroxyapatite coatings on titanium alloy. Surf. Coat. Technol. 190, 122-131 (2005).

19. Hench, L. L., Splinter, R. J., Allen, W. C. \& Greenlee, T. K. Bonding mechanisms at the interface of ceramic prosthetic materials. J. Biomed. Mater. Res. 5, 117-141 (1971).

20. Hench, L. L. An Introduction to Bioceramics. (World Scientific, 1993). 
21. Coughlan, A., Boyd, D., Douglas, C. W. I. \& Towler, M. R. Antibacterial coatings for medical devices based on glass polyalkenoate cement chemistry. J. Mater. Sci. Mater. Med. 19, 3555-3560 (2008).

22. Coughlan, A., Boyd, D. \& Towler, M. R. Biofilm inhibitory coatings formulated from glass polyalkenoate cement chemistry: an evaluation of their adhesive nature. J. Mater. Sci. 44, 1652-1655 (2009).

23. Coughlan, A., Scanlon, K., Mahon, B. P. \& Towler, M. R. Zinc and silver glass polyalkenoate cements: an evaluation of their antibacterial nature. Biomed. Mater. Eng. 20, 99-106 (2010).

24. Coughlan, A., Ryan, M. P., Cummins, N. M. \& Towler, M. R. The response of Pseudomonas aeruginosa biofilm to the presence of a glass polyalkenoate cement formulated from a silver containing glass. J. Mater. Sci. 46, 285-287 (2010).

25. Lopez-Esteban, S. et al. Bioactive glass coatings for orthopedic metallic implants. J. Eur. Ceram. Soc. 23, 2921-2930 (2003).

26. Sola, A., Bellucci, D. \& Cannillo, V. Enamelled coatings produced with low-alkaline bioactive glasses. Surf. Coat. Technol. 248, 1-8 (2014).

27. Schrooten, J. \& Helsen, J. A. Adhesion of bioactive glass coating to Ti6Al4V oral implant. Biomaterials 21, 1461-1469 (2000).

28. Mehdipour, M., Afshar, A. \& Mohebali, M. Electrophoretic deposition of bioactive glass coating on 316L stainless steel and electrochemical behavior study. Appl. Surf. Sci. 258, 9832-9839 (2012).

29. Fiorilli, S. et al. Electrophoretic deposition of mesoporous bioactive glass on glass-ceramic foam scaffolds for bone tissue engineering. J. Mater. Sci. Mater. Med. 26, 21 (2015). 
30. Ponsot, I., Detsch, R., Boccaccini, A. R. \& Bernardo, E. Waste derived glass ceramic composites prepared by low temperature sintering/sinter-crystallisation. Adv. Appl. Ceram. 114, S17-S25 (2015).

31. Brow, R.K. \& Watkins, R.D. Reactions and bonding between glasses and titanium. in 2530 (ASME, 1987).

32. Saha, S. K., Jain, H., Goldstein, J., Miller, A. C. \& Brow, R. K. Reaction between titanium and B2O3 melt/glass. Phys. Chem. Glas. 39, 118-121 (1998).

33. Charles, L. F., Shaw, M. T., Olson, J. R. \& Wei, M. Fabrication and mechanical properties of PLLA/PCL/HA composites via a biomimetic, dip coating, and hot compression procedure. J. Mater. Sci. Mater. Med. 21, 1845-1854 (2010).

34. Kitsugi, T. et al. Bone-bonding behavior of plasma-sprayed coatings of BioglassR, AWglass ceramic, and tricalcium phosphate on titanium alloy. J. Biomed. Mater. Res. 30, 261269 (1996).

35. Tsui, Y. C., Doyle, C. \& Clyne, T. W. Plasma sprayed hydroxyapatite coatings on titanium substrates. Part 1: Mechanical properties and residual stress levels. Biomaterials 19, 20152029 (1998).

36. Sun, L., Berndt, C. C., Gross, K. A. \& Kucuk, A. Material fundamentals and clinical performance of plasma-sprayed hydroxyapatite coatings: a review. J. Biomed. Mater. Res. $58,570-592(2001)$.

37. Gross, K. A., Gross, V. \& Berndt, C. C. Thermal Analysis of Amorphous Phases in Hydroxyapatite Coatings. J. Am. Ceram. Soc. 81, 106-112 (1998). 
38. Wang, B. C., Chang, E., Lee, T. M. \& Yang, C. Y. Changes in phases and crystallinity of plasma-sprayed hydroxyapatite coatings under heat treatment: a quantitative study. $J$. Biomed. Mater. Res. 29, 1483-1492 (1995).

39. Cook, S. D., Thomas, K. A. \& Kay, J. F. Experimental coating defects in hydroxylapatitecoated implants. Clin. Orthop. 280-290 (1991).

40. Lin, J. C., Liu, M. L. \& Ju, C. P. Structure and properties of hydroxyapatite-bioactive glass composites plasma sprayed on Ti6Al4V. J. Mater. Sci. Mater. Med. 5, 279-283 (1994).

41. Chen, M., Li, W., Shen, M., Zhu, S. \& Wang, F. Glass coatings on stainless steels for hightemperature oxidation protection: Mechanisms. Corros. Sci. 82, 316-327 (2014).

42. Pavón, J. et al. Stress-corrosion cracking by indentation techniques of a glass coating on Ti6Al4V for biomedical applications. J. Eur. Ceram. Soc. 26, 1159-1169 (2006).

43. Lotfibakhshaiesh, N., Brauer, D. S. \& Hill, R. G. Bioactive glass engineered coatings for Ti6Al4V alloys: Influence of strontium substitution for calcium on sintering behaviour. $J$. Non-Cryst. Solids 356, 2583-2590 (2010).

44. Chalker, P. R., Bull, S. J. \& Rickerby, D. S. A review of the methods for the evaluation of coating-substrate adhesion. Mater. Sci. Eng. A 140, 583-592 (1991).

45. King, B. W., Tripp, H. P. \& Duckworth, W. H. Nature of Adherence of Porcelain Enamels to Metals. J. Am. Ceram. Soc. 42, 504-525 (1959).

46. Pask, J. A. \& Fulrath, R. M. Fundamentals of Glass-to-Metal Bonding: VIII, Nature of Wetting and Adherence. J. Am. Ceram. Soc. 45, 592-596 (1962).

47. Joseph A. Pask. From technology to the science of glass metal and ceramic metal sealing. Am. Ceram. Soc. Bull. (1987).

48. Adhesion science and engineering. (Elsevier, 2002). 
49. Irwin, G. Analysis of stresses and strains near the end of a crack traversing a plate. J. Appl. Mech. 24, 361-364 (1957).

50. Li, J. \& Beres, W. Scratch Test for Coating/Substrate Systems - A Literature Review. Can. Metall. Q. 46, 155-173 (2007).

51. ASTM C1624 Standard Test Method for Adhesion Strength and Mechanical Failure Modes of Ceramic Coatings by Quantitative Single Point Scratch Testing. (2015).

52. Examining Interfacial Adhesion of Biomedical Coatings. Available at: https://www.pcimag.com/articles/100920-examining-interfacial-adhesion-of-biomedicalcoatings. (Accessed: 11th October 2017)

53. Heavens, O. S. Some factors influencing the adhesion of films produced by vacuum evaporation. J Phys Radium 11, 355-360 (1950).

54. Laugier, M. An energy approach to the adhesion of coatings using the scratch test. Thin Solid Films 117, 243-249 (1984).

55. von Stebut, J., Rezakhanlou, R., Anoun, K., Michel, H. \& Gantois, M. Major damage mechanisms during scratch and wear testing of hard coatings on hard substrates. Thin Solid Films 181, 555-564 (1989).

56. Bromark, M., Larsson, M., Hedenqvist, P., Olsson, M. \& Hogmark, S. Influence of substrate surface topography on the critical normal force in scratch adhesion testing of TiNcoated steels. Surf. Coat. Technol. 52, 195-203 (1992).

57. Lardner, T. J., Ritter, J. E., Shiao, M. L. \& Lin, M. R. Behavior of indentation cracks near free surfaces and interfaces. Int. J. Fract. 44, 133-143 (1990). 
58. Anstis, G. r., Chantikul, P., Lawn, B. r. \& Marshall, D. b. A Critical Evaluation of Indentation Techniques for Measuring Fracture Toughness: I, Direct Crack Measurements. J. Am. Ceram. Soc. 64, 533-538 (1981).

59. Pharr, G. M. Measurement of mechanical properties by ultra-low load indentation. Mater. Sci. Eng. A 253, 151-159 (1998).

60. Fett, T., Burghard, Z., Zimmermann, A. \& Aldinger, F. Residual Stresses and Stress Intensity Factors for Vickers Indentation Cracks in Glass Derived from COD Measurements. Adv. Eng. Mater. 6, 914-918 (2004).

61. Lawn, B. R., Evans, A. G. \& Marshall, D. B. Elastic/Plastic Indentation Damage in Ceramics: The Median/Radial Crack System. J. Am. Ceram. Soc. 63, 574-581 (1980).

62. Bhattacharya, A. K., Petrovic, J. J. \& Danforth, S. C. Indentation method for determining the macroscopic fracture energy of brittle bimaterial interfaces. J. Am. Ceram. Soc. 75, 413-417 (1992).

63. Griffith, A. A. The Phenomena of Rupture and Flow in Solids. Philos. Trans. R. Soc. Lond. Math. Phys. Eng. Sci. 221, 163-198 (1921).

64. Broek, D. Elementary engineering fracture mechanics. (Springer Science \& Business Media, 1982).

65. Fett T. Computation of the crack opening displace- ments for Vickers indentation. (2002).

66. Fett, T., Kounga, A. B. \& Rödel, J. Stresses and stress intensity factor from COD of Vickers indentation cracks. J. Mater. Sci. 39, 2219-2221 (2004).

67. Kruzic, J. J., Kim, D. K., Koester, K. J. \& Ritchie, R. O. Indentation techniques for evaluating the fracture toughness of biomaterials and hard tissues. J. Mech. Behav. Biomed. Mater. 2, 384-395 (2009). 
68. Hsiung, C.-H. H. et al. Impact of doping on the mechanical properties of acicular mullite. $J$. Eur. Ceram. Soc. 33, 1955-1965 (2013).

69. Laugier, M. T. New formula for indentation toughness in ceramics. J. Mater. Sci. Lett. 6, 355-356 (1987).

70. Mercer, C., Williams, J. R., Clarke, D. R. \& Evans, A. G. On a ferroelastic mechanism governing the toughness of metastable tetragonal-prime $\left(\mathrm{t}^{\prime}\right)$ yttria-stabilized zirconia. Proc. R. Soc. Lond. Math. Phys. Eng. Sci. 463, 1393-1408 (2007).

71. Braem, A. et al. Bioactive glass-ceramic coated titanium implants prepared by electrophoretic deposition. Mater. Sci. Eng. C 32, 2267-2273 (2012).

72. ASTM International. ASTM F1147 Tension Testing for Calcium Phosphate and Metallic Coatings. in (2017).

73. Berndt, C. C. \& Lin, C. K. Measurement of adhesion for thermally sprayed materials. J. Adhes. Sci. Technol. 7, 1235-1264 (1993).

74. Baino, F. \& Vitale-Brovarone, C. Wollastonite-containing bioceramic coatings on alumina substrates: Design considerations and mechanical modelling. Ceram. Int. 41, 11464-11470 (2015).

75. Chen, Q., Baino, F., Pugno, N. M. \& Vitale-Brovarone, C. Bonding strength of glassceramic trabecular-like coatings to ceramic substrates for prosthetic applications. Mater. Sci. Eng. C Mater. Biol. Appl. 33, 1530-1538 (2013).

76. Cook, S. D. et al. Hydroxylapatite coating of porous implants improves bone ingrowth and interface attachment strength. J. Biomed. Mater. Res. 26, 989-1001 (1992). 
77. Hayashi, K., Inadome, T., Mashima, T. \& Sugioka, Y. Comparison of bone-implant interface shear strength of solid hydroxyapatite and hydroxyapatite-coated titanium implants. J. Biomed. Mater. Res. 27, 557-563 (1993).

78. Wang, B. C., Lee, T. M., Chang, E. \& Yang, C. Y. The shear strength and the failure mode of plasma-sprayed hydroxyapatite coating to bone: the effect of coating thickness. $J$. Biomed. Mater. Res. 27, 1315-1327 (1993).

79. De Groot, K., Geesink, R., Klein, C. P. a. T. \& Serekian, P. Plasma sprayed coatings of hydroxylapatite. J. Biomed. Mater. Res. 21, 1375-1381 (1987).

80. Thomas, K. A., Kay, J. F., Cook, S. D. \& Jarcho, M. The effect of surface macrotexture and hydroxylapatite coating on the mechanical strengths and histologic profiles of titanium implant materials. J. Biomed. Mater. Res. 21, 1395-1414 (1987).

81. Schrooten, J., Van Oosterwyck, H., Vander Sloten, J. \& Helsen, J. A. Adhesion of new bioactive glass coating. J. Biomed. Mater. Res. 44, 243-252 (1999).

82. Anderson, T. L. Fracture Mechanics: Fundamentals and Applications, Third Edition. (CRC Press, 2005).

83. Ameli, A., Papini, M., Schroeder, J. A. \& Spelt, J. K. Fracture R-curve characterization of toughened epoxy adhesives. Eng. Fract. Mech. 77, 521-534 (2010).

84. Nadimpalli, S. P. V. \& Spelt, J. K. R-curve behavior of $\mathrm{Cu}-\mathrm{Sn} 3.0 \mathrm{Ag} 0.5 \mathrm{Cu}$ solder joints: Effect of mode ratio and microstructure. Mater. Sci. Eng. A 527, 724-734 (2010).

85. Jindal, P. C., Quinto, D. T. \& Wolfe, G. J. Adhesion measurements of chemically vapor deposited and physically vapor deposited hard coatings on WC $\square$ Co substrates. Thin Solid Films 154, 361-375 (1987). 
86. Takadoum, J. \& Bennani, H. H. Influence of substrate roughness and coating thickness on adhesion, friction and wear of TiN films. Surf. Coat. Technol. 96, 272-282 (1997).

87. Malzbender, J. \& de With, G. Cracking and residual stress in hybrid coatings on float glass. Thin Solid Films 359, 210-214 (2000).

88. Schmidt, H. Inorganic-organic composites by sol-gel techniques. J. Sol-Gel Sci. Technol. 1, 217-231 (1994).

89. Chai, H. Bond Thickness Effect in Adhesive Joints and Its Significance for Mode I Interlaminar Fracture of Composites. in (ASTM International, 1984). doi:10.1520/STP35350S

90. Bascom, W. D., Cottington, R. L., Jones, R. L. \& Peyser, P. The fracture of epoxy- and elastomer-modified epoxy polymers in bulk and as adhesives. J. Appl. Polym. Sci. 19, 2545-2562 (1975).

91. Loch, A. J. K. \& Shaw, S. J. The Fracture Resistance of a Toughened Epoxy Adhesive. J. Adhes. 12, 59-77 (1981).

92. Pardoen, T., Ferracin, T., Landis, C. M. \& Delannay, F. Constraint effects in adhesive joint fracture. J. Mech. Phys. Solids 53, 1951-1983 (2005).

93. Azari, S., Papini, M. \& Spelt, J. K. Effect of adhesive thickness on fatigue and fracture of toughened epoxy joints - Part I: Experiments. Eng. Fract. Mech. 78, 153-162 (2011).

94. Zhao, Y., Song, M. \& Liu, J. Characteristics of bioactive glass coatings obtained by pulsed laser deposition. Surf. Interface Anal. 40, 1463-1468 (2008).

95. Hench, L. L., Hench, J. W. \& Greenspan, D. C. Bioglass: a short history and bibliography. J. Australas. Ceram. Soc. 40, 1-42 (2004). 
96. Hench, L. L. Bioceramics: From Concept to Clinic. J. Am. Ceram. Soc. 74, 1487-1510 (1991).

97. Hench, L. L. Bioceramics. J. Am. Ceram. Soc. 81, 1705-1728 (1998).

98. Huang, W., Day, D. E., Kittiratanapiboon, K. \& Rahaman, M. N. Kinetics and mechanisms of the conversion of silicate (45S5), borate, and borosilicate glasses to hydroxyapatite in dilute phosphate solutions. J. Mater. Sci. Mater. Med. 17, 583-596 (2006).

99. Han, X. \& Day, D. E. Reaction of sodium calcium borate glasses to form hydroxyapatite. $J$. Mater. Sci. Mater. Med. 18, 1837-1847 (2007).

100. Day, D. E., White, J. E., Brown, R. F. \& McMenamin, K. D. Transformation of borate glasses into biologically useful materials. Glass Technol. 44, 75-81 (2003).

101. Liang, W. et al. Bioactive borate glass scaffold for bone tissue engineering. J. Non-Cryst. Solids 354, 1690-1696 (2008).

102. Xiao, W. et al. Evaluation of Ti implants coated with Ag-containing borate bioactive glass for simultaneous eradication of infection and fracture fixation in a rabbit tibial model. $J$. Mater. Res. 27, 3147-3156 (2012).

103. Bellucci, D., Cannillo, V. \& Sola, A. Coefficient of thermal expansion of bioactive glasses: Available literature data and analytical equation estimates. Ceram. Int. 37, 2963-2972 (2011).

104. Pavón, J. J., Jiménez-Piqué, E., Anglada, M., Saiz, E. \& Tomsia, A. P. Fatigue behaviour of a glass coating on Ti6AL4V for biomedical applications. Rev. Fac. Ing. Univ. Antioquia 115-128 (2006). 
105. Huang, K. et al. Sol-gel derived mesoporous 58S bioactive glass coatings on AZ31 magnesium alloy and in vitro degradation behavior. Surf. Coat. Technol. 240, 137-144 (2014).

106. Yang, C. Y., Wang, B. C., Chang, E. \& Wu, B. C. Bond degradation at the plasma-sprayed HA coating/Ti-6AI-4V alloy interface: an in vitro study. J. Mater. Sci. Mater. Med. 6, 258265 (1995).

107. Kangasniemi, I. M. O., Verheyen, C. C. P. M., van der Velde, E. A. \& De Groot, K. In vivo tensile testing of fluorapatite and hydroxylapatite plasma-sprayed coatings. J. Biomed. Mater. Res. 28, 563-572 (1994).

108. Wylde, J. W. \& Spelt, J. K. Measurement of adhesive joint fracture properties as a function of environmental degradation. Int. J. Adhes. Adhes. 18, 237-246 (1998).

109. Ameli, A., Azari, S., Papini, M. \& Spelt, J. K. Characterization and prediction of fracture properties in hygrothermally degraded adhesive joints: an open-faced approach. J. Adhes. Sci. Technol. 27, 1080-1103 (2013).

110. Ameli, A., Papini, M. \& Spelt, J. K. Fracture R-curve of a toughened epoxy adhesive as a function of irreversible degradation. Mater. Sci. Eng. A 527, 5105-5114 (2010).

111. Ameli, A., Datla, N. V., Azari, S., Papini, M. \& Spelt, J. K. Prediction of environmental degradation of closed adhesive joints using data from open-faced specimens. Compos. Struct. 94, 779-786 (2012).

112. Ameli, A., Papini, M. \& Spelt, J. K. Hygrothermal degradation of two rubber-toughened epoxy adhesives: Application of open-faced fracture tests. Int. J. Adhes. Adhes. 31, 9-19 (2011). 
113. Datla, N. V., Ulicny, J., Carlson, B., Papini, M. \& Spelt, J. K. Mixed-mode fatigue behavior of degraded toughened epoxy adhesive joints. Int. J. Adhes. Adhes. 31, 88-96 (2011).

114. Kaur, G. et al. A review of bioactive glasses: Their structure, properties, fabrication and apatite formation. J. Biomed. Mater. Res. A 102, 254-274 (2014).

115. Huang, W., Day, D. E., Kittiratanapiboon, K. \& Rahaman, M. N. Kinetics and mechanisms of the conversion of silicate (45S5), borate, and borosilicate glasses to hydroxyapatite in dilute phosphate solutions. J. Mater. Sci. Mater. Med. 17, 583-596 (2006).

116. Yao, A. et al. In Vitro Bioactive Characteristics of Borate-Based Glasses with Controllable Degradation Behavior. J. Am. Ceram. Soc. 90, 303-306 (2007).

117. El-Ghannam, A., Ducheyne, P. \& Shapiro, I. M. Bioactive material template for in vitro, synthesis of bone. J. Biomed. Mater. Res. 29, 359-370 (1995).

118. Maeno, S. et al. The effect of calcium ion concentration on osteoblast viability, proliferation and differentiation in monolayer and 3D culture. Biomaterials 26, 4847-4855 (2005).

119. Brown, R. F. et al. Effect of borate glass composition on its conversion to hydroxyapatite and on the proliferation of MC3T3-E1 cells. J. Biomed. Mater. Res. A 88, 392-400 (2009).

120. Li, Y. et al. Antibacterial and osteo-stimulatory effects of a borate-based glass series doped with strontium ions. J. Biomater. Appl. 31, 674-683 (2016).

121. Li, Y., Coughlan, A. \& Wren, A. W. Investigating the surface reactivity of SiO2-TiO2$\mathrm{CaO}-\mathrm{Na} 2 \mathrm{O} / \mathrm{SrO}$ bioceramics as a function of structure and incubation time in simulated body fluid. J. Mater. Sci. Mater. Med. 25, 1853-1864 (2014). 
122. O’Donnell, M. D., Candarlioglu, P. L., Miller, C. A., Gentleman, E. \& Stevens, M. M. Materials characterisation and cytotoxic assessment of strontium-substituted bioactive glasses for bone regeneration. J. Mater. Chem. 20, 8934-8941 (2010).

123. Steinmann, P. A., Tardy, Y. \& Hintermann, H. E. Adhesion testing by the scratch test method: The influence of intrinsic and extrinsic parameters on the critical load. Thin Solid Films 154, 333-349 (1987).

124. De Bruyn, K., Celis, J. P., Roos, J. R., Stals, L. M. \& Van Stappen, M. Coating thickness and surface roughness of TiN-coated high speed steel in relation to coating functionality. Wear 166, 127-129 (1993).

125. Fukanuma H. \& Ohno N. Influence of Sunstrate Roughness and Temperature on Adhesive Strength in Thermal Spray Coatings.pdf. Therm. Spray (2003).

126. Wang, Y.-Y., Li, C.-J. \& Ohmori, A. Influence of substrate roughness on the bonding mechanisms of high velocity oxy-fuel sprayed coatings. Thin Solid Films 485, 141-147 (2005).

127. Della Bona, A., Borba, M., Benetti, P. \& Cecchetti, D. Effect of surface treatments on the bond strength of a zirconia-reinforced ceramic to composite resin. Braz. Oral Res. 21, 1015 (2007).

128. Matinmanesh, A. et al. Quantitative evaluation of the adhesion of bioactive glasses onto Ti6Al4V substrates. Mater. Des. 97, 213-221 (2016).

129. Ameli, A., Papini, M. \& Spelt, J. K. Evolution of crack path and fracture surface with degradation in rubber-toughened epoxy adhesive joints: Application to open-faced specimens. Int. J. Adhes. Adhes. 31, 530-540 (2011). 
130. ASTM International. ASTM D3433-99 Standard Test Method for Fracture Strength in Cleavage of Adhesives in Bonded Metal Joints. in (2012).

131. American Society of Mechanical Engineers. ASME - STANDARDS - Surface Texture (Surface Roughness, Waviness, and Lay). (ASME, 2009).

132. Yu, Y., Ashcroft, I. A. \& Swallowe, G. An experimental investigation of residual stresses in an epoxy-steel laminate. Int. J. Adhes. Adhes. 26, 511-519 (2006).

133. Donachie, M. J. Titanium: A Technical Guide, 2nd Edition. (ASM International, 2000).

134. JB Weld epoxies. Available at: www.JBweld.com.

135. Thompson, I. D. \& Hench, L. L. Mechanical properties of bioactive glasses, glass-ceramics and composites. Proc. Inst. Mech. Eng. [H] 212, 127-136 (1998).

136. Kokubo, T., Kim, H.-M. \& Kawashita, M. Novel bioactive materials with different mechanical properties. Biomaterials 24, 2161-2175 (2003).

137. Srivastava A. K., Pyare R. \& Singh S. P. Elastic Properties of substituted-45S5 Bioactive Glasses and Glass ceramics. Int. J. Sci. Eng. Res. 3, (2012).

138. Moore, D. R., Williams, J. G. \& Pavan, A. Fracture Mechanics Testing Methods for Polymers, Adhesives and Composites. (Elsevier, 2001).

139. Troczynski, T. \& Camire, J. On use of double cantilever beam for coatings and adhesion tests. Eng. Fract. Mech. 51, 327-332 (1995).

140. Wren, A. W. et al. Fabrication of $\mathrm{CaO}-\mathrm{NaO}-\mathrm{SiO} 2 / \mathrm{TiO} 2$ scaffolds for surgical applications. J. Mater. Sci. Mater. Med. 23, 2881-2891 (2012).

141. Hutchinson, J. W. \& Suo, Z. Mixed Mode Cracking in Layered Materials. in Advances in Applied Mechanics (ed. Wu, J. W. H. and T. Y.) 29, 63-191 (Elsevier, 1991). 
142. Nairn, J. A. Energy release rate analysis for adhesive and laminate double cantilever beam specimens emphasizing the effect of residual stresses. Int. J. Adhes. Adhes. 20, 59-70 (2000).

143. Howard, S. J. \& Clyne, T. W. Interfacial fracture toughness of vacuum-plasma-sprayed coatings. Surf. Coat. Technol. 45, 333-342 (1991).

144. Cotterell, B. \& Rice, J. R. Slightly curved or kinked cracks. Int. J. Fract. 16, 155-169 (1980).

145. Mirsayar, M. M. On fracture of kinked interface cracks - The role of T-stress. Mater. Des. 61, 117-123 (2014).

146. Mirsayar, M. M. \& Park, P. The role of T-stress on kinking angle of interface cracks. Mater. Des. 80, 12-19(2015).

147. Mirsayar, M. M. Mixed mode fracture analysis using extended maximum tangential strain criterion. Mater. Des. 86, 941-947 (2015).

148. Shlyannikov, V. N. T-stress for crack paths in test specimens subject to mixed mode loading. Eng. Fract. Mech. 108, 3-18 (2013).

149. Cornetti, P., Sapora, A. \& Carpinteri, A. T-stress effects on crack kinking in Finite Fracture Mechanics. Eng. Fract. Mech. 132, 169-176 (2014).

150. Ayatollahi, M. R. \& Saboori, B. T-stress effects in mixed mode I/II/III brittle fracture. Eng. Fract. Mech. 144, 32-45 (2015).

151. Gupta, M., Alderliesten, R. C. \& Benedictus, R. A review of T-stress and its effects in fracture mechanics. Eng. Fract. Mech. 134, 218-241 (2015). 
152. Rodriguez, O. et al. Silica-Based and Borate-Based, Titania-Containing Bioactive Coatings Characterization: Critical Strain Energy Release Rate, Residual Stresses, Hardness, and Thermal Expansion. J. Funct. Biomater. 7, 32 (2016).

153. Sola, A., Bellucci, D., Cannillo, V. \& Cattini, A. Bioactive glass coatings: a review. Surf. Eng. 27, 560-572 (2011).

154. Welsch, G., Boyer, R. \& Collings, E. W. Materials Properties Handbook: Titanium Alloys. (ASM International, 1993).

155. ASTM International. ASTM E228 Standard Test Method for Linear Thermal Expansion of Solid Materials with a Push-Rod Dilatometer. in (2016).

156. Hurt, J. C. \& Phillips, C. J. Structural Role of Zinc Oxide in Glasses in the System Na2OZnO-SiO2. J. Am. Ceram. Soc. 53, 269-273 (1970).

157. Elber, W. Effects of Shot-Peening Residual Stresses on the Fracture and Crack-Growth Properties of D6AC Steel. in Fracture Toughnessand Slow-Stable Cracking. Spec. Tech. Pub 45-58 (American Society of Testing and Materials, 1974). doi:10.1520/STP38591S

158. Sglavo, V. M., Larentis, L. \& Green, D. J. Flaw-Insensitive Ion-Exchanged Glass: I, Theoretical Aspects. J. Am. Ceram. Soc. 84, 1827-1831 (2001).

159. Bermejo, R. et al. Residual stresses, strength and toughness of laminates with different layer thickness ratios. Acta Mater. 54, 4745-4757 (2006).

160. Wiederhorn, S. M. Fracture Surface Energy of Glass. J. Am. Ceram. Soc. 52, 99-105 (1969).

161. Harding, D. S., Oliver, W. C. \& Pharr, G. M. Cracking During Nanoindentation and its Use in the Measurement of Fracture Toughness. MRS Online Proc. Libr. Arch. 356, (1994).

162. Venkateshan, S. P. Mechanical Measurements. (Ane Books, 2008). 
163. Clément, J. et al. Chemical Durability and Mechanical Properties of Calcium Phosphate Glasses with the Addition of Fe2O3, TiO2 and ZnO. Key Eng. Mater. 192-195, 621-624 (2001).

164. Rajendran, V., Gayathri Devi, A. V., Azooz, M. \& El-Batal, F. H. Physicochemical studies of phosphate based $\mathrm{P} 2 \mathrm{O} 5-\mathrm{Na} 2 \mathrm{O}-\mathrm{CaO}-\mathrm{TiO} 2$ glasses for biomedical applications. J. NonCryst. Solids 353, 77-84 (2007).

165. Yoshida, S., Tanaka, H., Hayashi, T., Matsuoka, J. \& Soga, N. Scratch Resistance of Sodium Borosilicate Glass. J. Ceram. Soc. Jpn. 109, 511-515 (2001).

166. Seal, A. K. et al. Effect of phase separation on the fracture toughness of SiO2-B2O3-Na2O glass. Bull. Mater. Sci. 28, 457-460 (2005).

167. Li, Y. et al. Characterization and fracture property of different strontium-containing boratebased glass coatings for Ti6Al4V substrates. J. Non-Cryst. Solids 458, 69-75 (2017).

168. Matinmanesh, A. et al. Quantifying the mode II critical strain energy release rate of borate bioactive glass coatings on Ti6Al4V substrates. J. Mech. Behav. Biomed. Mater. 75, 212221 (2017).

169. Fernlund, G. \& Spelt, J. K. Mixed-mode fracture characterization of adhesive joints. Compos. Sci. Technol. 50, 441-449 (1994).

170. Wang, H. \& Vu-Khanh, T. Use of end-loaded-split (ELS) test to study stable fracture behaviour of composites under mode II loading. Compos. Struct. 36, 71-79 (1996).

171. Blackman, B. R. K., Kinloch, A. J. \& Paraschi, M. The determination of the mode II adhesive fracture resistance, GIIC, of structural adhesive joints: an effective crack length approach. Eng. Fract. Mech. 72, 877-897 (2005). 
172. Barrett, J. D. \& Foschi, R. O. Mode II stress-intensity factors for cracked wood beams. Eng. Fract. Mech. 9, 371-378 (1977).

173. de Moura, M. F. S. F. \& de Morais, A. B. Equivalent crack based analyses of ENF and ELS tests. Eng. Fract. Mech. 75, 2584-2596 (2008).

174. Protter, M. H. \& Protter, P. E. Calculus with Analytic Geometry. (Jones \& Bartlett Learning, 1988).

175. Friedrich, K. Application of Fracture Mechanics to Composite Materials. (Elsevier, 2012).

176. Carlsson, L. A., Gillespie, J. W. \& Trethewey, B. R. Mode II Interlaminar Fracture of Graphite/Epoxy and Graphite/PEEK. J. Reinf. Plast. Compos. 5, 170-187 (1986).

177. O’Brien, T. K., Murri, G. B. \& Salpekar, S. A. Interlaminar Shear Fracture Toughness and Fatigue Thresholds for Composite Materias. ASTM Int. Composite materials: Fatigue and fracture, second volume, (1989).

178. Tanaka, K., Kageyama, K. \& Hojo, M. Prestandardization study on mode II interlaminar fracture toughness test for cfrp in japan. Composites 26, 257-267 (1995).

179. Evans, A. G., Dalgleish, B. J., He, M. \& Hutchinson, J. W. On crack path selection and the interface fracture energy in bimaterial systems. Acta Metall. 37, 3249-3254 (1989).

180. Evans, A. G. \& Hutchinson, J. W. Effects of non-planarity on the mixed mode fracture resistance of bimaterial interfaces. Acta Metall. 37, 909-916 (1989).

181. Cao, H. C., Thouless, M. D. \& Evans, A. G. Residual Stresses and Cracking in Brittle Solids Bonded with a Thin Ductile Layer. Acta Metall. 36, 2037-2046 (1988).

182. Gillanders, J. T., Riddle, R. A., Streit, R. D. \& Finnie, I. Methods for Determining the Mode I and Mode II Fracture Toughness of Glass Using Thermal Stresses. J. Eng. Mater. Technol. 112, 151-156 (1990). 
183. Watanabe, T., Muratsubaki, K., Benino, Y., Saitoh, H. \& Komatsu, T. Hardness and elastic properties of Bi2O3-based glasses. J. Mater. Sci. 36, 2427-2433 (2001).

184. Wang, W. H. Elastic moduli and behaviors of metallic glasses. J. Non-Cryst. Solids 351, 1481-1485 (2005).

185. Shinkai, N., Bradt, R. C. \& Rindone, G. E. Elastic Modulus and Fracture Toughness of Ternary PbO-ZnO-B2O3 Glasses. J. Am. Ceram. Soc. 65, 123-126 (1982).

186. Fleck, N. A., Hutchinson, J. W. \& Zhigang, S. Crack path selection in a brittle adhesive layer. Int. J. Solids Struct. 27, 1683-1703 (1991).

187. Silva, L. F. M. da, Dillard, D. A., Blackman, B. \& Adams, R. D. Testing Adhesive Joints: Best Practices. (John Wiley \& Sons, 2012).

188. Matinmanesh, A. et al. Evaluating the critical strain energy release rate of bioactive glass coatings on Ti6Al4V substrates after degradation (Accepted). J. Mech. Behav. Biomed. Mater. (2017). doi:10.1016/j.jmbbm.2017.11.015

189. Li, Y. et al. Investigating the influence of $\mathrm{Na}+$ and $\mathrm{Sr} 2+$ on the structure and solubility of SiO2-TiO2-CaO-Na2O/SrO bioactive glass. J. Mater. Sci. Mater. Med. 26, 85 (2015).

190. Tilocca, A. \& Cormack, A. N. Surface Signatures of Bioactivity: MD Simulations of 45S and 65S Silicate Glasses. Langmuir 26, 545-551 (2010).

191. Tilocca, A. \& Cormack, A. N. Modeling the Water-Bioglass Interface by Ab Initio Molecular Dynamics Simulations. ACS Appl. Mater. Interfaces 1, 1324-1333 (2009).

192. Jones, J. R. Review of bioactive glass: From Hench to hybrids. Acta Biomater. 9, 44574486 (2013).

193. Sodium migration pathways in multicomponent silicate glasses: Car-Parrinello molecular dynamics simulations. J. Chem. Phys. 133, 014701 (2010). 
194. Lao, J., Nedelec, J. M. \& Jallot, E. New strontium-based bioactive glasses: physicochemical reactivity and delivering capability of biologically active dissolution products. J. Mater. Chem. 19, 2940-2949 (2009).

195. Han, Y., Xu, K. \& Lu, J. Dissolution response of hydroxyapatite coatings to residual stresses. J. Biomed. Mater. Res. 55, 596-602 (2001).

196. Ellis, R. J. Macromolecular crowding: obvious but underappreciated. Trends Biochem. Sci. 26, 597-604 (2001).

197. Nychka, J. A., Mazur, S. L. R., Kashyap, S., Li, D. \& Yang, F. Dissolution of bioactive glasses: The effects of crystallinity coupled with stress. JOM 61, 45-51 (2009).

198. Li, D., Yang, F. \& Nychka, J. Indentation-induced residual stresses in 45S5 bioglass and the stress effect on the material dissolution. Eng. Fract. Mech. 75, 4898-4908 (2008).

199. Zhang, K., Yan, H., Bell, D. C., Stein, A. \& Francis, L. F. Effects of materials parameters on mineralization and degradation of sol-gel bioactive glasses with 3D-ordered macroporous structures. J. Biomed. Mater. Res. A 66, 860-869 (2003).

200. Hench, L. L. \& Polak, J. M. Third-generation biomedical materials. Science 295, 10141017 (2002).

201. Ameli, A., Azari, S., Papini, M. \& Spelt, J. K. Crack path selection in the fracture of fresh and degraded epoxy adhesive joints. Eng. Fract. Mech. 78, 1986-2003 (2011).

202. Azari, S., Papini, M. \& Spelt, J. K. Effect of Surface Roughness on the Performance of Adhesive Joints Under Static and Cyclic Loading. J. Adhes. 86, 742-764 (2010).

203. Prolongo, S. G., Rosario, G. \& Ureña, A. Study of the effect of substrate roughness on adhesive joints by SEM image analysis. J. Adhes. Sci. Technol. 20, 457-470 (2006). 